\title{
Mycobacterium tuberculosis and the human macrophage - shifting the balance through inflammasome activation
}

\section{Daniel Eklund}

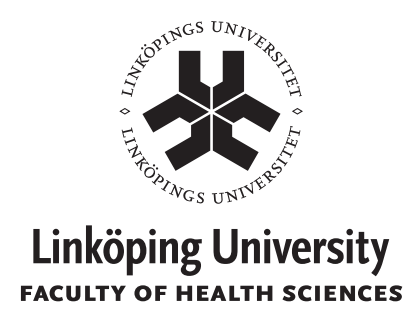

Division of Medical Microbiology

Department of Clinical and Experimental Medicine

Faculty of Health Sciences

Linköping University

SE-58185 Linköping, Sweden 
Copyright (C) Daniel Eklund, 2013

All rights reserved.

Paper I is reprinted with permission from the American Society for Microbiology, Paper III is reprinted with permission from Elsevier (published in the International Journal of Mycobacteriology) and Paper V is reprinted with permission from Oxford Journals (published in the Journal of Infectious Diseases).

ISBN: 978-91-7519-558-2

ISSN: 0345-0082

Printed by LiU-Tryck, Linköping, Sweden 2013 
"Why does it not work, Daniel?!!"

- Former project student Atemnkeng Ambrose, when theory and practice fails to come together 


\section{Supervisor}

Maria Lerm, Linköping University, Sweden

\section{Co-supervisors}

Olle Stendahl, Linköping University, Sweden

Eva Särndahl, Örebro University, Sweden

\section{Faculty opponent}

Norbert Reiling, Research Center Borstel, Germany

\section{Funding}

This work was supported by the Swedish Research Council, the Bill \& Melinda Gates Foundation, the Ekhaga Foundation, the Carl Trygger Foundation, the Swedish Heart Lung Foundation, SIDA/SAREC, the County Council of Östergötland, the Research Council of Southeast Sweden (FORSS), King Gustaf V 80-Year Memorial Foundation, Oskar II Jubilee Foundation, Clas Groschinsky Foundation, the Söderbergs Foundation and the Swedish Society of Medicine. 


\section{TABLE OF CONTENTS}

ABSTRACT

POPULÄRVETENSKAPLIG SAMMANFATTNING ....................................... 3

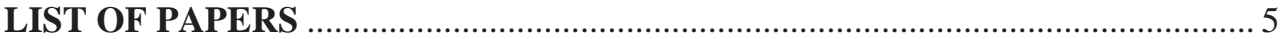

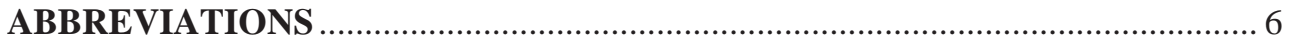

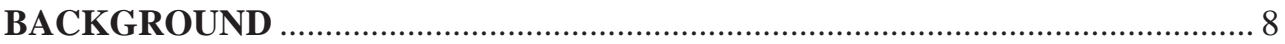

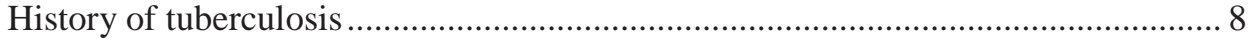

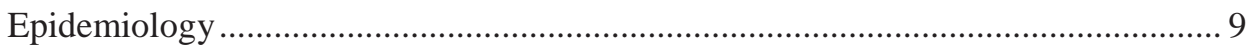

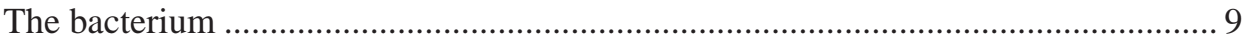

Evolution of Mycobacterium tuberculosis ...................................................... 10

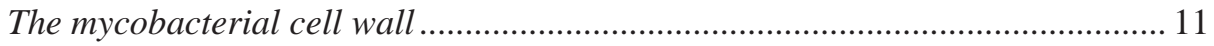

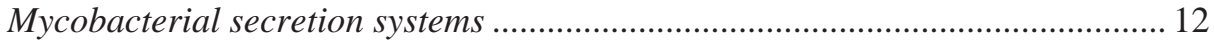

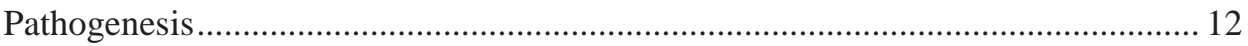

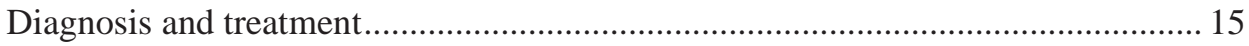

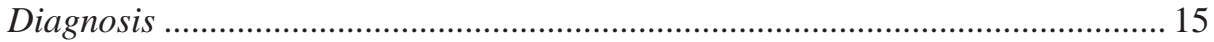

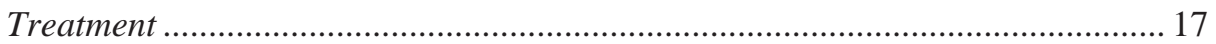

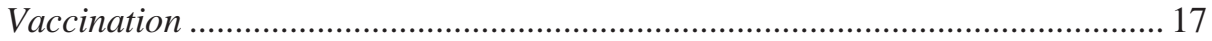

Pathogen recognition in tuberculosis ............................................................. 18

C-type lectins and complement receptor 3 ..................................................... 18

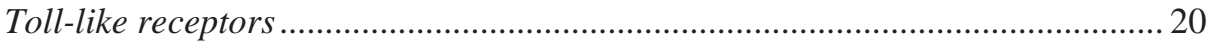

Nucleotide-binding oligomerization domain-like receptors ............................... 21

Macrophage effector functions and Mycobacterium tuberculosis.......................... 24

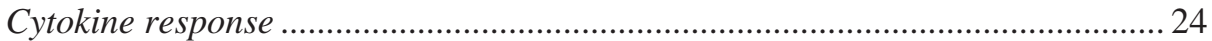

Macrophage activation and polarization ....................................................... 26

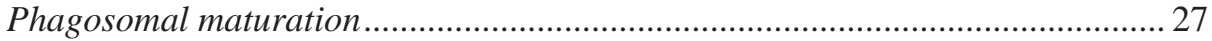

The fate of the mycobacterial phagosome ......................................................... 30

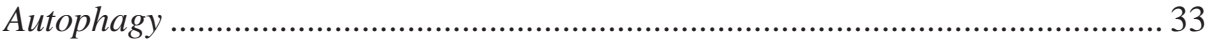

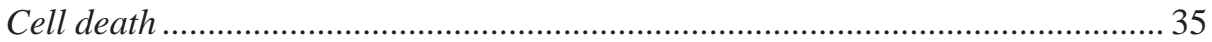

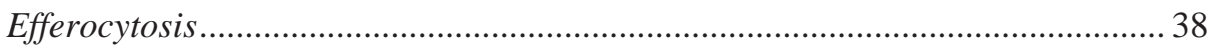

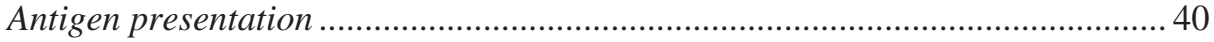




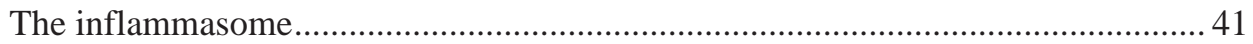

Structure of the inflammasome .................................................................... 41

Activation of the inflammasome ...................................................................... 42

Genetic alterations of the inflammasome ......................................................... 46

Regulation of the inflammasome and $I L-1 \beta$ production .................................... 47

Inflammasome-related cell death ..................................................................... 49

Non-canonical functions of the inflammasome .................................................. 51

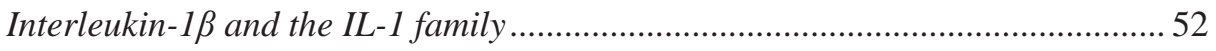

Interleukin-1 $\beta$ and inflammasome activation in tuberculosis ............................ 54

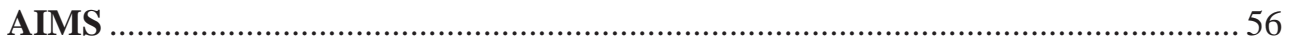

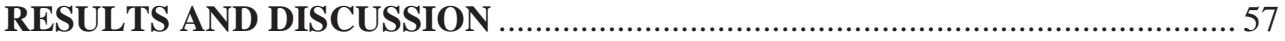

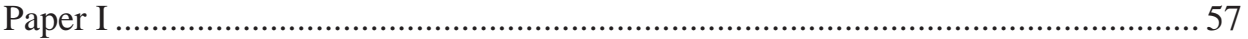

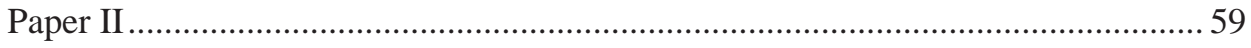

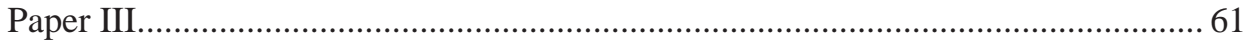

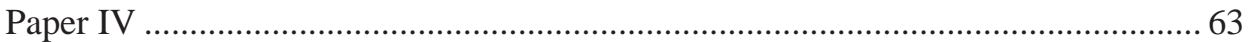

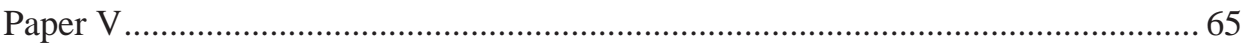

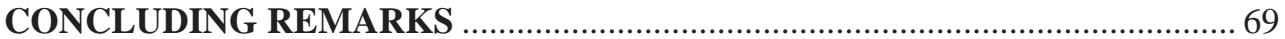

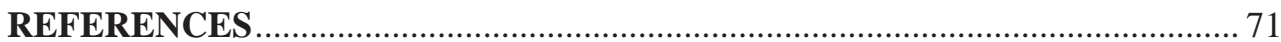

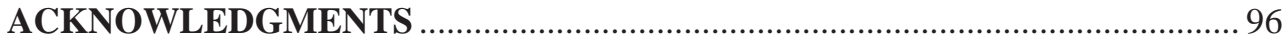




\section{ABSTRACT}

Mycobacterium tuberculosis is a very successful pathogen and tuberculosis constitutes a major threat to global health worldwide. The World Health Organization (WHO) estimates that almost nine million new cases and 1.5 million deaths occur annually and the situation is worsened by increased antibiotic resistance and an extreme synergism with the HIV pandemic. M. tuberculosis primarily affects the lungs where the infection can lead to either eradication of the bacteria or the initiation of an immune response that culminates in the formation of a large cluster of immune cells termed granulomas. In these granulomas, the bacteria can either replicate and cause disease with the ultimate goal of spreading to new hosts or cause latent tuberculosis, which can persist for decades. The tools available to manage the disease are currently suboptimal and include lengthy antibiotic treatments and an inefficient vaccine resulting in poor protection. On a cellular level, M. tuberculosis primarily infects the cell designed to recognize, ingest and eradicate bacteria, namely the human macrophage. Following recognition, the macrophage phagocytoses the bacterium and tries to kill it using an array of different effector mechanisms including acidification of the bacteriumcontaining vacuole, different degradative enzymes and the generation of radicals. However, the bacterium is able to circumvent many of these harmful effects, leading to a tug-of-war between the bacterium and host macrophage. This thesis aims at studying the interaction between the human macrophage and $M$. tuberculosis to identify host factors critical for controlling growth of the bacteria. More specifically, it focuses on the role of an intracellular receptor protein called NLRP3 and its downstream effects. NLRP3 is activated in human macrophages infected by $M$. tuberculosis and upon activation it forms a multi-protein complex known as the inflammasome. This protein complex can induce the production of the proinflammatory cytokine IL-1 $\beta$ and specialized forms of macrophage cell death. We hypothesized that stimulating this pathway would have a beneficial effect for the host macrophage during infection with $M$. tuberculosis.

To allow us to follow interaction between $M$. tuberculosis and the human macrophage, we first developed a luminometry-based method of measuring bacterial numbers and following bacterial growth over several days in infected cells. With this new assay we showed that low numbers of bacteria induced very low levels of IL-1 $\beta$ and failed to induce any type of cell death in the macrophage. However, when a critical number of bacteria were reached, the infected macrophages underwent necrosis, which was accompanied by high levels of IL-1 $\beta$. We were also able to show that addition of vitamin $\mathrm{D}$, which has been implicated as an important factor for increased killing capacity of infected macrophages, increased the production of IL-1 $\beta$, which coincided 
with increased killing of $M$. tuberculosis. This effect was seen specifically in cells from patients with active tuberculosis, suggesting that these cells are primed to respond to vitamin $\mathrm{D}$ and increased levels of IL-1 $\beta$. Furthermore, we also showed that increasing production of IL-1 $\beta$ by stimulating infected macrophages with apoptotic neutrophils in turn drives the production of other proinflammatory cytokines. Lastly, we showed that gain-of-function polymorphisms in inflammasome components linked to increased inflammasome activation and IL-1 $\beta$ production promotes bacterial killing in human macrophages. In conclusion, the work presented in this thesis shows that by enhancing the functions of the inflammasome, it is possible to tip the balance between the human macrophage and $M$. tuberculosis in favor of the host cell. 


\section{POPULÄRVETENSKAPLI G SAMMANFATTNI NG}

Tuberkulos, en av världens vanligaste infektionssjukdomar, orsakas av bakterien Mycobacterium tuberculosis och nästan 1.5 miljoner människor dör årligen av sjukdomen. Läget förvärras ytterligare av att antibiotikaresistensen ökar och det faktum att bakterien har lättare att orsaka sjukdom hos HIV-positiva personer. Tuberkulos smittar främst genom att man andas in droppar som innehåller bakterier som sjuka individer hostar upp. Dessa droppar hamnar sedan i lungan där bakterien äts upp av en typ av vit blodkropp s.k. makrofager eller "storätarceller". Detta resulterar i vissa fall i att makrofagen lyckas döda bakterien, men många gånger lyckas bakterien överleva i den vita blodkroppen. Makrofagen utsöndrar då istället vissa signalämnen som drar till sig fler vita blodkroppar. Alla dessa vita blodkroppar bildar tillsammans en speciell struktur, s.k. granulom, som omsluter den infekterade cellen och försöker hindra bakterien från att sprida sig i kroppen. Bakterien kan nu antingen gå in i en slags dvala och vänta på bättre förutsättningar eller försöka ta sig ut från granulomet och sprida sig till nya individer. Det är när bakterien tar sig ut från granulomet som sjukdom faktiskt uppstår och individen börjar hosta droppar med bakterier som kan ta sig ner i lungorna hos nästa individ. För att motverka spridning av sjukdomen och försöka bota infektionen så finns både ett vaccin och antibiotikabehandling tillgänglig, men tyvärr har vaccinet visats sig ge dåligt skydd och antibiotikabehandlingen är väldigt lång med mycket biverkningar. Även själva diagnosticeringen av sjukdomen är svår och det tar lång tid innan man vet om patienten faktiskt lider av tuberkulos.

En väldigt viktig faktor vid tuberkulos är samspelet mellan bakterien och makrofagen i lungan efter det att makrofagen har ätit bakterien. Denna avhandling undersöker samspelet mellan denna vita blodkropp och $M$. tuberculosis för att identifiera vilka faktorer som är viktiga för att förstärka makrofagens avdödningsförmåga. Avhandlingen fokuserar främst på en speciell molekyl inuti makrofagen som kallas NLRP3 och som aktiveras när makrofagen äter upp M. tuberculosis. Aktivering av NLRP3 leder i sin tur till utsöndring av ett signalämne som kan förstärka makrofagens avdödningsförmåga. Detta signalämne kallas IL-1 $\beta$. För att studera samspelet mellan vita blodkroppar och bakterier, utvecklade vi först en metod för att mäta mängden bakterier inuti makrofager. Med denna metod har vi visat att bakterien inte aktiverar utsöndringen av signalämnet IL-1 $\beta$ förrän de blir tillräcklig många inuti makrofagen. När detta sker lyckas bakterierna döda cellerna de lever i genom en form av celldöd som kallas nekros och detta sker samtidigt som IL-1 $\beta$ utsöndras. Vi har också visat att genom tillsats av D-vitamin, som också fungerar som ett signalämne som förstärker 
makrofagens funktioner, till infekterade celler kan man öka mängden utsöndrat IL-1 $\beta$. D-vitamin förstärker samtidigt avdödningsförmågan hos makrofager från patienter som lider av tuberkulos men inte från individer utan tuberkulos. Vidare visar vi att andra sätt att stimulera NLRP3 och öka utsöndringen av IL-1 $\beta$ har positiva effekter på makrofagens funktioner. Stimulerar man infekterade makrofager med en annan typ av vita blodkroppar som redan är döda, så utsöndrar makrofagen ökade mängder av flera andra signalämnen som kan hjälpa till att öka avdödningsförmågan. Slutligen visar vi att vissa förändringar i arvsmassan som leder till att NLRP3-molekylen är konstant aktiverad ökar avdödningsförmågan hos infekterade makrofager. Tillsammans visar dessa studier hur man genom att stimulera ökad aktivitet hos NLRP3 och högre utsöndring av IL-1 $\beta$ kan få makrofager att bättre avdöda $M$. tuberculosis. Denna kunskap kan bidra till utvecklingen av nya läkemedel som specifikt kan stimulera makrofager till bättre avdödning samt en större förståelse vad som avgör om man blir sjuk eller inte när man utsätts för bakterien. 


\section{ST OF PAPERS}

\section{Paper I}

Eklund D*, Welin A*, Schön T, Stendahl O, Huygen K \& Lerm M. Validation of a medium-throughput method for evaluation of intracellular growth of Mycobacterium tuberculosis. Clinical and Vaccine Immunology. 17(4): 513-17, 2010.

*These authors contributed equally

\section{Paper II}

Welin A*, Eklund D*, Stendahl O \& Lerm M. Human macrophages infected with a high burden of ESAT-6-expressing $M$. tuberculosis undergo caspase-1- and cathepsin B-independent necrosis. PLoS One. 2011;6(5).

*These authors contributed equally

\section{Paper III}

Eklund D, Persson HL, Larsson M, Welin A, Idh J, Paues J, Fransson S, Stendahl O, Schön T \& Lerm M. Vitamin D enhances IL-1及 secretion and restricts growth of Mycobacterium tuberculosis in macrophages from TB patients. International Journal of Mycobacteriology. 2(1): 18-25, 2012.

\section{Paper IV}

Andersson H, Eklund D, Ngoh E, Persson A, Andersson B, Svensson K, Lerm M \& Stendahl O. Apoptotic neutrophils augment the inflammatory response to Mycobacterium tuberculosis infection in human macrophages. Manuscript.

\section{Paper V}

Eklund D, Welin A, Andersson H, Verma D, Söderkvist P, Stendahl O, Särndahl E \& Lerm M. Human gene variants linked to enhanced NLRP3 activity limit intramacrophage growth of Mycobacterium tuberculosis. Journal of Infectious Diseases. Epub ahead of print, Oct 24, 2013. 


\section{ABBREVI ATI ONS}

\begin{tabular}{|c|c|}
\hline AIM2 & absent in melanoma 2 protein \\
\hline $\mathrm{AM}$ & acetoxymethyl \\
\hline APC & antigen-presenting cell \\
\hline ASC & apoptosis-associated speck-like protein containing a CARD \\
\hline Atg & autophagy-related proteins \\
\hline BCG & Bacillus Calmette-Guérin \\
\hline CAPS & cryopyrin-associated periodic syndromes \\
\hline CARD & caspase activation and recruitment domain \\
\hline CARD8 & caspase recruitment domain family, member 8 \\
\hline CFP-10 & $10 \mathrm{kDa}$ culture filtrate protein \\
\hline CIITA & class II transactivator \\
\hline COP & CARD-only protein \\
\hline CR3 & complement receptor 3 \\
\hline DAMP & damage/danger-associated molecular pattern \\
\hline DC & dendritic cell \\
\hline DC-SIGN & $\begin{array}{l}\text { dendritic cell-specific intercellular adhesion molecule-3- } \\
\text { grabbing non-integrin }\end{array}$ \\
\hline DISC & death-inducing signaling complex \\
\hline DOTS & directly observed treatment, short-course program \\
\hline EEA1 & early endosomal antigen 1 \\
\hline ER & endoplasmic reticulum \\
\hline ESAT-6 & 6-kDa early secreted antigenic target \\
\hline HMGB1 & high-mobility group protein 1 \\
\hline HSP90 & heat-shock protein 90 \\
\hline IFN- & interferon- \\
\hline IGRA & IFN- $\gamma$ release assay \\
\hline IL- & interleukin- \\
\hline IL-1Ra & interleukin-1 receptor antagonist \\
\hline iNOS & inducible nitric oxide synthase \\
\hline IRAK & interleukin-1 receptor-associated kinase \\
\hline LAM & lipoarabinomannan \\
\hline LAMP & lysosomal-associated membrane protein \\
\hline LC3 & microtubule-associated protein 1A/1B-light chain 3 \\
\hline LM & lipomannan \\
\hline LPS & lipopolysaccharide \\
\hline LRR & leucine-rich repeats \\
\hline $\mathrm{LT}$ & anthrax lethal toxin \\
\hline
\end{tabular}




\begin{tabular}{|c|c|}
\hline $\mathrm{LXA}_{4}$ & lipoxin $\mathrm{A}_{4}$ \\
\hline ManLAM & mannose-capped lipoarabinomannan \\
\hline MDP & muramyl dipeptide \\
\hline MDR/XDR/TDR & multi-, extensively- or totally drug resistant \\
\hline MHC & major histocompatibility complex \\
\hline MOI & multiplicity of infection \\
\hline MR & mannose receptor \\
\hline MTBC & Mycobacterium tuberculosis complex \\
\hline mTOR & mammalian target of rapamycin \\
\hline$N F-\kappa B$ & nuclear factor kappa B \\
\hline NLR & $\begin{array}{l}\text { nucleotide-binding oligomerization domain (NOD)- } \\
\text { like receptors }\end{array}$ \\
\hline NLRC & NOD-like receptors subfamily C (containing CARD) \\
\hline NLRP & NOD-like receptors subfamily P (containing PYD) \\
\hline NO & nitric oxide \\
\hline Nod & nucleotide-binding oligomerization domain \\
\hline NOX2 & phagocytic NADPH oxidase \\
\hline NRAMP1 & natural resistance-associated macrophage protein 1 \\
\hline PAMP & pathogen-associated molecular pattern \\
\hline $\mathrm{PGE}_{2}$ & prostaglandin $\mathrm{E}_{2}$ \\
\hline PILAM & phosphoinositol-capped LAM \\
\hline PIM & phosphatidylinositol mannosides \\
\hline POP & Pyrin-only protein \\
\hline PPD & purified protein derivative \\
\hline PRR & pathogen recognition receptor \\
\hline PYD & Pyrin domain \\
\hline RIP & receptor-interacting serine/threonine-protein kinase \\
\hline RNS & reactive nitrogen species \\
\hline ROS & reactive oxygen species \\
\hline SGT1 & suppressor of G2 allele of skp1 \\
\hline SLR & sequestosome $1 / p 62$-like receptor \\
\hline SNARE & $\begin{array}{l}\text { soluble } \mathrm{N} \text {-ethylmaleimide-sensitive factor activating protein } \\
\text { receptor protein }\end{array}$ \\
\hline TIR & Toll/interleukin-1 receptor domain \\
\hline TLR & Toll-like receptor \\
\hline TNF & tumor necrosis factor \\
\hline TST & tuberculin skin test \\
\hline TXNIP & thioredoxin (TRX)-interacting protein \\
\hline VDR & vitamin D receptor \\
\hline WHO & World Health Organization \\
\hline
\end{tabular}




\section{BACKGROUND}

\section{History of tuberculosis}

Tuberculosis is a disease caused by the bacterium Mycobacterium tuberculosis. It is one of the oldest known infectious diseases that has plagued mankind since the expansion of the modern human out of Africa (1-2), and archaeological findings have shown the presence of DNA from the bacterium in 1000-year old mummies from different places around the world (3-4). Records of tuberculosis date back to ancient times and the disease has been referred to as phthisis, consumption and "the white plague". During the $17^{\text {th }}, 18^{\text {th }}$ and $19^{\text {th }}$ century, the disease reached epidemic proportions due to increased urbanization, where factors such as crowded living conditions and malnutrition fueled the spread of the bacterium and in the beginning of the $19^{\text {th }}$ century, death-rates due to tuberculosis in Stockholm were approaching 1000 deaths per 100000 inhabitants/year (5).

The cause of the disease was unknown up until the $24^{\text {th }}$ of March 1882 when the German physician Robert Koch announced that he had managed to isolate the causative bacterium $M$. tuberculosis (6), a feat for which he later received the Nobel Prize. The discovery led to renewed efforts to find treatments against the disease, but it was not until 1943 when another Nobel Prize laureate, Selman A. Waksman, discovered streptomycin that tuberculosis could be treated for the first time (7). Over the following 14 years, most antimycobacterial compounds currently in use today were discovered, including para-amino salicylic acid (PAS), which was first described by the Swedish physician and chemist Jörgen Lehmann (5). However, due to improved living conditions and to some extent the practice of sending tuberculosis patients to secluded and specialized nursing homes called sanatoria, a decline in mortality rates by tuberculosis was seen long before the introduction of antibiotics. Though this decline has continued to present time as living conditions have continued to improve, reduced mortality has mainly been confined to the Western world (5). Today, tuberculosis still remains a large threat against public health on a global scale and despite being an ancient disease, new challenges in the shape of antibiotic resistance and co-infection by HIV shows that this infectious disease is far from extinct. 


\section{Epidemiology}

WHO refers to the global burden of tuberculosis as still being enormous with 8.7 million new cases and 1.4 million deaths in 2011, where one third of the fatalities were in HIV-positive individuals. Though incidence rates are slowly declining globally they remain high among low-income countries in Asia and Africa (Figure 1). In addition, the countries with the highest incidence of tuberculosis also show the highest prevalence of HIV-positive tuberculosis patients (e.g. Sub-Saharan Africa), underlining the synergistic effect between tuberculosis and HIV (8). On a global scale, $3-7 \%$ of all new tuberculosis patients and $20 \%$ of previously treated patients are thought to be infected with multi-resistant tuberculosis (MDR), meaning that the causative strain is resistant to the two first-line drugs isoniazid and rifampicin. Furthermore, 84 countries have to date reported at least one case of extensively drug resistant tuberculosis (XDR) which is defined as tuberculosis caused by strains resistant to the first-line drugs rifampicin and isoniazid as well as to fluoroquinolones and any of the second-line drugs such as amikacin, kanamycin and capreomycin (9). In 2009, the first cases of totally drug resistant tuberculosis (TDR) were reported, where the strains tested were resistant to all tested first-line and second-line drugs (10). In Sweden, 645 cases of tuberculosis were reported in 2012, where 12 cases were identified as MDR and 2 cases as XDR. A large majority of the patients were foreignborn residents from countries where tuberculosis is endemic (11). In summary, though WHO's 2015 Millennium Development Goal of globally halting and reversing incidence of tuberculosis has been achieved, the 2050 Millennium Development Goal of eliminating tuberculosis is not going to be achieved according to the current prognosis (8).

\section{The bacterium}

M. tuberculosis is an intracellular bacterium whose preferred host cell is the human macrophage. It is a slow-growing and rod-shaped bacterium with a generation time of about 24 hours. It is classified as a Gram-positive bacterium, but has a cell wall with an additional outer layer of unusual lipids, mainly mycolic acid. This feature has been used to identify mycobacteria in the laboratory for over a century by using staining techniques referred to as acid-fast or Ziehl-Neelsen staining, first developed by Franz Ziehl and Friedrich Neelsen (12). 


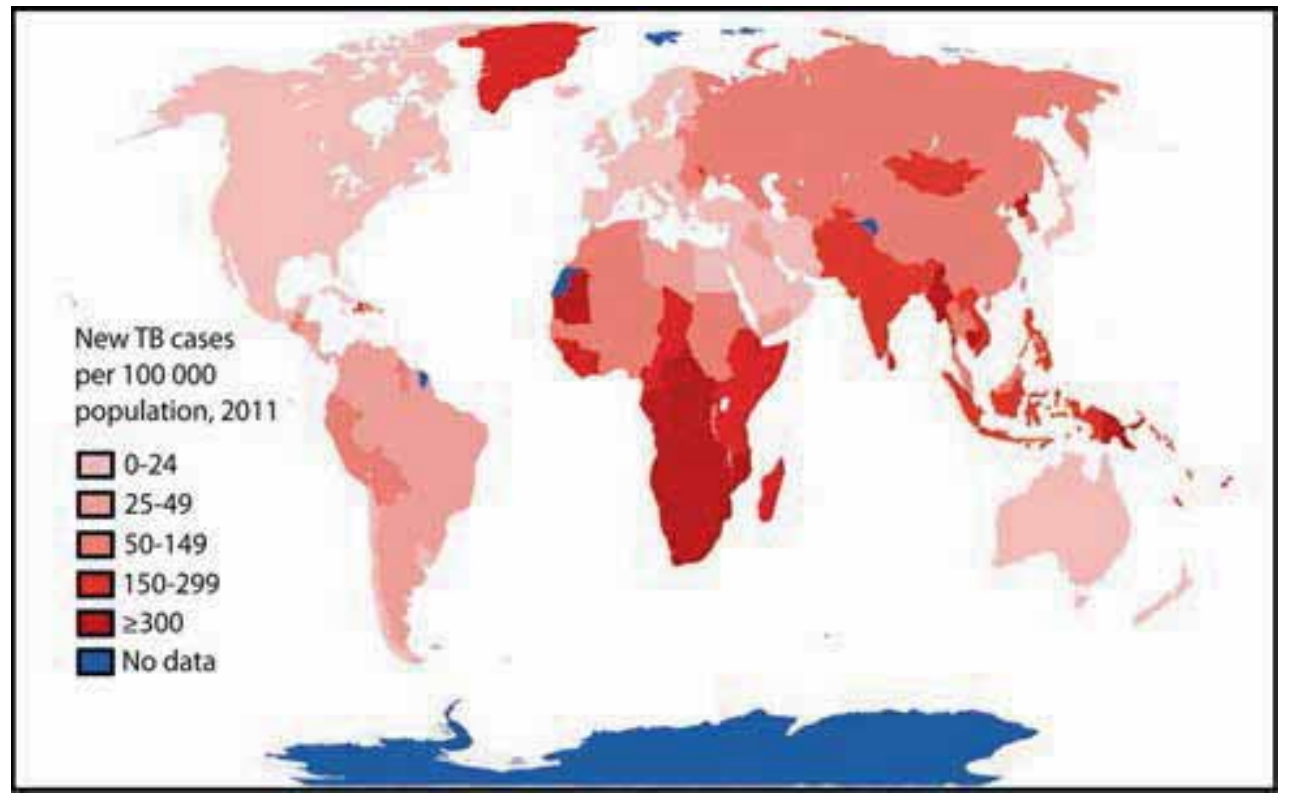

Figure 1. The global incidence of all forms of tuberculosis by country/region, 2011.

\section{Evolution of Mycobacterium tuberculosis}

M. tuberculosis is part of a phylogenetically closely related complex termed $M$. tuberculosis complex (MTBC) of several mycobacterial species, that all cause tuberculosis in their respective host species (13-14). The similarity between members of the MTBC strongly suggests a common ancestor that over time has been transferred and adapted to different hosts. It was earlier thought that M. tuberculosis arose from $M$. bovis, which causes tuberculosis in cattle, around the time when man first domesticated cattle roughly 10000 years ago. However, comparative genome analyses have shown that $M$. tuberculosis is unlikely to have been derived from its bovine counterpart, and the more likely scenario is that tuberculosis was actually transferred from man to animal (15). M. tuberculosis can be further subdivided into several strain lineages that are genetically distinct from each other. In fact, the average genetic distance between two human-adapted strains is equal to the average genetic distance between the animal-adapted strains, even though the latter have adapted to different host species. This diversity within M. tuberculosis can be tightly linked to migration events and demographic changes in human history, resulting in the fact that certain strain lineages of $M$. tuberculosis are overrepresented in specific parts of the world (16) and phylogeographic distribution of different strains suggest that they are particularly adapted to infect their sympatric human hosts (17). So far, seven major strain lineages of $M$. tuberculosis have been identified (18) and they can be roughly 
divided into ancient lineages and modern lineages, the former of which are genetically closer to a common ancestor than the modern ones. The modern lineages induce weaker inflammatory responses early in the disease, which might lead to faster progression and transmission, beneficial in modern times, where human populations are denser. The ancient lineages probably benefitted from staying latent until being able to infect a new generation of hosts within small human populations (19). Finally, a striking difference between $M$. tuberculosis and many other pathogens is that the most evolutionary conserved parts of the genome are in fact epitopes recognized by human T cells, implying that $M$. tuberculosis actually benefits from recognition by the human immune system (20). Taken together, the adaption of strains to particular groups of humans and to changes in the density of human populations indicates a close evolutionary relationship between host and pathogen.

\section{The mycobacterial cell wall}

Many of the characteristics of M. tuberculosis, as well as many of its virulent traits can be attributed to its unique cell wall composition. The mycobacterial cell wall consists of an outer segment and inner segment. The outer segment is mainly composed of free lipids, while the inner segment consists of peptidoglycan covalently linked to arabinogalactan, which in turn is attached to mycolic acid. This inner segment or cell wall core is referred to as the mycolyl-arabinogalactan-peptidoglycan complex (21). Throughout the cell wall, both cell wall proteins and lipoglycans can be found. These lipoglycans include phosphatidylinositol mannosides (PIMs) that upon additional glycosylation steps can form lipomannan (LM) and lipoarabinomannan (LAM). The PIMs, LM and LAM all attach non-covalently to the plasma membrane by their phosphatidyl-myo-inositol anchor from where they extend outwards (22). Though not being the only bioactive lipids present in the cell wall, PIM, LM and especially LAM has received a lot of attention due to their ability to modulate the host immune response. LAM can be further modified by addition of either mannose (ManLAM) or phosphoinositol (PILAM) caps, where the former are mainly found in pathogenic mycobacteria such as $M$. tuberculosis, while the latter are more associated with nonpathogenic mycobacterial species. PILAM as well as the precursor LM can induce production of cytokines (interleukin (IL)-12, IL-8 and tumor necrosis factor (TNF)) as well as to induce apoptosis in macrophages, while ManLAM from pathogenic bacteria fails to do so (23-24). In fact, ManLAM from M. tuberculosis actively inhibit many important antimicrobial mechanisms in macrophages (25-27). Certain cell wall proteins, such as the $19 \mathrm{kDa}$ lipoprotein, also act in a similar fashion, modulating the immune response of the host (28). 


\section{Mycobacterial secretion systems}

Mycobacteria do not only interact and modulate the surrounding environment through their cell wall components, but also by actively secreting proteins. Due to the rather complex mycobacterial cell wall, the majority of protein secretion in mycobacteria is achieved through a set of secretion systems unique to mycobacteria, termed type VII secretion systems or ESX systems. M. tuberculosis has five of these ESX systems, which are numbered ESX-1 through ESX-5 (29). Studies have shown that all of these systems arose through gene duplication of ESX-4, the only system that has orthologs in other types of bacteria (30). The typical substrates for type VII secretion systems are so called Esx proteins, which are secreted as dimeric complexes together with another type of Esx protein (31). However, other types of proteins, such as members of the PE and PPE proteins family can also be secreted through ESX systems (32). Genetic screens have shown that ESX-4 and ESX-2 are non-essential systems (33) and so far, no known function has been identified for either system. ESX-3, on the other hand, is essential for $M$. tuberculosis and is known to secrete the heterodimeric complex EsxG/EsxH (31). ESX-3 and its substrates are thought to be involved in homeostasis of metals, such as zinc and iron (34). The most intensively studied ESX systems are the two remaining members, ESX-1 and ESX-5. A number of substrates have been identified for ESX-1, the most well studied being the 6-kDa early secreted antigenic target (ESAT-6) and $10 \mathrm{kDa}$ culture filtrate protein (CFP-10) complex (35-36). The ESX-1 system is crucial for the early stages of infection by promoting both intracellular growth and cytolysis of macrophages. Deletion of ESX-1 and loss of subsequent protein secretion results in loss of virulence in M. tuberculosis (37-38). Avirulent strains of mycobacteria, such as the vaccine strain BCG or the commonly used lab strain H37Ra, are also characterized by deletion of the ESX-1 system and failure to secrete ESAT-6 (39-40). The ESX-5 system has a number of known substrates, including several PE and PPE proteins (41), perhaps making it the most versatile of the ESX systems. Mutants lacking ESX-5 show mixed effects on cytokine production in macrophages, displaying both proinflammatory and anti-inflammatory properties, while the ability to grow intracellularly remains unaffected. This together with the fact that ESX-5 is dependent on the preceding actions of ESX-1 has led to the idea that ESX-5 is important at later stages of macrophage infection and perhaps at early stages of granuloma formation (42-44).

\section{Pathogenesis}

Several organs can be affected by tuberculosis but it is mainly a pulmonary disease. Bacteria are transmitted between hosts through small aerosols, which are formed 
during coughing by patients with active tuberculosis. When inhaled by another individual, the bacterium will make its way into the lungs, where it will be recognized by resident alveolar macrophages. Following uptake, several scenarios are possible, where the outcome is dependent on multiple factors such as bacterial virulence and immune status or genetic determinants of the host. Bacteria can be eliminated, establish a characteristic latent infection, or the infection can progress to active tuberculosis. The risk of developing clinical tuberculosis after inhalation of $M$. tuberculosis is rather small and over $90 \%$ of those exposed either eliminate the bacteria, sometimes without developing adaptive immune responses, or become latently infected. Those who become latently infected have a $10 \%$ life-time risk to develop reactivation tuberculosis. Immune impairment, such as HIV co-infection, increases the risk considerably (45-46).

After M. tuberculosis is recognized and taken up by the alveolar macrophage, the bacterium interferes with key antimicrobial mechanisms and can start replicating intracellularly. If the infected cell is unable to handle the infection it will produce a range of cytokines and chemokines that will attract additional monocytes, neutrophils and dendritic cells (DCs). Traditionally, this stage of infection is thought to promote bacterial growth, and it is not until adaptive immunity is initiated that bacterial numbers stabilize due to the strong macrophage activation of interferon (IFN)- $\gamma$ produced by CD4+ cells arriving at the scene of infection (47). However, in recent years, several IFN- $\gamma$-independent macrophage stimulation pathways and mechanisms have also been shown to induce intracellular killing in human macrophages, suggesting that the battle between host and pathogen can be decided before involvement of adaptive immunity (48-50). After arriving at the scene, immune cells of both the innate and adaptive immunity form the classical granuloma (Figure 2); a macroscopic structure with heavily infected macrophages surrounded by multinucleate (giant cells) and lipid-rich (foamy) macrophages located in the center. The macrophage-containing center can be further encapsulated by a layer of fibrous tissue with both $\mathrm{B}$ and $\mathrm{T}$ lymphocytes in the peripheral area. Other immune cells like neutrophils and DCs are also present in the granuloma. Granulomas are dynamic and heterogeneous structures with immune cells constantly trafficking in and out. Individual granulomas at any given time within a single patient might differ in several aspects including bacterial numbers, cellular composition, oxygen and nutrient levels and fibrosis. However, if bacterial growth is controlled, individuals can have granulomas present subclinically for decades (i.e. latent infection) but upon disease progression increased central necrosis is observed. Subsequent granuloma cavitation leads to spillage of bacteria into the airways of the infected individual, enabling transmission to a new host. The strong proinflammatory response and subsequent granuloma formation has long been considered to be beneficial for the host; a view that has been questioned during the last few years $(47,51-52)$. 


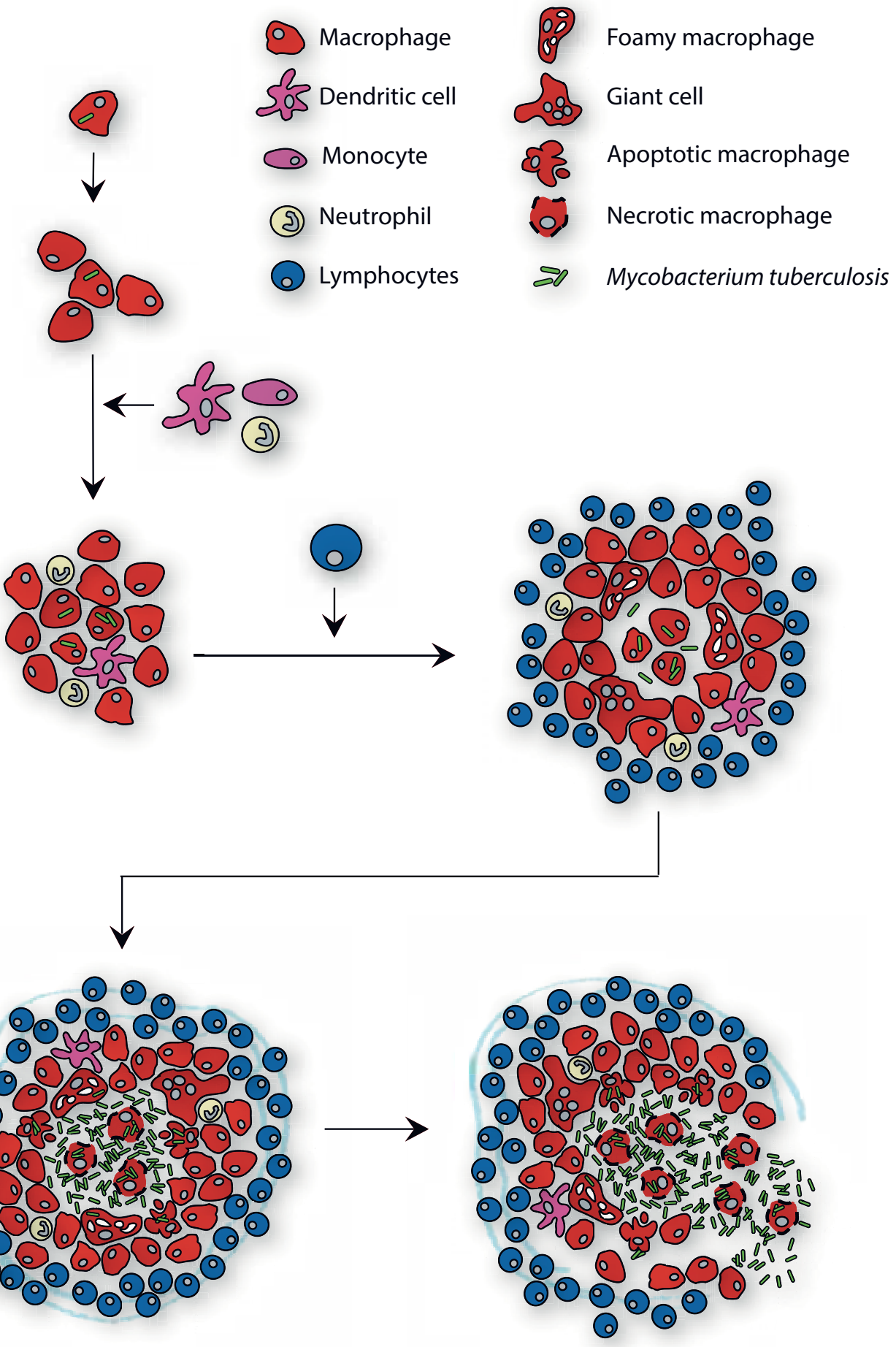


Figure 2. Following uptake of Mycobacterium tuberculosis by an alveolar macrophage (upper left corner), the infected cell initiates an inflammatory response, leading to the recruitment of additional residential macrophages that form a very early granuloma. This early structure is rapidly infiltrated by additional innate cells migrating from adjacent blood vessels. Following the initiation of adaptive immune responses, lymphocytes are recruited from local lymph nodes and at later stages the whole structure can be encapsulated by fibrotic tissue. The bacteria are primarily present in the center of the granuloma where they are continuously being phagocytosed by macrophages. This central region is lined with additional macrophages, including characteristic multinucleated "giant cells" and lipid-rich "foamy" macrophages. As the disease progresses, bacterial replication is no longer controlled and the center of the granuloma starts showing signs of widespread macrophage necrosis. At a critical point, the immune system fails to enclose the infection and the granuloma disintegrates, spilling bacteria into the airways and enabling the infection of new hosts.

\section{Diagnosis and treatment}

Many infectious diseases are readily diagnosed and treated by antibiotics. Some diseases such as smallpox have even been completely eradicated through successful vaccination programs. Fast and reliable diagnostics, efficient treatment and a protective vaccine are ultimate goals in the fight against tuberculosis as well. However, the biology and pathogenesis of $M$. tuberculosis are coupled to several obstacles in achieving these goals. In the following section, diagnosis, treatment and prevention of tuberculosis will be discussed as well innate limitations and drawbacks with the currently used tools.

\section{Diagnosis}

Pulmonary tuberculosis, the most common manifestation of tuberculosis, is characterized by a number of clinical features including chronic cough, weight loss, fever and night sweats (53). In high-endemic regions, the presence of these symptoms together with chest radiography, sputum microscopy and culture are the currently recommended methods for diagnosis of active tuberculosis. Due to their costeffectiveness they are widely used in low-income countries, where the tuberculosis burden is greatest. However, these standard methods lack both in specificity and sensitivity or are very time consuming. Furthermore, they might not be available at the primary health clinics, where patients first seek medical care, nor allow the discrimination between sensitive strains and MDR/XDR tuberculosis (54). Molecular approaches to detecting tuberculosis and antibiotic resistance are routinely used in 
high-income countries, but the cost and the specialized staff and facilities required have been a major obstacle for implementing molecular assays globally.

New and more rapid diagnostic tools are however becoming available also in highendemic countries. WHO endorses several newly developed methods, including Xpert ${ }^{\circledR}$ MTB/RIF technology (8), a real-time PCR-based method where sample preparation, amplification and detection are done in one single cartridge, excluding the need of biosafety facilities or highly trained staff. It also includes probes able to detect rifampicin resistance, which often is coupled with resistance to isoniazid (55). The ability to detect both bacteria and resistance within 100 minutes is expected to shorten the time-to-treatment in both sensitive and MDR cases of tuberculosis and since WHOs endorsement of the technology, 1.1 million tests have been used (8). Other tests endorsed by WHO include the microscopic-observation drug-susceptibility (MODS) assay, which detects $M$. tuberculosis on the basis of cording formation and simultaneously tests for rifampicin and isoniazid resistance. The MODS assay has increased sensitivity and is approximately twice as fast compared to conventional culture methods (56).

Though diagnosing active tuberculosis and identifying transmitting index cases are major objectives in the fight against global tuberculosis, diagnostics of tuberculosis also include the large reservoir of asymptomatic, latently infected individuals. Identifying and treating latent tuberculosis is mainly done in groups where the prevalence of latent infection is high, such as house-hold contacts to patients with active tuberculosis or where the risk of reactivation is high, such as in HIV-positive individuals or in patients receiving immunosuppressant therapy. Since detection of the actual bacteria is rarely possible in latent tuberculosis, testing for latent tuberculosis is done by immunological tests. The most widely used, the tuberculin skin test (TST) has been around for over a century. It is based on the injection of purified protein derivate (PPD) from mycobacteria in the skin of the person to be tested. The subsequent skin reaction is then measured and considered either positive or negative. The major drawback of TST is that PPD contains a crude mixture of antigens non-specific to $M$. tuberculosis, and is therefore unable to distinguish between environmental mycobacteria and $M$. tuberculosis. Furthermore, the test also reacts if the individual has received vaccination against tuberculosis in the form of Bacillus Calmette-Guérin (BCG). To circumvent this, IFN- $\gamma$ release assays (IGRAs) are now used as the gold standard to identify latent tuberculosis. It is based on the recognition of the $M$. tuberculosis-specific antigens ESAT-6 and CFP-10 by peripheral blood mononuclear cells (53). However, due to the increased cost of IGRAs compared to TST, it is not recommended to replace TST with IGRAs in resource-constrained countries. The immunological nature of these tests makes them incapable of separating latent 
infection from active tuberculosis and the reliability is affected in HIV-positive individuals with low CD4+ T cell counts $(8,57)$.

\section{Treatment}

The standard treatment of active tuberculosis consists of an initial phase on a four drug-regimen with the first-line drugs isoniazid, rifampicin, pyrazinamide and ethambutol for two months followed by a continuation phase of four months with only isoniazid and rifampicin. The lengthy treatment is often associated with failure to complete the treatment, increasing the risk of developing antibiotic resistance. In an effort to avoid this, WHO recommends the use of the directly observed treatment, short-course (DOTS) program, where patient compliance is monitored by an independent observer $(8,58)$. In cases of MDR tuberculosis, the recommendation is a specialized regimen of at least four different second-line drugs for 18 months. For latent tuberculosis, a preventive therapy with isoniazid for nine months has long been the standard, but combination therapy with isoniazid and rifapentine for three months has been shown to be as effective as the nine-month regimen (59). The emergence of MDR strains means that future control of tuberculosis requires new and more effective drugs active against $M$. tuberculosis. Fortunately, several new drugs specifically designed against $M$. tuberculosis are currently in the development pipeline, including the promising ATP synthase inhibitor TMC-207, which increases sputum conversion in MDR patients (53).

\section{Vaccination}

In order to reach WHOs 2050 Millennium Development Goal of eradicating tuberculosis, improved diagnostics and improved chemotherapy is not sufficient. To reduce the global incidence, the preventive actions of an effective vaccine is needed (8). The current vaccine against tuberculosis, BCG, is the most widely used vaccine throughout history with four billion doses being administered since it was first described in 1927. It is a live attenuated strain of M. bovis, which is effective in preventing disseminated forms of tuberculosis in children. However, the efficacy of preventing transmittable pulmonary tuberculosis in adults is low. The development of new vaccines against tuberculosis has proven to be challenging for several reasons. The intracellular nature of $M$. tuberculosis requires a robust cellular immune response, where most successful vaccines boost humoral immunity. The fact that $M$. tuberculosis expresses different antigens during the different stages of its lifecycle, and our poor understanding of what factors truly correlates with protective immunity in tuberculosis have thwarted the development of a new vaccine. Additionally, since latent tuberculosis might reactivate in immunocompromised hosts (e.g. HIV-positive individuals), a new vaccine will have to not only confer life-long control but also sterilizing immunity against the bacteria. Much alike the antimycobacterial drugs, 
there has been an enormous development in the field of tuberculosis vaccines in the last decade. Twelve individual vaccines are currently being tested in the field and consist of both new live vaccines and booster vaccines to complement BCG vaccination (60).

\section{Pathogen recognition in tuberculosis}

Innate immunity consists of both resident tissue cells such as macrophages, mast cells and denditric cells, and of circulating cells like monocytes and neutrophils. Their role is to detect and react upon danger signals from invading pathogens or tissue damage and to initiate an appropriate inflammatory response. These signals are referred to as pathogen-associated molecular patterns (PAMPs), which are conserved structures shared by many pathogens, such as microbial nucleic acid, carbohydrates, or damage/danger-associated molecular patterns (DAMPs), e.g. ATP released from dying cells. The signals are detected by a large group of surface-expressed and intracellular receptors, collectively referred to as pathogen recognition receptors (PRRs). Many PPRs act primarily as signaling receptors, but some of them are also involved in the phagocytic uptake of the bacterium. In the following section, some of the most important PRRs in human macrophages capable of recognizing $M$. tuberculosis will be discussed.

\section{C-type lectins and complement receptor 3}

The mannose receptor (MR) is a carbohydrate-binding receptor that is part of a large superfamily of proteins known as C-type lectins. It is expressed at the surface of human macrophages and dendritic cells and, as the name implies, it is able to recognize the mannose-capped ManLAM (61). Since ManLAM is present primarily on virulent bacteria, this route of uptake is mainly coupled to virulent strains including $M$. tuberculosis (62). However, if different ManLAM-expressing strains are compared, a difference in MR-mediated uptake can be seen, suggesting that there is a subtle heterogeneity in ManLAM between strains or that the MR is able to recognize closely related PIMs with mannose cap-like structures (63-64). MR lacks cytoplasmic signaling motifs and upon recognition of ligands, MR does not seem to induce a signal on its own, but rather facilitate the recognition of the ligand by other receptors (65). Binding of mycobacteria to MR can modulate the immune response by increased levels of IL-8 or decreased levels of IL-12 (66-67). More importantly, uptake of $M$. tuberculosis through MR does not lead to killing of the bacterium in human macrophages (68) or reduced bacterial numbers in vivo in mice (69). Instead, MRmediated uptake seems to enhance the ability of the bacteria to inhibit fusion between 
the phagosome and lysosomes, which is an important part of the cell's antimicrobial function (70).

Another receptor important for uptake of bacteria including $M$. tuberculosis is complement receptor 3 (CR3). It belongs to the integrin superfamily and is expressed on innate phagocytic cells where it recognizes a range of ligands including complement fragment iC3b. It is the most important complement receptor for uptake of $M$. tuberculosis and accounts for most of the phagocytosis of complementopsonized mycobacteria (71), but can also bind to and induce phagocytosis of nonopsonized bacteria (72). In contrast to other complement-opsonized preys, which induce an increase in intracellular calcium concentration upon uptake through CR3, complement-opsonized $M$. tuberculosis uptake through CR3 does not increase intracellular calcium concentrations. This calcium inhibition is coupled to decreased phagolysosomal fusion and increased survival of the bacteria (73). Non-opsonic uptake of M. tuberculosis by CR3 is thought to play a role in the very initial uptake by alveolar macrophages, where complement components are absent. However, nonopsonic uptake of $M$. tuberculosis by CR3 does not lead to intracellular killing in macrophages (74).

Dendritic Cell-Specific Intercellular adhesion molecule-3-Grabbing Non-integrin (DCSIGN) is another C-type lectin which has been studied in the context of mycobacterial infections. It was first described as a receptor for HIV, but has been shown to recognize a range of pathogens. It is mainly expressed on the surface of DCs, but can also be found on certain subsets of macrophages including alveolar macrophages (75). DC-SIGN, as MR, recognizes ManLAM from virulent strains of mycobacteria and is the major receptor for uptake of M. tuberculosis in DCs. Interestingly, DCs also express MR and CR3, but these are to a large extent neglected by the bacteria when interacting with DCs. Though the intracellular fate of M. tuberculosis differs between human macrophages and DCs, perhaps due to the difference in uptake routes, binding and uptake through DC-SIGN can have an anti-inflammatory effect with increased production of IL-10 (76).

A third C-type lectin implicated during mycobacterial infection is dectin-1. It is expressed on several different cell types including macrophages, DCs and neutrophils. It recognizes $\beta$-glucans and has been extensively studied in fungal infections, where it contributes to phagocytosis and production of inflammatory cytokines (77). It is still not entirely clear which mycobacterial ligands are recognized by dectin- 1 , but dectin- 1 together with Toll-like receptor (TLR) 2 increases the production of TNF during mycobacterial infection (78). Stimulation of dectin-1 with $\beta$-glucans during mycobacterial infection also leads to increased levels of intracellular reactive oxygen species (ROS) and inhibits intracellular growth of mycobacteria (79). However, this is only seen with non-virulent bacteria, since neither TNF production nor intracellular 
growth in $\beta$-glucan stimulated cells is affected by virulent $M$. tuberculosis (78-79), suggesting that virulent mycobacteria modulate binding to or signaling by dectin-1. This observation is strengthened by in vivo studies in dectin-1 knock-out mice, where dectin-1 have little effect on the inflammatory response or outcome of the infection (80).

\section{Toll-like receptors}

Toll-like receptors are the true archetypes of PRRs and play a crucial role in the initiation of pathogen-induced inflammatory responses. The discovery of TLRs comes from the discovery of the Toll gene in Drosophila and these receptors are highly conserved in both vertebrates and invertebrates. In human innate immune cells, there are 10 TLRs, each with their own ligand specificity and cellular localization. TLR3, TLR7, TLR8, TLR9 are usually present in intracellular compartments, such as endosomes, while TLR1, TLR2, TLR4, TLR5, TLR6 and TLR10 are cell-surface receptors. All TLRs are membrane receptors with an N-terminal ligand binding domain protruding into the extracellular space or endosomal compartment, a transmembrane helix and a C-terminal signaling domain. The N-terminal domain of all TLRs consists of a number of identical motifs named leucine-rich repeats (LRR), which form a horseshoe-shaped structure and is responsible for ligand recognition (81). Generally, the cell-surface TLRs are more involved in the recognition of bacteria, fungi and parasites, while the endosomal members detect viral ligands. TLR4, together with co-receptors, detects lipopolysaccharide (LPS) from Gram-negative bacteria, while TLR2, in concert with either TLR1 or TLR6, detects a range of PAMPs including peptidoglycan, fungal zymosan and LAM from mycobacteria. TLR5 recognizes flagellin from bacterial flagella and the endosomal TLR9 can detect unmethylated CpG motifs of microbial DNA (82).

Upon interaction between a TLR and its ligand, the receptor initiates a signaling event through its cytosolic Toll/IL-1 receptor (TIR) domain. As the name implies, the TIR domain is also found in members of the IL-1 receptor family and usually interacts homotypically with other TIR domains in associated adaptor proteins. The TIR domain of all TLRs, except TLR3, activates the adaptor protein MyD88 directly or through additional adaptors. MyD88 then recruits members of the interleukin-1 receptorassociated kinase (IRAK) family and the signal is relayed downstream to transcription factors, such as interferon regulatory factors (IRFs), mainly involved in the response to viral infections, and nuclear factor kappa B (NF- $\kappa B$ ) (83). NF- $\kappa B$ consists of the subunits p50 and p65, and in resting cells, NF- $\mathrm{BB}$ is bound to its endogenous inhibitor

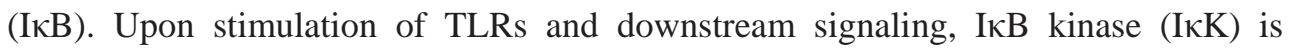
activated and promotes phosphorylation of $\mathrm{I} \kappa \mathrm{B}$, releasing I $\mathrm{\kappa B}$ from NF- $\kappa \mathrm{B}$ and

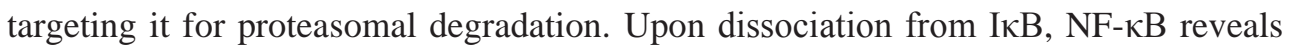


its nuclear translocation domain and enters the nucleus where it exerts its functions. NF- $\kappa B$ induces the activation and transcription of over 150 genes with a wide range of effects, including many proinflammatory cytokines (84).

Mainly TLR2, TLR4 and TLR9 are involved in sensing M. tuberculosis. TLR2 senses several mycobacterial glycolipids including LAM and PIM precursors as well as the mycobacterial $19 \mathrm{kDa}$ lipoprotein, which can be released in the form of membrane vesicles (85). TLR4 recognizes heat-shock proteins released from the bacteria (86), while TLR9 responds to mycobacterial DNA (87). The fact that TLRs sense bacteria and induce production of proinflammatory cytokines in vitro is clear, while the actual importance of the subsequent events is somewhat controversial. Some in vivo experiments using knock-out mice and BCG or mycobacterial antigens have shown that TLR2 is important for control of bacterial growth and TLR4 and TLR9 for the ability to mount an effective Th1 response (88-89). However, low-dose infection with virulent $M$. tuberculosis in mice deficient in either TLR2 or TLR4 does not lead to increased susceptibility or decreased immune responses (90). Since several TLRs are involved in sensing $M$. tuberculosis, it would be possible that the loss of a single TLR could be compensated by other TLRs, but mice that lack all three TLRs associated with mycobacterial recognition (TLR2, TLR4 and TLR9) still control bacterial growth and are able to mount an IFN- $\gamma$ dependent antibacterial response (91). In contrast, mice deficient in the adaptor protein MyD88 quickly succumb to mycobacterial infection, showing that MyD88 is crucial for resistance to M. tuberculosis, but perhaps through its ability to relay signals from other receptors e.g. members of the IL-1 receptor family (91-92). The exact role of TLRs in human tuberculosis still needs to be elucidated, but studies have shown a direct antimicrobial effect upon TLR2 stimulation in human alveolar macrophages (93). A recent meta-analysis of studies concerning two of the most widely studied polymorphisms in the TLR4 gene (D299G and T399I) and susceptibility to tuberculosis showed no association between these polymorphisms and the disease (94). In contrast, another recent meta-analysis showed a difference in susceptibility to tuberculosis associated with common polymorphisms in both TLR2 (G2258A) and its associated TLRs, TLR1 (G1805T) and TLR6 (C745T) (95). Though not all possible single-nucleotide polymorphisms (SNPs) were investigated and the number of studies included in the meta-analyses was rather limited, this suggests that TLR2 signaling has a greater impact in human tuberculosis susceptibility than TLR4 signaling.

\section{Nucleotide-binding oligomerization domain-like receptors}

Nucleotide-binding oligomerization domain-like receptors (NLRs) are a family of intracellular receptors containing over 20 different members. NLRs are highly conserved and mammalian NLRs bear resemblance to a subgroup of disease-resistance genes in plants. The common denominators in all NLRs are the LRR domain, which 
they share with TLRs, and a central nucleotide-binding NACHT domain, hence the name of the family. NLRs can be further divided into subfamilies depending on their $\mathrm{N}$-terminal effector domain. Though at least four different subfamilies have been identified, two of them are the major sensors involved in pathogen recognition; the NLRCs, with an N-terminal caspase recruitment domain (CARD) and the NLRPs, with an N-terminal pyrin domain (PYD) (96). The different protein domains of the NLR interact with each other, keeping the non-active NLR in an autoinhibited and closed conformation until binding of the ligand (97-98). This suppressive, but signalcompetent, conformation has been proposed to be stabilized by the regulatory proteins SGT1 and heat-shock protein 90 (HSP90) (99). Upon ligand recognition by the LRR domain, the regulatory proteins dissociate and the autoinhibitory conformation is changed, exposing the central NACHT domain, and the NACHT domains of several identical NLRs undergo homotypic oligomerization in an ATP-dependent manner, facilitating the recruitment of downstream adaptor proteins (100-101). The general structure and activation of NLRs is depicted in Figure 3.

Among the NLRCs, the most studied members are Nod1 and Nod2. Nod1 and Nod2 primarily recognize fragments of peptidoglycan, a major constituent of the bacterial cell wall. More specifically, Nod2 senses muramyl dipeptide (MDP), present in virtually all bacteria, while Nod1 recognizes diaminopimelic acid (DAP), an unusual amino acid mainly found in Gram-negative bacteria (102). When activated, both Nod1 and Nod2 oligomerize and recruit the kinase RICK/RIP2 through CARD-CARD interaction, forming what is sometimes referred to as a Nodosome. RICK/RIP2 in turn

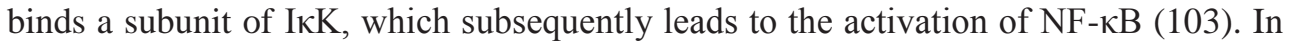
addition to NF-kB activation, both Nod1 and Nod2 can induce autophagy, a conserved system for degradation of cytoplasmic content, in a RICK/RIP2-independent manner (104-105). In tuberculosis, the recognition of mycobacterial MDP by Nod2 has been implicated to play a role. Virulent $M$. tuberculosis activates Nod2, leading to the generation of type I interferons (IFN- $\alpha /$ IFN- $\beta$ ) (106) and silencing of Nod2 in human macrophages decrease cytokine production and increases bacterial growth (107). Studies in vivo have demonstrated that Nod2, despite adding to the production of proinflammatory cytokines, is redundant early in the course of the infection, while contributing to lower bacterial numbers during later stages (108-109).

The NLRPs are the largest of the NLR subfamilies with 14 members identified. They are expressed in various tissues and most of them can be found in cells of the reproductive organs (110). NLRP1b was the first of the NLRPs to be discovered and was identified due to its pyrin domain. The pyrin domain is a member of a larger death domain-fold superfamily, whose members typically interact with other identical domains e.g. PYD-PYD or CARD-CARD interactions (111). It was later discovered that NLRP1b, together with other proteins, was able to form an intracellular protein 


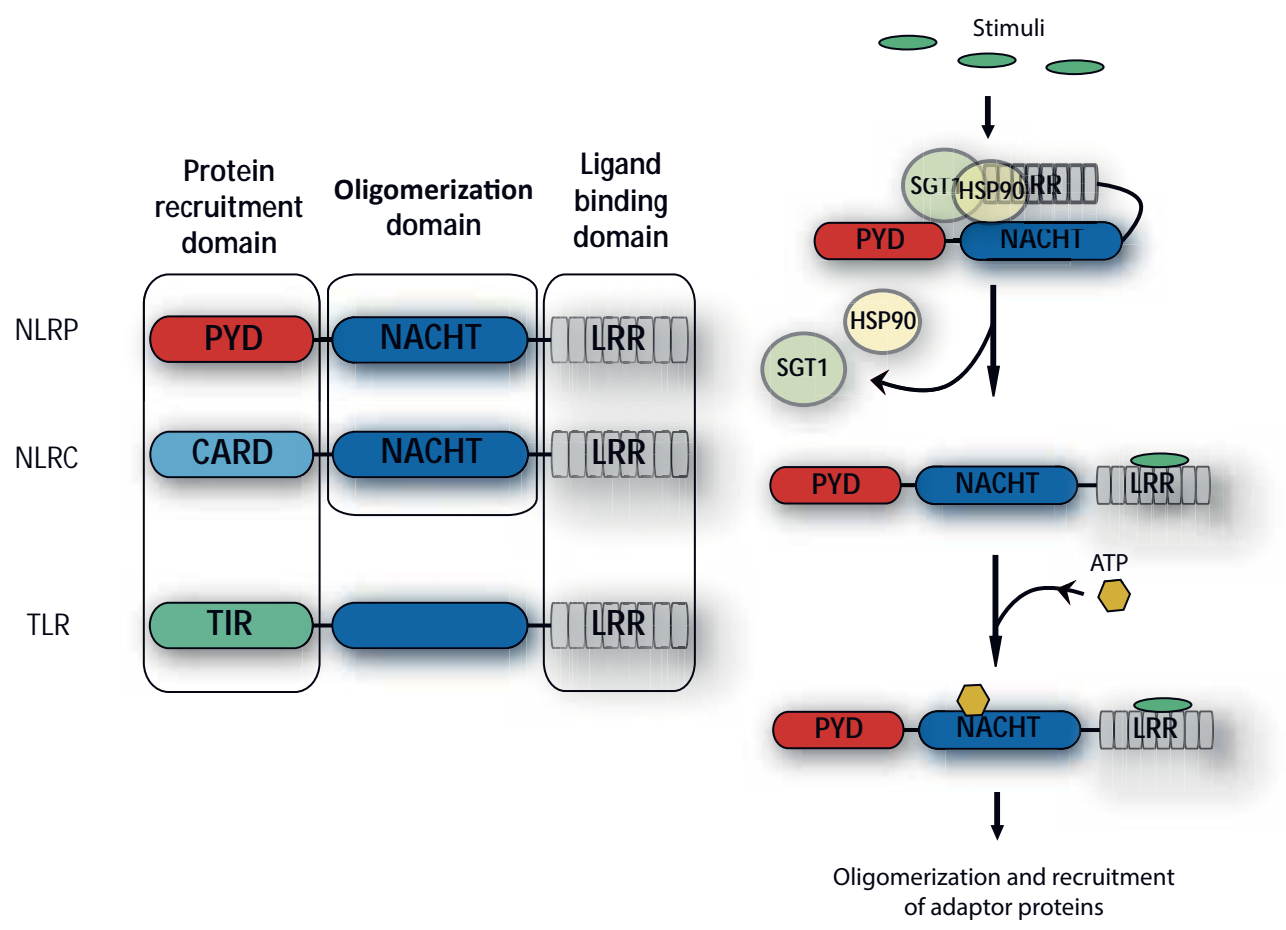

Figure 3. The left part of the figure shows the functional protein domains of two different subfamilies of NLRs with the basic structure of a TLR for comparison. The C-terminal LRR domain is thought to be involved in ligand recognition, the central NACHT domain in oligomerization of several identical NLRs, while the N-terminal domain recruits additional adaptor proteins. The right part of the figure shows a hypothetical model for activation of NLRs. The NLR is kept in a suppressive, but signaling-competent state by the proteins SGT1 and HSP90. Upon recognition of a stimulus, these regulatory proteins dissociate and the NLR assumes an open conformation enabling ATP-dependent oligomerization and recruitment of adaptor proteins.

complex, termed inflammasome, responsible for the processing of proIL-1 $\beta$ into its active form (112). NLRP1b mainly reacts to anthrax lethal toxin (LT) (113) and a potential role in tuberculosis is still to be shown. NLRP3 represents the most studied NLRP and like NLRP1b, NLRP3 also forms an inflammasome (114). However, NLRP3 shows a more restricted expression pattern than NLRP1b and is mainly found in immune cells (115), suggesting a more central role in immunity and inflammation. This is further highlighted by the large number of NLRP3 activators so far identified. NLRP3 responds upon endogenous DAMPs like extracellular ATP and monosodium urate, environmental irritants such as silica and asbestos and PAMPs such as poreforming toxins, intracellular bacteria and virus (116). Inflammasome assembly and the role of NLRP3 during tuberculosis will be discussed in later chapters. 
The understanding of the role of other NLRs in immune responses during infections is still incomplete but non-inflammasome NLRPs such as NLRP6, NLRP10 and NLRP12, which are also expressed in myeloid cells, are involved in the regulation of NF- $\kappa B$ activation, IL-1 $\beta$ release, antigen presentation and intestinal homeostasis (117). Genetic studies in humans have clearly shown that polymorphisms in NLRs can be tightly linked to a number of inflammatory disorders $(114,118)$, but except for a few studies conducted on common polymorphisms in the Nod2 gene (119), the impact of NLR polymorphisms in infectious diseases and tuberculosis is still unknown.

\section{Macrophage effector functions and Mycobacterium tuberculosis}

Following recognition and uptake of $M$. tuberculosis, the host cell initiates a number of responses to limit bacterial replication and spread with the ultimate goal of killing the bacteria, thus eradicating the infection. Most pathogenic bacteria have numerous ways to counteract these responses and $M$. tuberculosis is particularly adept in interfering with macrophage function. The following section will highlight different macrophage functions, their role in tuberculosis and how they are affected by the bacterium.

\section{Cytokine response}

Host defense against all invading pathogens, as well as the subsequent resolution of inflammation, rely on the induction and the effects of a plethora of cytokines. Many of them are produced by the human macrophage and act both as a link to the adaptive immunity and to enhance macrophage effector functions. Many cytokines play a role when mounting an appropriate immune response against $M$. tuberculosis. However, this section will focus on those cytokines that are produced by the macrophage or are critical for macrophage function. TNF, IFN- $\gamma$ and the interleukins IL-6, IL-10 and IL12 will be discussed. The members of the IL-1 family and especially IL-1 $\beta$ will be discussed in later chapters.

TNF is produced mainly by macrophages upon NF-kB activation e.g. through TLR stimulation and plays a part in a number of different processes including programmed cell death (apoptosis) and the recruitment and activation of immune cells (120). The effects of TNF on macrophages are beneficial for the host by increasing mycobacterial killing in the cell (121). M. tuberculosis is able to decrease the stability of TNF mRNA in human macrophages and to actively inhibit secretion of the protein (122-123). The recruitment of cells through TNF is important for granuloma formation and 
maintenance, and mice lacking TNF display disorganized granulomas and quickly succumb to infection (124). The importance of TNF signaling during tuberculosis has also been demonstrated in recent years by the fact that biological anti-TNF agents, used to treat autoimmune diseases, increase the risk of developing tuberculosis and dissemination of the disease (125). Despite a clear protective role, dysregulated and excessive production of TNF can lead to chronic inflammation and wasting (120) and result in host-detrimental immunopathology during mycobacterial infections (126).

In experimental tuberculosis, a strong Th1-dominated immune response with IFN- $\gamma$ producing CD4+ T cells is necessary for protection. In murine macrophages, IFN- $\gamma$ has many effects, including upregulation of inducible nitric oxide synthase (iNOS), which in turn generates nitric oxide (NO) and reactive nitrogen species (RNS) capable of killing M. tuberculosis (127). The necessity of IFN- $\gamma$ has also been clearly demonstrated in vivo, where mice lacking IFN- $\gamma$ are extremely sensitive to tuberculosis, displaying both increased tissue destruction and bacterial load (128). To counteract this important cytokine, $M$. tuberculosis, through its $19 \mathrm{kDa}$ lipoprotein and certain cell wall components, has the ability to inhibit the effects of IFN- $\gamma$ on macrophages e.g. the induction of specific IFN- $\gamma$ responsive genes (129). The importance of IFN- $\gamma$ in humans was early shown by the finding that individuals, who have deficiencies in IFN- $\gamma$ signaling, have increased susceptibility to mycobacterial infections (130). However, the ability to induce mycobacterial killing in human macrophages through IFN- $\gamma$ in vitro has been more of a theoretical idea than a wellestablished phenomenon. Recently this has been clarified by studies showing that human macrophages stimulated with IFN- $\gamma$ also require the presence of active vitamin $\mathrm{D}$, which leads to increased expression of antimicrobial peptides, increased phagolysosomal fusion and enhanced killing of M. tuberculosis (131). Due to its crucial role in tuberculosis, IFN- $\gamma$ has commonly been used as a marker for effective immunity against tuberculosis, but the correlation between the levels of IFN- $\gamma$ and protection is poor (132).

IL-12, IL-6 and IL-10 are all produced by professional phagocytes, such as macrophages, and these cytokines play a role during tuberculosis. The main role of IL12 in tuberculosis is to stimulate the release of IFN- $\gamma$ from CD4+ T cells, and both mice and humans deficient in either IL-12 signaling or expression of the p40 subunit are more susceptible to mycobacterial infections. Mycobacterial LAM can also inhibit TLR-induced IL-12 in macrophages, which decreases the IFN- $\gamma$ production of the host (133). The anti-inflammatory cytokine IL-10 can exert its function directly on macrophages and counteracts the effects of both TNF and IL-12, leading to lower IFN$\gamma$ levels and less responsive macrophages. IL-10 can block phagosomal maturation in infected human macrophages and mice lacking IL-10 are more resistant to tuberculosis. Furthermore, hypervirulent strains of $M$. tuberculosis induce increased 
levels of IL-10, suggesting that the bacterium uses this endogenous signal to its own advantage (134). On the other hand, during later stages of $M$. tuberculosis infection in mice, IL-10 seems to play a protective role by decreasing pulmonary inflammation and tissue damage (135), showing that a complete loss of IL-10 is detrimental to the host. IL-6 is a pleiotropic cytokine affecting a number of tissues and is produced by macrophages infected with $M$. tuberculosis. Mice lacking IL-6 have a higher bacterial burden in their lungs and impaired IFN- $\gamma$ production early in the infection, suggesting a beneficial role for IL-6 in tuberculosis (136). As opposed to this, IL-6 can also inhibit IFN- $\gamma$ stimulated gene transcription in macrophages, indicating that $M$. tuberculosis might benefit from the production of IL-6 (137). Though the importance of IL-6 in tuberculosis is still not completely understood, it is not crucial for protection against tuberculosis and supposedly plays a more regulatory role.

\section{Macrophage activation and polarization}

Being resident in tissues, the human macrophage does not only function as the first line of defense against infections, but also play a part in tissue homeostasis and remodeling. To execute these diverse functions, a great deal of plasticity is required and macrophages can be differentiated into the "classically activated" phenotype (M1) and the "alternatively activated" phenotype (M2). M1 macrophages are induced by a typical Th1 milieu, such as IFN- $\gamma$ and TLR ligands, while M2 is induced by a number of stimuli, including Th2 cytokines. The M1 macrophage further drives a Th1 response, produces proinflammatory cytokines and high levels of NO (at least in mice) and is well adapted to handle microbes. The M2 macrophage, on the other hand, is more involved in the protection against parasites, has a high phagocytic capacity with high expression of scavenger receptors and acts as a regulator of the immune response (138). The M2 phenotype can be further divided into subtypes depending on the stimuli and specific effector functions. The M2a (alternative) subtype is mainly induced by IL-4 and IL-13, produces IL-10, IL-1 receptor antagonist (IL-1Ra) and is implicated in parasite control and allergy. M2b (type II) macrophages are induced by immune complexes together with IL-1 receptor (IL-1R) or TLR agonists. The proinflammatory stimuli for M2b macrophages are reflected in their cytokine production, which is characterized by both proinflammatory cytokines like TNF and IL-6 as well as high levels of anti-inflammatory IL-10 (coupled with low IL-12 production). The mixed cytokine profile of M2b macrophages gives them an immunoregulatory function. The third M2 subtype, M2c (deactivated), is induced primarily by IL-10 and also produces IL-10 together with another anti-inflammatory cytokine, transforming growth factor $\beta$ (TGF- $\beta$ ), which has immunosuppressive effects and is involved in tissue remodeling (139). The different subtypes of M2 as well as M1 macrophages can be distinguished by the expression pattern of a number of surface markers. The strict classification of macrophages into these categories has been 
questioned and might not represent the true plasticity of these cells (140). It is very likely that an infinite number of macrophage phenotypes exist between the M1 and M2 extremes and that in vivo, macrophages are constantly adapting to meet the specific challenges at any given time point.

An efficient immune response against $M$. tuberculosis is foremost dependent on M1 macrophages induced by IFN- $\gamma$ and recognition of the bacterium, but as a part of a successful immune response, anti-inflammatory factors and M2 macrophages are needed to carefully balance inflammation and avoid unnecessary tissue damage and pathology. However, these protective actions can be exploited and promoting M2 macrophages, while inhibiting M1 effector mechanisms would be beneficial for the bacteria. As mentioned earlier, $M$. tuberculosis can actively inhibit the actions of IFN$\gamma$ and induction of an M1 program by interfering with the cytokine network. The beneficial effect for the bacteria of inducing a more Th2-dominated response is shown by the fact that M2 macrophages are less responsive to IFN- $\gamma$ and produce less NO during infection (141). The same diminished NO production can be seen in the lungs of transgenic mice with macrophage-specific overexpression of IL-10 (i.e. M2 macrophages). These mice succumb faster to the infection and have higher bacterial burden compared to control mice (142). In humans, Th2 cytokines are produced during active disease, and patients undergoing successful treatment have a transcriptional switch from a Th2- to a Th1-dominated expression profile in pulmonary cells (143), strengthening the idea that $M$. tuberculosis to some extent skews the local immune response towards a more beneficial Th2/M2 milieu.

\section{Phagosomal maturation}

After phagocytic uptake, which in itself is a complex network of signaling cascades and actin rearrangement events (144), the bacterium ends up in an internalized phagosome. This phagosome will through a number of interactions with other parts of the intracellular trafficking and transport machinery (endocytic pathway) acquire different proteins and markers until it finally fuses with a lysosome. (145). The regulation of phagolysomal maturation is largely dependent on two families of proteins; Rab GTPases and SNAREs. Rab proteins function primarily as tethering and docking proteins, thereby enabling the SNAREs to actually fuse the membranes of the phagosome and endosome/lysosome (146).

The initial phagosomal membrane is identical to the plasma membrane of the cell but shortly after internalization it interacts with early endosomes. These early endosomes, or sorting endosomes, have the ability to direct the ingested prey back to the plasma membrane through recycling endosomes or for further degradation by redirecting it to late endosomes. Early endosomes are mildly acidic (pH around 6) and are identified by the presence of Rab5 and the early endosomal antigen 1 (EEA1) protein (145). Rab5 
and EEA1 enable the early endosome to interact with the SNARE protein syntaxin 6 on target membranes, leading to fusion of the membranes (147). The early Rab5/EEA1-containing phagosome develops into a late phagosome, which has acquired a different set of proteins, such as lysosomal-associated membrane proteins (LAMPs), hydrolytic enzymes and has a lower $\mathrm{pH}$ compared to the early phagosome (145). A hallmark during the progression from an early to a late phagosome is the so called Rab-switch, where Rab5 is replaced by Rab7 as the phagosome makes its way from the periphery of the cell towards more centrally located lysosomes (148). This switch has been proposed to be initiated by the activation of Rab7 by Rab5. Rab7 subsequently activates more Rab7, while simultaneously suppressing and removing Rab5 (149). The late phagosome undergoes several transient fusions, so called "kissand-run", and thereafter full fusions with additional late endosomes and lysosomes until it finally becomes a fully matured phagolysosome (150).

After the final step of fusing with lysosomes, the phagolysosome has acquired several characteristics such as low $\mathrm{pH}$ (4.5-5.5), hydrolytic enzymes and high levels of ROS, all designed to aid in the degradation of the ingested prey. The lowering of $\mathrm{pH}$ during the process of phagosomal maturation is accomplished through the continuous recruitment of vacuolar ATPases. The vacuolar ATPase consists of two major complexes; $\mathrm{V}_{1}$ complex and $\mathrm{V}_{0}$ complex. The $\mathrm{V}_{1}$ complex hydrolyses ATP, enabling the $\mathrm{V}_{0}$ complex to pump $\mathrm{H}^{+}$into the lumen of the phagosome, lowering its $\mathrm{pH}$. This acidification in itself leads to an unfavorable environment for many ingested microbes and is a requirement for the full activation of the cathepsin family of acidic proteases, which are important for the antimicrobial function of the phagolysosome (151). These proteases are joined by a number of additional proteins that either deprive the microbe of essential nutrients required for bacterial growth or have direct antimicrobial effects. Examples of the previous include the scavanger lactoferrin, which sequesters iron in the phagolysosomal lumen, and natural resistance-associated macrophage protein 1 (NRAMP1), which can actively transport metal ions, such as $\mathrm{Fe}^{2+}$ and $\mathrm{Zn}^{2+}$ from the lumen with the help of the proton gradient created by the vacuolar ATPase. Direct antimicrobial effects are accomplished by small antimicrobial peptides, such as defensins and cathelicidins, which both induce permeabilization of bacterial membranes. The maturing phagosome also sequentially acquire a number of additional peptidases, lipases and carbohydrate-degrading hydrolases (152). The antimicrobial function of the phagolysosomal lumen is further enhanced by the generation of oxygen-derived and nitrogen-derived radicals. Superoxide $\left(\mathrm{O}_{2} \bullet \bullet\right)$ is generated by the multimeric enzyme phagocyte NADPH oxidase (NOX2), which assembles in the membrane of the phagolysosome. The generated superoxide can further react with $\mathrm{H}^{+}$ to form additional ROS, including hydrogen peroxide $\left(\mathrm{H}_{2} \mathrm{O}_{2}\right)$ and hydroxyl radicals $(\bullet \mathrm{OH})$. The dimeric iNOS is also located to the membrane of the phagolysosome, where it produces nitrogen oxide (NO•), which in turn can generate additional RNS, 
such as nitrogen dioxide $\left(\mathrm{NO}_{2}{ }^{\bullet}\right)$ and peroxynitrite $\left(\mathrm{ONOO}^{-}\right)$. All these compounds are harmful and can cause direct damage to DNA, or damage to proteins and membranes, mainly through interaction with thiol groups, metal centers and oxidation of lipids (153).

The importance of proper phagosomal maturation during intracellular infection by $M$. tuberculosis is indisputable. Loss of regulators of phagolysomal fusion leads to a complete halt of the maturation process and failure to deliver phagosomal content to lysosomes (154). Individuals with deficiencies in different effector mechanisms connected to a functional phagolysosome, such as low expression of NRAMP1 or nonfunctional NOX2, have increased susceptibility to mycobacterial infection (155-156). To circumvent the antimicrobial function of the phagolysosome and avoid degradation, M. tuberculosis has evolved numerous ways to inhibit phagolysosomal maturation and neutralize many of the toxic effects. The bacteria can maintain a proper intrabacterial $\mathrm{pH}$ and secrete ammonia to survive in acid environments, while simultaneously inhibiting the accumulation of vacuolar ATPase in the phagosome by secreting a tyrosine phosphatase (PtpA) (157-159). The decreased recruitment of vacuolar ATPase does not only lead to an increased $\mathrm{pH}$ per se but also inhibits the function of those enzymes requiring acidic conditions, for example cathepsin $\mathrm{D}$, which is important for controlling intracellular growth of M. tuberculosis (160). Additionally, LAM from $M$. tuberculosis insert itself into membrane rafts of the macrophage cell membrane (161), where it interferes with signaling events preceding the recruitment of EEA1, in turn required for the interaction and fusion with late endosomes. Mycobacterial phagosomes are therefore severely retarded in the acquisition of Rab7 and retain Rab5 (162). Another mycobacterial molecule secreted, acid phosphatase M (SapM), also interferes with the same phosphatidylinositol 3-phosphate-dependent signaling (163), thereby inhibiting EEA1 recruitment. To protect itself from radicals and redox stress, $M$. tuberculosis has sensors capable of registering the surrounding levels of radicals, oxygen and nitric oxide, namely the kinases DosS and DosT. The information from DosS and DosT feeds into a response regulator, DosR, which in turn induces the transcription of a number of genes collectively known as the DosR regulon. Though the DosR regulon is mainly implicated in the induction of dormancy in the bacteria, the regulon also includes genes for redox homeostasis. In addition, $M$. tuberculosis also expresses antioxidants, such as superoxide dismutase (SOD), catalase (KatG) and alkyl hydroperoxidase (AhpC), which can neutralize ROS (164). Finally, to overcome deprivation of essential iron, M. tuberculosis expresses and releases the siderophore mycobactin, which is a high-affinity iron chelator, whose action is dependent on the secretion system ESX-3 (165).

Enhancing the normal functions of the mature phagolysosome and overcoming the protective countermeasures of the bacteria can be accomplished by stimulation of the 
macrophage, thus creating an "arms race" or a tug-of-war between the host cell and the bacterium. Stimulation of infected macrophages by the proinflammatory cytokines IL$1 \beta$, TNF and IFN- $\gamma$ have been shown to lead to an increase in phagosomal acidification as well as increased acquisition of late endosomal markers LAMP-1 or LAMP-3/CD63 to the phagosome (166-168). As mentioned above, proinflammatory cytokines do not only drive the maturation of the phagosome, but also increases production of NO and expression of antimicrobial peptides (121, 131). In addition to proinflammatory cytokines, other stimuli can increase the functions of the phagolysosome. For example, in human macrophages, an increase in antimicrobial peptides and increased bacterial killing can be accomplished by a combination of TLR and vitamin D stimulation or by uptake of antimicrobial peptides from apoptotic neutrophils (49-50, 93). Furthermore, enhanced recruitment of vacuolar ATPases to phagosomes containing $M$. tuberculosis in human macrophages can be induced by pharmacological inhibition of the human Abl tyrosine kinase (Abl1), leading to increased acidification and bacterial killing (169).

The vast number of effector functions designed to degrade phagocytosed prey during phagosomal maturation, and the fact that $M$. tuberculosis has ways to circumvent or inhibit most of them, underlines the very central role this process plays in the pathogenesis of tuberculosis. Enhancing the effects coupled to proper phagosomal maturation by different stimuli in murine cells seems to ultimately come down to an increase in iNOS expression and NO production. Though iNOS is expressed in granuloma macrophages and tuberculosis patients display lower levels of NO in exhaled air compared to control subjects (170-171), the role of NO in human macrophages is still not firmly established. It is perhaps the combination of enhancing several of the effector functions of the phagolysosome, including NO production, that lead to increased killing in the human system.

\section{The fate of the mycobacterial phagosome}

Overcoming the mycobacterial block of phagosomal maturation and increasing the acidification and bactericidal functions in the phagosome are crucial for the host cell's ability to handle the infection. However, the extent of macrophage activation and subsequent responses to that stimulation are likely to influence the ultimate fate of the phagosome. In addition to the mechanisms of inhibiting phagosomal maturation as a strategy for survival, $M$. tuberculosis also has the ability to induce a protective stress response triggered by hypoxia and redox stress. This response mainly involves the above mentioned DosR regulon and is thought to lead to a switch from an active to a dormant bacterial phenotype (172). Genes of the DosR regulon are quickly activated after the bacterium enters the macrophage and the expression of these genes increases during the course of the infection. The expression profile of the stress responses is 
thought to be tightly coupled to the environmental changes in the phagosome imposed by the macrophage (173-174). The dormancy program initiated by DosR leads to phenotypic changes characteristic of dormant bacteria including a non-replicating state (175), phenotypic tolerance to antibiotics (176), changes in metabolism (177), accumulation of lipids (178) and alterations to the bacterial cell wall (179). All of these changes prepare the bacterium for a persistent state that helps it survive during hypoxic and less favorable conditions. Dormant bacteria are generally thought to be responsible for causing the asymptomatic, latent infections that can persist for decades. DosR antigens are highly recognized by $\mathrm{T}$ cells from latently infected individuals, suggesting a constant expression of dormancy-associated genes (180). However, DosR is also required for exiting dormancy (181), indicating a constant turnover of the bacterial population in vivo (182). Entering dormancy as a defensive strategy is underlined by the fact that already dormant bacteria infecting human macrophages fail to inhibit phagosomal maturation, but are still not killed by the macrophage (183). In a way, being phagocytosed by a macrophage able to impose stress on the bacterium can be seen as a maturation process of the bacterium itself leading to the induction of dormancy.

An alternative strategy employed by intracellular pathogens to avoid the harmful environment of the mature phagolysosome is to simply escape the phagosomal vacuole and gain access to the nutrient-rich cytosol and replicate more efficiently (152). The close relative to $M$. tuberculosis, $M$. marinum is able to escape from the phagosome and move in the cytoplasm by actin-based motility, a process dependent on a functional ESX-1 system and ESAT-6 secretion (184-185). ESAT-6 is able to form pores and have membrane-lysing properties that are crucial in escaping both from the phagosome and the host cell $(37,184,186)$. In the case of M. tuberculosis, which has been primarily viewed as an intraphagosomal pathogen, analyses of infected human macrophages using electron microscopy have shown that bacteria can be detected in the cytosol. The escape of $M$. tuberculosis has also been shown to be dependent on ESAT-6, and escaping the phagosome increases the replication rate of the bacteria (187). After the publication of this study, the ability of M. tuberculosis to escape the phagosome became a topic of controversy and it has been debated if phagosomal escape only happens in certain model systems, is an epiphenomenon generated as the bacterium exits the cells to spread to adjacent cells or simply an artifact due to experimental procedures (188-190). A recent study using a fluorescence resonance energy transfer approach taking advantage of the intrinsic beta-lactamase activity of mycobacteria have strengthened the initial observations of phagosomal escape of $M$. tuberculosis in human macrophages (191). 


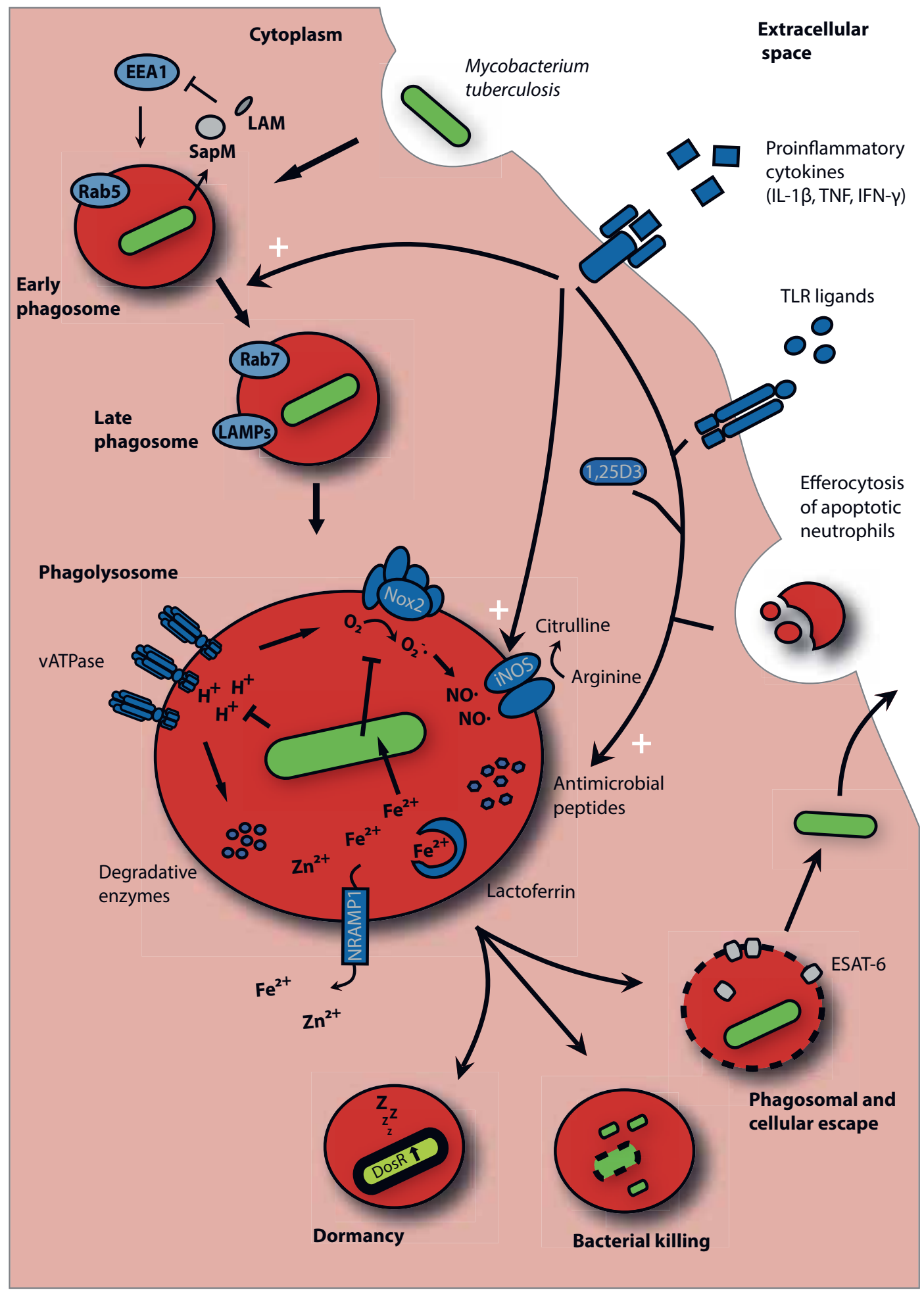


Figure 4. Following ingestion of prey by the macrophage, the formed phagosome undergoes a series of fusion events with early and late endosomes until it eventually fuses with lysosomes to become a mature phagolysosome. During this process, the phagosome gradually acquires a number of antimicrobial effector functions, including low $\mathrm{pH}$, harmful radicals, degradative enzymes, antimicrobial peptides and proteins capable of depriving the microbe of essential nutrients such as metal ions. Mycobacterium tuberculosis has evolved ways of inhibiting maturation of the phagosome as well as mechanisms to counter the bactericidal functions of the mature phagolysosome. These mechanisms include inhibition of radicals, resistance to acidic environments and sequestering iron from the lumen of the phagosome. The maturation of the phagosome and the bactericidal functions of the mature phagolysosome can be further enhanced by host factors like proinflammatory cytokines, activation of the vitamin $D$ receptor (VDR) and uptake of apoptotic neutrophils or stimulation by exogenous agents such as TLR ligands. The balance between protective host factors and bacterial virulence mechanisms ultimately determine the outcome of infection, which includes bacterial clearance, induction of dormancy or phagosomal escape and cell death.

Escape from the phagosome together with the induction of dormancy represents two different non-lethal outcomes of the individual bacterium encapsulated in the phagosome and it is easy to imagine that the outcome is tightly coupled to the interaction with the host cell. Thus, the magnitude of antimicrobial effector functions initiated by the macrophage can be viewed as a spectrum that directly influences the behavior of the bacterium, where bacterial killing in an robust and activated macrophage constitutes one end of the spectrum and phagosomal and cellular escape in weak macrophages representing the other end, with the induction of dormant bacteria in moderately efficient macrophages. However, induction of dormancy or phagosomal escape might not be mutually exclusive. The regulation and subsequent fate of the mycobacterial phagosome is summarized in Figure 4.

\section{Autophagy}

A process that share many similarities with the uptake of extracellular macromolecules through phagocytosis is macroautophagy, or simply autophagy. It is a well conserved process for the capture and degradation of toxic protein aggregates, dysfunctional organelles and intracellular microbes as well as serving as a digestion system for cytoplasmic nutrients in the case of starvation (192). The autophagic machinery consists of a set of autophagy-related (Atg) proteins and can be induced by a number of stimuli including starvation, TLR signaling and proinflammatory cytokines (193). Following stimulation of autophagy, two different protein complexes are sequentially activated; the Ulk1-containing complex which in turn phosphorylates and activates the Beclin-1/hVPS34 complex (194). These complexes integrate the upstream signals and direct downstream Atg proteins. The Ulk1-containing complex is under normal conditions inhibited by another complex that contains the mammalian target of 
rapamycin (mTOR), a common target for inhibition when experimentally inducing autophagy. Activation of the Ulk1-containing and Beclin-1/hVPS34 complexes leads to the recruitment of membrane from intracellular compartments such as the endoplasmic reticulum (ER), mitochondria and Golgi apparatus. The recruited membrane forms a small, crescent-shaped isolation membrane or phagophore with double membranes. To close this crescent and completely encapsulate the cargo or prey, the Atg5-Atg12/Atg16L1 complex is assembled on the phagophore membrane. This complex acts as an enzyme and conjugates phosphatidylethanolamine to microtubule-associated protein 1A/1B-light chain 3 (LC3). Following this conjugation, LC3 goes from being cytosolic (LC3-I) to being membrane bound (LC3-II), where it acts as a crucial mediator of membrane elongation and closure. The hallmark of LC3-I conversion is a commonly studied marker for the induction of autophagsomes (192193, 195-196). Microbes can be more directly targeted for autophagosomal degradation by a certain class of newly discovered PRRs, termed sequestosome 1/p62like receptors (SLRs). These contain ubiquitin-recognition domains, which identify the ubiquitinated cargo, an LC3 interacting domain and additional protein interaction domains. SLRs are able to act as molecular bridges between the microbe and the LC3containing phagophore $(192,197)$. Increased phagosomal maturation by the recruitment of LC3 to conventional phagosomes has also been reported, indicating a cross-talk between the compartments (198). The autophagosome is then transported to lysosomes for degradation in a Rab-dependent manner, much alike the maturation of a regular phagosome (199).

Due to the fact that $M$. tuberculosis is an intracellular pathogen, the protective role of autophagy has received a lot of attention in the last decade. Inducing autophagy in murine macrophages infected with $M$. tuberculosis by stimulating with rapamycin, starvation or IFN- $\gamma$ leads to increased phagosomal maturation and increased bacterial killing (48). The beneficial effect on bacterial killing by vitamin D stimulation can be partly explained by the ability of vitamin $\mathrm{D}$ to induce expression of the antimicrobial peptide cathelicidin, which in addition to its direct antimicrobial functions also stimulates autophagy (200). The presence of vitamin D also enables further induction of the autophagic pathway in human macrophages upon stimulation with IFN- $\gamma(131)$. This can be counteracted by Th2 cytokines, which inhibit IFN- $\gamma$-induced autophagy, leading to inhibition of phagosomal maturation and increased bacterial growth (201). This raises the possibility that the positive effect of IFN- $\gamma$ on phagosomal maturation in human macrophages is through induction of autophagy instead of direct stimulation of phagolysosomal fusion. In addition to the direct encapsulation of cytosolic bacteria or bacterium-containing phagosomes into autophagosomes, autophagy can also indirectly assist in killing of the bacterium. For example, two of the first-line drugs against tuberculosis, isoniazid and pyrazinamide, induce ROS-dependent autophagy and inhibition of autophagy compromise the efficacy of the drugs in murine 
macrophages (202) and autophagy-dependent delivery of ubiquitin-derived antimicrobial peptides (cryptides) to the $M$. tuberculosis-containing phagosome leads to increased killing of the bacteria (203). The in vitro observations showing a protective role of autophagy in tuberculosis have also been confirmed in vivo where myeloid-specific knock-out of Atg5 required for autophagy lead to higher bacterial burden and to increased immunopathology characterized by an IL-17-driven influx of neutrophils (204). In line with that notion, human polymorphisms in the autophagyregulating protein immunity-related GTPase family $\mathrm{M}$ protein 1 (IRGM1) are associated with susceptibility to tuberculosis (205). Though autophagy is protective against $M$. tuberculosis, some studies have reported an inhibition of autophagy by the bacteria. In human DCs, the bacteria can inhibit the fusion between autophagosomes and lysosomes in an ESX-1 dependent manner (206) and autophagosome formation has been shown to be inhibited by an ESAT-6/CFP-10 fusion protein, leading to increased bacterial survival in murine macrophages (207).

A very clear antimicrobial effect of autophagy can be seen in most model systems despite the fact that maturation of the formed autophagosome involves much of the same machinery as the maturation of conventional phagosomes, i.e. Rab proteins (199), and should be susceptible for M. tuberculosis-induced maturation block. The answer to this might lie in the very local effect of the mycobacterial effector molecules responsible for the maturation block and that an effective block requires a closely apposing phagosomal membrane (208), a feature that would be lost upon addition of a double autophagosomal membrane.

\section{Cell death}

An infected macrophage unable to efficiently kill $M$. tuberculosis will inevitably die and the nature of this death can be of great importance for the subsequent course of infection. An aged, stressed or infected cell can undergo a number of different types of cell death where the major ones consist of apoptosis, necrosis and pyroptosis. These have all been implicated in macrophages during infection with $M$. tuberculosis. Apoptosis and necrosis will be discussed in this chapter, whereas inflammasomerelated cell death, such as pyroptosis, will be described in later chapters.

Apoptosis is an important part of the development and homeostasis of human tissues and serves as an immunologically silent way for cells to die. Apoptosis is recognized by a number of morphological and biochemical features including cell shrinkage, chromatin condensation, DNA fragmentation, permeabilization of the outer mitochondrial membrane and fragmentation of the cell into smaller apoptotic bodies with intact plasma membrane. Apoptotic bodies are then quickly phagocytosed by surrounding cells. If surrounding phagocytes fail to do so, the apoptotic bodies will eventually undergo post-apoptotic necrosis and disintegrate, spilling their contents into 
the extracellular milieu (209). Apoptosis is controlled by two major protein families; the Bcl-2 family and a family of aspartate-specific proteases called caspases, where the caspases are usually divided into upstream initiator caspases (caspase-2, -8, -9 and -10) and downstream executioner caspases (caspase-3, -6 and -7). Apoptosis is induced by a range of stimuli, which will activate one of two major apoptotic pathways, the intrinsic and extrinsic pathway. The extrinsic pathway is activated by the stimulation of death receptors on the surface of the cell, such as TNF receptor or Fas. In addition to NF- $\mathrm{kB}$ activation and survival signals, activation of the TNF receptor can induce the formation of a death-inducing signaling complex (DISC), a platform for recruitment and activation of the initiator caspases; caspase-8 and -10. The initiator caspases subsequently cleave the executioner caspases, which in turn cleave a large set of substrates leading to characteristic morphological and biochemical hallmarks of apoptosis (210). The intrinsic pathway is activated by a number of cellular stresses such as DNA damage, nitric oxide and ROS and is regulated by the Bcl-2 family. The members can be grouped into three functional subgroups that either inhibit apoptosis (including Bcl-2 and Bcl-XL), promote apoptosis (Bax, Bak and Bok) or regulate the activity of the other $\mathrm{Bcl}-2$ proteins. Under normal conditions, the anti-apoptotic members directly inhibit the activation of the proapoptotic Bcl-2 proteins, while the third subgroup has been proposed to either stimulate the activation of the proapoptic members or inhibit the function of the anti-apoptotic Bcl-2 proteins. When apoptosis is induced, the propaptotic $\mathrm{Bcl}-2$ members will become activated and stimulate permeabilization of the outer mitochondrial membrane and release of cytochrome c into the cytosol. Cytochrome c forms a complex termed the apoptosome together with the Apaf- 1 protein which recruits and activates the initiator caspase-9. Activated caspase- 9 in turns activates the executioner caspases and the apoptotic program is carried out (211). The intrinsic and extrinsic pathways are tightly connected and crosstalk occurs at the level of the mitochondria. For example capase-8, which is a part of the extrinsic pathway, can in certain cells types cleave the cytosolic protein Bid, which in turn activates the proapoptotic members of the Bcl-2 family in the mitochondria (212).

Necrosis, on the other hand, is a type of cell death characterized by cellular and organelle swelling, loss of plasma membrane integrity, no cleavage of apoptotic caspases and the release of intracellular DAMPs into the extracellular milieu, thereby acting as proinflammatory cell death. Necrotic cells are taken up in a slower and less efficient manner than apoptotic cells via macropinocytosis (210). Necrosis occurs under non-physiological stress such as heat, osmotic shock and addition of detergents. It has traditionally been viewed as accidental and uncontrolled, but in recent years studies have shown that necrosis can under certain conditions be orchestrated by the cell, a process known as necroptosis. Central to the process of necroptosis are two kinases, RIP1 and RIP3 that act as the initiators of the process. During TNF receptor 
signaling, phosphorylation of RIP1 and RIP3 can lead to necroptosis through an alternative induction of DISC, instead forming a necrosome. TLR signaling can also induce necroptosis through RIP1 and RIP3. Most studies have shown caspase-8 deletion or inhibition to be a prerequisite for the induction of necroptosis, suggesting that the enzyme acts to inhibit the pronecroptotic functions of RIP1 and RIP3. It is still not entirely clear if necroptosis releases specific DAMPs and induces a different immune response than accidental necrosis and its physiological role in bacterial infections needs further studies (213-214).

Cell death during infection by $M$. tuberculosis has been somewhat confusing due to the fact that the bacterium has been reported to induce both apoptosis (215-216) and necrosis (217-218). The nature of cell death induced is probably dependent on a number of factors such as the amount of bacteria, the particular cell type used and the analyzed time points. Analysis of cell death is also complicated by the fact that apoptosis and necrosis can share some features such as affected mitochondria and DNA damage (210). Finally, apoptosis and necrosis might not be mutually exclusive and apoptotic bodies will progress to post-apoptotic necrosis if not properly phagocytosed. However, comparisons between virulent and avirulent strains of mycobacteria have shown that induction of apoptosis is negatively correlated with virulence $(215,219)$ and that apoptosis is beneficial for the host by reducing bacterial numbers in infected cell cultures (220-221). In contrast to apoptosis, necrosis promotes bacterial replication and spreading to neighboring cells (37). Central necrosis of the granuloma is a hallmark of human tuberculosis and signifies disease progression and active tuberculosis (52). Furthermore, expression profile analysis of human wholeblood from tuberculosis patients and latently infected individuals show that elevated expression of genes related to apoptosis is important for control of latent tuberculosis (222). In light of this, it is commonly believed that virulent mycobacteria actively inhibits apoptosis and skews cell death towards necrosis. Inhibition of apoptosis is achieved by several mycobacterial factors, e.g. inhibiting the expression or secretion of mycobacterial superoxide dismutase, which neutralizes ROS, increases induction of macrophage apoptosis and lower bacterial burdens in vivo (223-224). In addition, the mycobacterial protein nuoG, a subunit of the type I NADH dehydrogenase involved in energy generation, also inhibits apoptosis. Deletion of the nuo $G$ gene leads to higher apoptosis in human macrophages and lower bacterial burden and increased host survival in mice (225). Though nuoG is not secreted from the bacterium, it acts in a similar manner as superoxide dismutase by neutralizing phagosomal ROS as well as decreasing the production of TNF from the macrophage (122). Inhibition of apoptosis is further mediated by bacterial cleavage of the host protein annexin-1, crucial for the terminal step of apoptosis when the apoptotic bodies are formed. Cleavage of annexin1 , which instead stimulates necrosis, is a unique ability of virulent M. tuberculosis (226). Virulent M. tuberculosis also influences the production of eicosanoids, small 
lipid mediators acting as short-range regulators of inflammation, in human macrophages. The bacterium increases the production of anti-inflammatory lipoxin $\mathrm{A}_{4}$ $\left(\mathrm{LXA}_{4}\right)$ which in turn inhibits production of prostaglandin $\mathrm{E}_{2}\left(\mathrm{PGE}_{2}\right)$. PGE $\mathrm{PG}_{2}$ normally exerts a protective effect on host mitochondria through predominantly inducing apoptosis while the loss of this eicosanoid stimulates necrosis by destabilizing the inner mitochondrial membrane (227). Deletion of $\mathrm{PGE}_{2}$ production in infected mice leads to higher bacterial burden, while deletion of $\mathrm{LXA}_{4}$ production instead generates higher levels of IL-12, IFN- $\gamma$ and iNOS, and leads to a lower bacterial burden (227228). M. tuberculosis does not only inhibit apoptosis but also actively promotes necrosis. Induction of necrosis in macrophages requires a functioning ESX-1 system and secretion of ESAT-6 $(37-38,184)$. The exact mechanism in which ESAT-6 induces necrosis is still not clear, but as previously mentioned, ESAT-6 from $M$. tuberculosis has been shown to dissociate from its partner CFP-10 and instead associate to phagosomal membranes (229) and displays membrane-lysing features (186), while ESAT-6 from $M$. marinum has been shown to form pores in the membrane of the host cell (184).

In summary, it has become clear that modulation of host cell death in macrophages is a potent immune evasion strategy of $M$. tuberculosis. It inhibits ROS-induced apoptosis, formation of apoptotic bodies and inhibits the protective functions of $\mathrm{PGE}_{2}$ while simultaneously promoting necrosis through ESAT-6 secretion. Inhibiting apoptosis in concert with phagosomal maturation block would be beneficial for the bacterium early during the infection of the macrophage, allowing it to replicate and adapt. When conditions are right or intracellular bacterial numbers reach a certain threshold, induction of necrosis is initiated by the bacteria, escaping the cell and infecting new ones.

\section{Efferocytosis}

Irrespective of the cell death mode induced in an individual cell upon mycobacterial infection, the dead cells need to be taken care of by other phagocytes to limit tissue damage. The antimicrobial effects of apoptosis are well documented, but a direct killing mechanism of apoptosis has still not been identified. Instead, the subsequent fate of the apoptotic body might be the crucial step.

Most macrophages undergo necrosis, but depending on the number of bacteria used for experimental infection a fraction will be able to induce apoptosis (230). In addition, the site of infection at early stages does not only include resident macrophages but also recruited neutrophils. Neutrophils are short-lived professional phagocytes specialized at ingesting pathogens, such as $M$. tuberculosis. Our understanding of the role of neutrophils in tuberculosis is relatively poor, but they are believed to be important early on during infection, while contributing to immunopathology at later time points. 
Studies on the ability of neutrophils to kill mycobacteria have generated varying results, but most of them support the idea that oxygen-independent mechanisms such as antimicrobial peptides from neutrophils can limit bacterial growth (231). However, unlike macrophages, neutrophils are prone to undergo apoptosis upon infection with wild type M. tuberculosis expressing the $19 \mathrm{kDa}$ lipoprotein (232-233). Subsequent uptake of apoptotic neutrophils or macrophages by other phagocytes, primarily macrophages, plays a role in infections and helps modulate the immune response. This process has been termed efferocytosis.

Apoptotic cells display different markers that enable their recognition and uptake. The binding of apoptotic cells is mediated by several different receptors that facilitate the uptake of the apoptotic cell, where most of them recognize phosphatidylserine (PS) exposed on the outside of the cell. The uptake of the apoptotic cell leads to the formation of a phagosome which will eventually fuse with lysosomes (234). The field of efferocytosis has been dominated by the view that uptake of apoptotic cells strictly induces an anti-inflammatory response in macrophages (235). This response would serve to limit tissue damage, but might also accidentally downregulate important antibacterial functions. However, uptake of apoptotic neutrophils during infection by M. tuberculosis instead promotes a proinflammatory response in macrophages (236237) as well as promoting bacterial killing by delivering antimicrobial peptides to the macrophage (50). Inversely, efferocytosis of dead macrophages by recruited neutrophils in M. marinum-infected zebrafish leads to ROS-mediated killing by the neutrophils, showing that efferocytosis is not a specific mechanism unique for macrophages (238). Efferocytosis of apoptotic macrophages by non-infected macrophages also controls bacterial growth both in vitro and in vivo (230), while efferocytosis of infected macrophages that display PS but have undergone more necrotic-like cell death does not have any bactericidal effects (239). Furthermore, the phagosome containing the $M$. tuberculosis-carrying apoptotic body is more efficiently acidified and spacious than the phagosome containing directly phagocytosed $M$. tuberculosis (230). Like with autophagy, this spacious phagosome with the bacterium encapsulated by an additional membrane derived from the apoptotic cell would inhibit the ability of the bacterium to efficiently block phagosomal maturation by simply increasing the distance between the bacterium and phagosomal membrane (208).

The early stages of infection are dominated by the presence of apoptotic cells which are taken up by surrounding macrophages, whether it is the abundant numbers of apoptotic neutrophils or the occasional apoptotic macrophage. It is the uptake of these apoptotic cells by surrounding cells rather than apoptosis per se that confer the beneficial effect of apoptosis in tuberculosis by activating macrophages, enabling more efficient phagolysosomal fusion and bestowing the macrophage with additional antimicrobial functions. The number of studies specifically focusing on efferocytosis 
is still rather few, but efferocytosis is emerging a major factor for control of bacterial growth and changes the view on many studies addressing the role of apoptosis in tuberculosis.

\section{Antigen presentation}

As resident alveolar macrophages try to restrict the early infection and limit bacterial growth, some macrophages and the professional antigen-presenting DCs will migrate from the lungs to local lymph nodes where they will present antigens to $\mathrm{T}$ cells via the major histocompatibility complex (MHC) class II pathway and initiate adaptive immune responses. Adaptive immunity against tuberculosis includes both CD4+ and CD8+ T cells, which produce IFN- $\gamma$. CD8+ T cells are also capable of recognizing a limited number of antigens presented on MHC class I molecules in infected macrophages, which are then killed through perforin and granulysin produced by the CD8+ $\mathrm{T}$ cell. Activation and expansion of $\mathrm{T}$ cells lead to an influx of these cells to the site of infection, where they exert their function by recognizing their specific antigen presented on MHC-expressing macrophages. Loss of either CD4+ or CD8+ T cells leads to hypersusceptibility to tuberculosis in experimental mice studies and in humans suffering from HIV/AIDS $(127,240)$.

To modulate or suppress the important effects of the adaptive immune response, many pathogens have evolved ways to interfere with antigen-presentation and function of antigen-presenting cells (APCs). Several strategies can be used including downregulation of antigen-presenting molecules such as MHC I/II, inhibiting migration of APCs, interfering with antigen processing and constantly changing the expression of antigenic proteins. $M$. tuberculosis can inhibit the expression of MHC class II and antigen processing in APCs through cell wall components such as the 19 $\mathrm{kDa}$ lipoprotein binding to TLR2. This inhibition persists even under IFN- $\gamma$ stimulation, which under normal conditions upregulates MHC expression (129, 241242). Decreased MHC class II in infected macrophages is mediated through suppression of class II transactivator (CIITA) expression, which is the transcription factor for MHC class II (243). APCs in mice lacking CIITA fail to present antigens and activate CD4+ T cells, which leaves them highly susceptible to tuberculosis (244). Similar TLR2-dependent inhibition of antigen presentation has been shown for the CD1 pathway, which is responsible for the presentation of lipid antigens such as LAM to T cells (245-246). Mycobacterial antigens are also presented on MHC class I, which present cytosolic self and non-self peptides, thereby alerting the immune system of intracellular cytosolic infection. Mice unable to present antigens on MHC I have increased susceptibility to tuberculosis (247). The question of how mycobacterial antigens are presented through the MHC class I pathway is still controversial, whether it is a matter of cross-presentation of antigens from the phagosome to MHC class I or 
translocation of antigens and bacterium to the cytosol $(187,248)$. Other routes, such as efferocytosis-induced presentation on MHC class I has also been suggested (249). Modulation of antigen presentation is also coupled to many of the other virulence traits of $M$. tuberculosis. In addition to limiting antibacterial function, a consequence of inhibiting phagosomal maturation is decreased antigen presentation (250). Inhibition of apoptosis and efferocytosis also has an impact on antigen presentation and initiation of adaptive immunity. M. tuberculosis infecting DCs suppress their migration to lymph nodes and delays the activation of CD4+ T cells. Migration of DCs and activation of CD4+T cells are restored if the bacterium is taken up via efferocytosis of apoptotic neutrophils or if apoptosis is enhanced by infection with a mutant bacterium lacking nuoG (251-252).

Antigen presentations together with soluble signals such as cytokines are the components that link the innate immunity to the adaptive and are a prerequisite for the induction of protective $\mathrm{T}$ cells. However, though adaptive immune responses have a crucial role in tuberculosis, the fact that $\mathrm{T}$ cell epitopes are hyperconserved implies that the bacterium relies on antigen-presentation and adaptive immunity as a part of its pathogenesis (20).

\section{The inflammasome}

There are numerous effector molecules and cellular pathways important for combating intracellular infections and many are absolutely crucial for mounting an efficient immune response. This chapter will focus on the functions of one particular pathway responsible for the generation of proinflammatory cytokines and induction of cell death. Central to this pathway is the multimeric protein platform referred to as the inflammasome. The assembly and function of the inflammasome itself as well as the effects of the cytokines produced upon inflammasome activation will be reviewed. Furthermore, the role of inflammasomes during tuberculosis will be discussed in the final section of the chapter.

\section{Structure of the inflammasome}

The basic inflammasome consists of an intracellular receptor, which initiates the assembly of the inflammasome upon a specific signaling event, a potential adaptor protein and the cystein protease caspase- 1 . Several copies of this basic unit are joined together to form a functional inflammasome. To date, four different receptors capable of initiating inflammasome assembly have been identified. Three are members of the NLR family (NLRP1b, NLRP3 and NLRC4) and one is the AIM2 protein, which is 
part of another protein family termed PYHIN. NLRP1b mainly recognize LT (113), while NLRC4 responds to bacterial flagellin (253). The most recently discovered inflammasome, containing AIM2, assembles in response to cytoplasmic DNA (254). The fourth inflammasome, containing NLRP3, is the most extensively studied and is, as mentioned earlier, promiscuous and reacts to a number of exogenous PAMPs and irritants as well as to endogenous DAMPs (116). NLRP3 is the NLR that has received most attention in the context of tuberculosis. NLRP3, upon activation, starts to oligomerize while recruiting an adaptor protein called apoptosis-associated speck-like protein containing a CARD (ASC) via its N-terminal PYD domain. ASC in turn enables the recruitment of the inflammatory caspase-1, which binds to the C-terminal CARD domain of ASC through its own CARD domain. ASC thereby acts as a molecular bridge between NLRP3 and caspase-1. Caspase-1 is recruited as an inactive zymogen, procaspase- 1 , and binding to an oligomerized inflammasome leads to the formation of procaspase- 1 dimers. As a dimer, the procaspase- 1 is cleaved by autoproteolysis into a large and small subunit termed p20 and p10, respectively, and thus becomes activated. Active caspase- 1 subsequently induce cell death or process members of the IL-1 family, IL- $1 \beta$ and IL-18, from their pro-forms to active cytokines (255).

\section{Activation of the inflammasome}

Activation of the inflammasome and subsequent production of IL-1 $\beta$ from its $31-\mathrm{kDa}$ proform to the $17-\mathrm{kDa}$ mature form requires two distinct signals in macrophages. The first signal is described as a priming step necessary to induce the transcription of proIL-1 $\beta$ while the second step is the inflammasome assembly and activation of caspase-1. Transcription of proIL-1 $\beta$ is dependent on NF- $\kappa B$ activation, which can be induced in a number of ways including TLR signaling (256). This priming step was first believed to be required solely for inducing upregulation of proIL- $1 \beta$, but NF- $\kappa B$ simultaneously upregulates the transcription of NLRP3, which is also a prerequisite for inflammasome activation and production of mature IL-1 $\beta$ (257). The exact nature of the second signal, which is thought to interact with the ligand-binding LRR domain of NLRP3 and required for activation of the NLRP3 inflammasome, has however remained elusive for over a decade, largely due to the fact of the structural and functional diversity among the NLRP3 activators.

Three major pathways of inflammasome activation have so far been suggested. The first pathway involves the activation of the NLRP3 inflammasome by ATP stimulation or pore-forming toxins. Stimulating inflammasome assembly in macrophages with ATP is via binding of ATP to the P2X7 receptor, which acts as a nonselective cation channel. The activation of the P2X7 receptor, or addition of the ionophore nigericin, leads to efflux of potassium from the cell (258), which in turn is required for the 
activation of the NLRP3 inflammasome (259). The role of potassium efflux during inflammasome activation has been strengthened by subsequent studies showing that potassium efflux is induced in murine macrophages by most NLRP3 activators (260). ATP-driven P2X7 activation and subsequent potassium efflux was first shown to induce the formation of large pores in the plasma membrane, leading to the idea that inflammasome-activating PAMPs and DAMPs gains access to the cytosol and directly interacts with NLRP3 (261-262). However, the role of these large pores in inflammasome activation has later been shown to be redundant for inflammasome activation (263). Though some NLRP3 agonist are able to enter the cytosol, thus being able to directly bind to NLRP3, the fact remains that the stimuli capable of activating NLRP3 are very heterogeneous, making a direct interaction between the different activators and NLRP3 very unlikely $(101,264)$.

A second pathway shared by several NLRP3 activators involves lysosomal damage or rupture. This pathway is mainly implicated in inflammasome activation by crystalline substances, like exogenous silica, asbestos and alum (265-266) or endogenous uric acid crystals (267). Alum, silica and uric acid crystals are all phagocytosed and subsequently cause lysosomal damage, leading to the introduction of lysosomal contents in the cytosol, thereby activating NLRP3 (266, 268). In fact, artificial disruption of lysosomes in the absence of crystals can activate the NLRP3 inflammasome. Based on inhibition experiments, it has been suggested that the mediator of inflammasome activation leading to production of IL- $1 \beta$ is lysosomal cathepsin B (266, 269-270). The prerequisite for cathepsin B in inflammasome activation has however been questioned due to the observations that no effect on IL-1 $\beta$ production is seen after inhibiting lysosomal acidification required for cathepsin B activity or from cells deficient in cathepsin B (271). Irrespective of the mediator, lysosomal destabilization readily induces inflammasome activation, but since this pathway seems to be limited to particulate NLRP3 activators that induce lysosomal damage, it probably represents an upstream event of a common pathway shared by all NLRP3 activators.

The third pathway is ROS-dependent activation of the NLRP3 inflammasome. ROS seem to be produced prior to inflammasome activation irrespective of the stimuli and ROS scavengers are able to block activation of the inflammasome induced by both ATP and crystalline particles (265, 272-273). Activation of NOX2 and a subsequent oxidative burst is an important source of ROS in macrophages and initial studies using an shRNA approach reported a decreased production of IL-1 $\beta$ upon silencing a subunit of NOX2 (265). However, genetic knock-out of the NOX2 system does not decrease activation of NALP3 inflammasome by silica crystals, uric acid crystals or ATP (266). Neither is the IL- $1 \beta$ production affected upon stimulation of silica or uric acid crystals in mononuclear cells from patients suffering from chronic granulomatous disease, a 
disorder caused by the lack of functional NOX2 (274). Instead, the ROS required for inflammasome activation is thought to be derived from the mitochondria. The involvement of the mitochondria in inflammasome activation is supported by several observations; the potassium efflux also required for inflammasome activation is known to be coupled to mitochondrial ROS generation (275) and the induction of mitochondrial ROS production leads to NLRP3-dependent IL-1 $\beta$ secretion, which can be blocked by a ROS inhibitor or by inhibiting mitochondrial metabolic activity (276). Furthermore, inhibiting the clearance of damaged and ROS-producing mitochondria by autophagy enhances inflammasome activation by increasing the levels of intracellular ROS (276-278). ROS production and mitochondrial damage lead to the translocation of mitochondrial components such as oxidized mitochondrial DNA into the cytosol, which can activate the NLRP3 inflammasome, caspase- 1 and increase IL$1 \beta$ production $(277,279)$. The interplay between mitochondrial ROS production and NLRP3 activation is further highlighted by the fact that the thioredoxin (TRX)interacting protein (TXNIP), which under normal conditions is bound to the endogenous antioxidant TRX, dissociates from TRX following increased mitochondrial ROS production and instead interacts with NLRP3 (280). Loss of TXNIP leads to decreased caspase- 1 activity and IL- $1 \beta$ production, while knockdown of TRX, the endogenous binding partner of TXNIP, increases IL- $1 \beta$ production in response to NLRP3 activators $(265,280)$. However, the prerequisite for ROS and the involvement of the mitochondria has been recently questioned by a study showing that potassium efflux activates the NLRP3 inflammasome independently of ROS production and mitochondrial damage, thereby favoring potassium efflux as a common denominator of inflammasome activation (260). Furthermore, it has been suggested that mitochondrial ROS is required for the priming step and induction of NLRP3 expression, rather than the actual activation (281). 


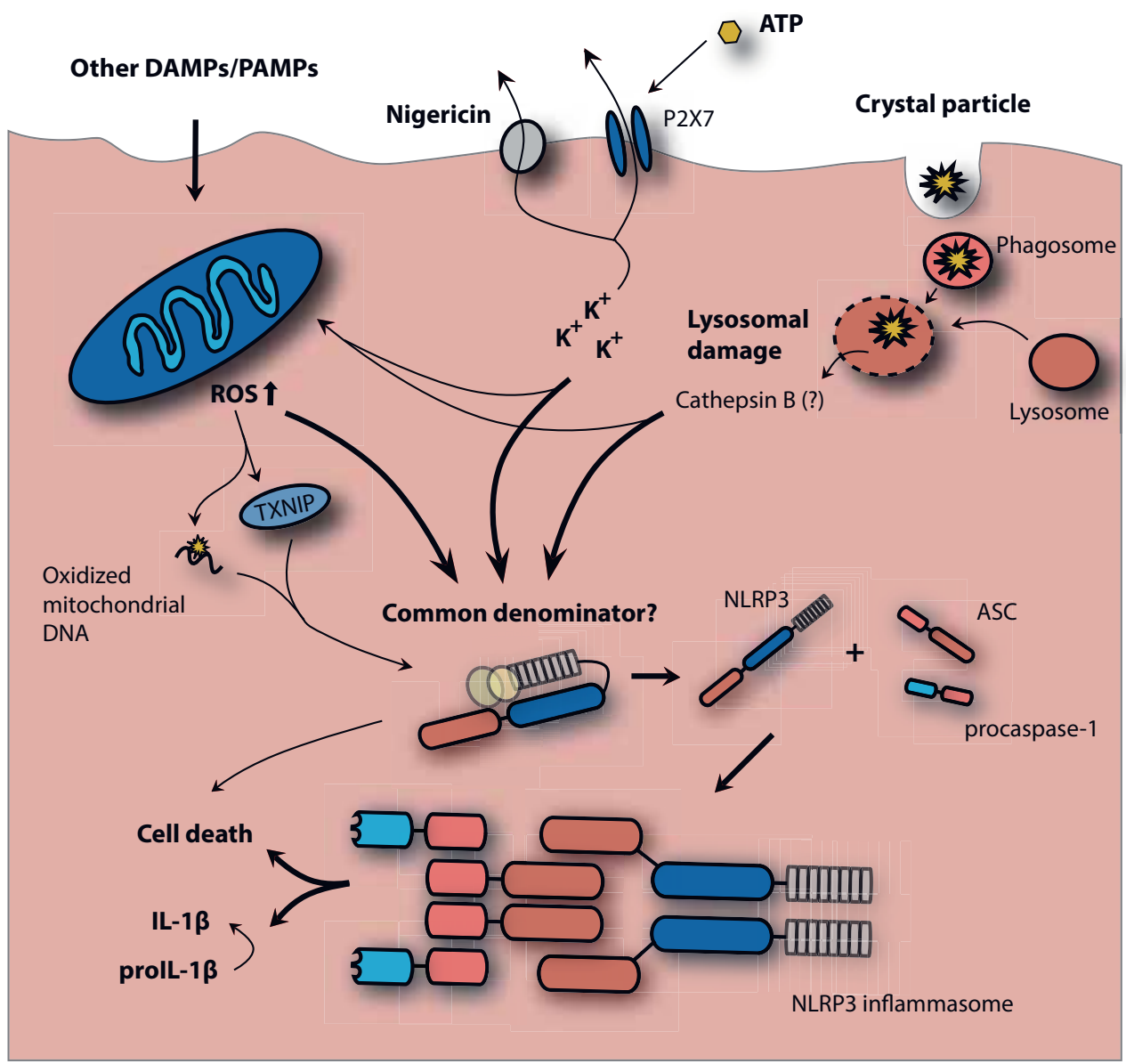

Figure 5. The activation of NLRP3 and assembly of the inflammasome can be achieved through three major pathways. Lysosomal damage following phagocytosis of crystal particles leads to NLRP3 activation, possibly mediated by lysosomal cathepsin B. The second pathway is exemplified by stimulation with ATP or nigericin, which induce an efflux of potassium, through the $\mathrm{P} 2 \mathrm{X} 7$ receptor or by directly forming a pore in the membrane. The third pathway proposes mitochondrial ROS as the mediator for NLRP3 activation. This ROS has been suggested to lead to activation of the protein TXNIP or release of oxidized DNA from the mitochondria, thereby activating NLRP3. Studies have implicated both potassium efflux and mitochondrial ROS as the least common denominator, suggesting a cross-talk between the pathways. Irrespective of the stimuli, activated NLRP3 oligomerizes and recruits the adaptor protein ASC, which in turn recruits procaspase-1. Two closely situated procaspase-1 molecules are then thought to be activated through autoproteolysis. Actived caspase-1 cleaves the pro-form of $I L-1 \beta$ (and $I L-18$ ) to its active form and can induce a certain type of cell death, termed pyroptosis. Activation of NLRP3 can also lead to cell death in a caspase-1indpendent manner, which is usually referred to as pyronecrosis. 
The mechanism responsible for the activation of the NLRP3 inflammasome is still not fully understood and the literature supports both mitochondrial dysfunction with ROS production and potassium efflux as common denominators for the plethora of molecules able to activate NLRP3. Though these pathways might be functionally separate with one being a persistent epiphenomenon during experimental activation of NLRP3, our current knowledge rather suggests that both pathways are required for an optimal inflammasome-dependent response and this response likely includes a high level of cross-talk between the two pathways. Irrespective of the activating stimuli and downstream signaling pathway, the signals must ultimately be recognized by NLRP3. This is likely to be a rather specific recognition via the LRR domain, which acts as the specific ligand-binding domain in TLRs. The promiscuous nature of NLRP3 and different requirements for potassium efflux, ROS production or other signals in inflammasome activation in different studies could be explained by induction a so far unidentified endogenous ligand that binds and activates NLRP3. This would resemble the role of the endogenous Spatzle protein in Drosophila melanogaster, which upon proteolytical processing is responsible for activation of Drosophila Toll receptors (282). A hypothetical model of inflammasome activation is shown in figure 5.

\section{Genetic alterations of the inflammasome}

In addition to both endogenous and exogenous molecules capable of activating NLRP3, many mutations and polymorphisms in the NLRP3 gene lead to a constitutively active inflammasome or a lower threshold for inflammasome activation. Genetic alterations in NLRP3 are associated with increased susceptibility to a number of inflammatory diseases including gout, psoriasis, rheumatoid arthritis and Crohn's disease (283-286). However, the association between NLRP3 alterations and inflammatory disease caused by excessive IL-1 $\beta$ is strongest in a group of autoinflammatory disorders known as cryopyrin-associated periodic syndromes (CAPS) and includes familial cold autoinflammatory syndrome, Muckle-Wells syndrome and chronic infantile neurologic cutaneous articular syndrome. Though they differ in clinical severity, these syndromes are characterized by recurrent fever, rashes and joint inflammation. Sixty percent of all CAPS patients have known mutations in $N L R P 3$ and they usually respond well to anti-IL-1 $\beta$ treatment, such as the IL-1R antagonist anakinra (287). To date, there are more than 150 reported sequence variants for NLRP3 with varying degree of penetrance, frequency and disease severity, and several are present in healthy individuals as well. Interestingly, an overwhelming majority of the disease-causing mutations are found in the NACHT domain of NLRP3 (288) and has led to the hypothesis that these mutations inhibit the interaction between the NACHT and LRR domains of NLRP3, thereby disrupting the suppressive conformation of the whole protein. Though it has not been experimentally confirmed, 
this is thought to lead to spontaneous oligomerization or a lower threshold for activation of the NLRP3 inflammasome (289-291).

\section{Regulation of the inflammasome and IL-1 $\beta$ production}

The release of proinflammatory cytokines like IL-1 $\beta$ is crucial for a proper immune response, but could also become detrimental to the host if not properly regulated. The NLRP3 inflammasome can be regulated in a number of ways to limit or enhance IL-1 $\beta$ production. These regulatory mechanisms include different types of signals, interaction with other proteins and transcriptional regulation. Since the generation of mature IL-1 $\beta$ requires both induction of proIL-1 $\beta$ and NLRP3 expression as well as activation of the inflammasome, regulation of IL-1 $\beta$ processing occurs on both a transcriptional and post-transcriptional level. Transcription of proIL-1 $\beta$ in macrophages is upregulated by most proinflammatory signals but can also be downregulated following direct stimulation by the anti-inflammatory cytokine IL-10 or indirectly through signals that induce production of IL-10, such as type I interferons (292-293). Transcriptional regulation of NLRP3 is affected by the same stimuli as proIL-1 $\beta$, but is further regulated by the short half-life of NLRP3 mRNA and microRNA-induced suppression of NLRP3 by miRNA-223. Through complementary binding, miRNA-223 lowers the levels of NLRP3 mRNA, leading to decreased protein expression and IL-1 $\beta$ production (294). Post-transcriptional regulation of both IL-1 $\beta$ processing and the inflammasome also occurs in several ways. Autophagy, in addition to its role in inflammasome activation by clearance of damaged mitochondria, can regulate both IL-1 $\beta$ levels and inflammasome activation. Induction of autophagy in murine macrophages leads to decreased levels of proIL-1 $\beta$ after LPS stimulation and intracellular IL-1 $\beta$ co-localizes with LC3-positive autophagosomes (295). Activation of the inflammasome in human macrophages simultaneously induces autophagy, which leads to co-localization of activated inflammasomes to LC3-positive autophagosomes (296). In addition, autophagy can also limit other functions of the inflammasome, such as induction of cell death (297). Other regulatory mechanisms of inflammasome activation include inhibitory nitrosylation of NLRP3 by NO upon IFN$\gamma$ stimulation and decreased NLRP3 activation following type I interferon signaling (293, 298). The assembly of the different components of the inflammasome can also be regulated by two different families of small proteins; CARD-only proteins (COPs) and Pyrin-only proteins (POPs). Both these two families exert their function by acting as "decoys". For example, the CARD domain of the COPs ICEBERG and pseudo-ICE share a high degree of homology with the CARD domain of caspase- 1 and can therefore sequester caspase-1, making it unavailable for activation by the inflammasome (299). Over-expression of ICEBERG and pseudo-ICE in THP-1 cells effectively reduces the release of IL-1 $\beta$ upon LPS stimulation (300). Similar to the COPs, the Pyrin domain of the POPs resembles the Pyrin domain of either ASC or the 
NLRs, thereby able to sequester or block the interaction between these inflammasome components. Many of these regulatory proteins lack homologues in mice, indicating that regulation of inflammasome activity in human cells is more complex (299). Another protein that has been implicated in regulation of the inflammasome and caspase- 1 is CARD8. It negatively regulates IL-1 $\beta$ in two different ways. First, CARD8 has the ability to directly interact with and inhibit the activity of caspase-1 and secondly, it can also inhibit NF- $\mathrm{kB}$ activation, thereby decreasing the formation of proIL-1 $\beta$ (301). CARD8 was originally described as a member of the NLRP3 inflammasome (114) but this has not been confirmed in subsequent studies. However, the link between CARD8 and NLRP3 is supported by the fact that polymorphisms in CARD8 together with NLRP3 polymorphisms have been implicated in several diseases including Crohn's disease, psoriasis and rheumatoid arthritis (284, 302-304). Lastly, there are also regulatory proteins that can promote inflammasome assembly or are required for NLRP3 activation. Examples include the previously mentioned chaperones HSP90 and SGT1, which are required for maintaining NLRP3 in a signaling-competent conformation (99) or the guanylate-binding protein 5 (GBP5), which enhances IL-1 $\beta$ processing by promoting inflammasome assembly upon activation by soluble and bacterial stimuli, but not crystalline activators, in human macrophages (305).

Though the macrophage can regulate the formation of mature IL-1 $\beta$ in numerous ways, inflammasome activation and caspase- 1 activity are in some cases redundant for the release of mature IL-1 $\beta$. For example, the requirement of a second signal specifically activating the inflammasome is not required in human monocytes. Instead these cells release IL-1 $\beta$ upon addition of LPS alone as a consequence of their ability to constitutively release ATP, which in turn leads to a constant activation of caspase-1. Unlike monocytes, monocyte-derived human macrophages or alveolar macrophages usually require both a priming step and subsequent activation of caspase- 1 through inflammasome activation (306). However, the direct release of IL-1 $\beta$ by LPS stimulation has been shown in human macrophages differentiated or stimulated with IFN- $\gamma$, suggesting that robust activation of macrophages can sensitize the cell to respond to additional stimuli and lower the threshold for inflammasome activation, a phenomenon which might be more pronounced in human cells (305-306). Processing of IL-1 $\beta$ can occur independently of caspase- 1 in murine DCs (307) and human neutrophils can induce extracellular cleavage of proIL-1 $\beta$ by proteases such as protease- 3 and elastase (308). Caspase-1-independent processing of IL-1 $\beta$ by caspase8 in parallel with inflammasome-driven IL-1 $\beta$ production can occur in murine macrophages, a process requiring the activation of RIP3 and subsequent ROS production, thereby linking necroptosis to IL- $1 \beta$ production (309). In line with this, caspase- 8 is able to cleave proIL-1 $\beta$ in vitro and caspase- 8 activation through TLR3 stimulation also lead to increased release of IL-1 $\beta$ from murine macrophages (310). 
There are many pathways and mediators that regulate inflammasome activation and IL-1 $\beta$ production and their role in disease is in many cases poorly understood. It is likely that these regulatory mechanisms and signals serve to fine-tune the responsiveness or activation threshold of NLRP3 as well as the availability of all the components necessary for IL-1 $\beta$ production to take place. Together, these signals ultimately decide the magnitude and timing of inflammasome and caspase- 1 activity. Though complex in nature, the number of regulatory mechanisms shows the central role of inflammasomes in inflammatory responses and the importance of controlling this response, while the fact that inflammasome-independent pathways of IL- $1 \beta$ processing exists, indicates that a complete loss of IL-1 $\beta$ production would be detrimental to the host.

\section{Inflammasome-related cell death}

In addition to the generation of mature IL-1 $\beta$ and IL-18 being well-established consequences of inflammasome activation and caspase- 1 activity, this pathway also has the potential to induce certain types of cell death, termed pyroptosis or pyronecrosis. In addition to the accompanying cytokine production, the necrotic nature of inflammasome-mediated cell death leads to spilling of intracellular content, making these types of cell death capable of eliciting a strong inflammatory response. Most of the studies on inflammasome-mediated cell death have been performed with infections using live bacteria and have revealed that the nature of the inflammasome-mediated cell death is largely dependent on the type of NLR that is activated (311).

Pyroptosis is dependent on caspase- 1 and displays features of both apoptosis and necrosis, but is distinct from both of these major death modalities. Pyroptosis has mainly been described in macrophages and is accompanied by the release of IL- $1 \beta$ and IL-18, but does occur independently of IL-1 $\beta$ or IL-18 signaling (311-312). In addition to being strictly dependent on caspase-1, pyroptosis is recognized by DNA fragmentation, usually thought to be an apoptotic feature, but without the chromatin condensation. Pyroptosis also displays necrotic features by the loss of plasma membrane integrity. Finally, in contrast to apoptosis, pyroptosis does not directly involve loss of mitochondrial membrane potential and mitochondrial dysfunction, but instead has been suggested to be dependent on the ATP generation of functional mitochondria (311-313). Pyroptotic cell death can be induced through the non-NLR AIM2 inflammasome by cytosolic DNA (314-315) or even by a so far unidentified pathway in murine cells acting through caspase-11, the homologue of human caspase4 and caspase-5 (316). However, pyroptosis with the characteristic features of cell death and a dependency on caspase-1, has been mainly found to be induced during macrophage infections with flagellin-expressing bacteria such as Legionella and Salmonella (317-320) or by adding LT to macrophages (319, 321). Not surprisingly, 
this induction of pyroptosis by the different stimuli is dependent on the activation of NLRC4 and NLRP1b respectively, and since both contain a CARD domain, induction of pyroptosis does not necessarily include ASC $(318,321)$. In line with the distinct roles of different NLRs in induction of pyroptosis, intracellular infection of murine macrophages lacking either caspase-1, NLRP3 or NLRC4 with the Gram-negative bacterium Burkholderia pseudomallei has shown that though caspase-1 is essential for both cytokine production and pyroptosis, the cytokine production is mainly dependent on NLRP3, upstream of caspase-1, while the signal for caspase-1-dependent pyroptosis seems to originate from NLRC4 (322). Cytokine production and pyroptosis as being two separate events are further strengthened by the fact that pyroptosis can be induced prior to an initial priming step by the NLRC4 inflammasome in murine macrophages. This "unprimed" pyroptosis occurs without the usual simultaneous release of cytokines, clearly separating the two established functions of the active inflammasome (313). So what decides if activation of these NLRs will induce cytokine production or lead to pyroptotic cell death? Induction of pyroptosis was first suggested to be a result of a single large ASC oligomer that forms in LPS-stimulated THP-1 cells overexpressing ASC and this large supramolecular complex was named "pyroptosome" (323). However, later studies showed that this large ASC focus is indeed formed upon inflammasome activation and is important for cytokine production, but induction of pyroptosis is not dependent on ASC but on CARDcontaining NLRs outside of the ASC focus that does not stimulate classic autoproteolysis of caspase-1. Caspase-1 instead remains in an unprocessed, alternatively activated form in these inflammasomes and is able to induce pyroptosis (324). The specific signals leading to the formation of these "pyroptotic" inflammasomes needs to be further elucidated and the physiological role of pyroptosis is still not entirely clear. Mice lacking caspase- 1 are more susceptible to certain bacterial infections and more resistant to sepsis, but the observed phenotype is not completely "rescued" upon inhibiting IL-1 $\beta$ and IL-18 production, implying that other caspase-1 functions, such as pyroptosis, play a role (325-326). It should be noted that caspase-1-deficient mice used in many studies so far are also unintentionally deficient in caspase-11, which is situated upstream of caspase-1, making it more difficult to draw conclusions on the role of caspase- 1 in vivo (316). A study directly addressing the role of pyroptosis in vivo has shown that pyroptosis can be an important protective mechanism during bacterial infections. Using a Salmonella strain with inducible flagellin expression, it was shown that induction of pyroptosis correlates with bacterial clearance in a manner independent of inflammasome-related cytokines (327). The beneficial effects of pyroptosis might include release of intracellular bacteria, which can then be phagocytosed and killed by other types of phagocytes like neutrophils (327), or the extracellular release of antimicrobial factors from lysosomes in cells undergoing pyroptosis (328). 
NLRP3-mediated, caspase-1-dependent pyroptosis has been shown in cell types other than macrophages (329) as well as in macrophages stimulated specifically with ATP (330-331). Although it is believed that NLRP3 also has the ability to induce pyroptosis, few studies have actually shown caspase-1-dependent induction of DNA fragmentation and pore formation upon NLRP3 activation in macrophages. Instead, several studies in both human and murine cells have shown that NLRP3-mediated cell death is more closely related to necrosis and the term pyronecrosis has been used to describe this phenomenon. Several pathogens including Shigella, Neisseria and Klebsiella have shown to induce pyronecrosis (269-270, 332). Furthermore, activating mutations in NLRP3 associated with CAPS also lead to pyronecrotic cell death (332334). Pyronecrosis is less well defined than pyroptosis, since morphological features seem to be limited to increased permeability of the plasma membrane, a feature also present during necrosis and pyroptosis (311). It does not include DNA fragmentation and is independent of caspase-1, while being reduced during silencing or knock-out of NLRP3 and ASC (269-270, 332-334). Pyronecrotic cell death can be inhibited by inhibiting cathepsin B, suggesting that NLRP3 activation following lysosomal damage is the NLRP3-activating pathway responsible for inducing pyronecrosis. It should however be noted that the effects of cathepsin B inhibition were all observed following addition of the inhibitor CA-074Me, which might be a consequence of off-target activity since other inhibitors of cathepsin B are less effective in inhibiting cell death (264, 335). As previously mentioned, the necroptosis-inducing RIP3 also activates NLRP3 (309), suggesting a cross-talk between inflammasome activation and induction of necroptosis.

Inflammasome-mediated cell death is an independent effector mechanism of inflammasome activation with rather drastic consequences and potent proinflammatory effects. Though inflammasome-mediated cell death is hard to dissect from the cytokine production induced by an active inflammasome, the different pathways regulating the balance between cell death and cytokine production as well as the relevance of these types of cell death are starting to emerge.

\section{Non-canonical functions of the inflammasome}

Though the production of IL-1 cytokines and inflammasome-mediated cell death have been the main focus of most studies on inflammasome function, inflammasome assembly and caspase-1 activation have additional functions. In addition to the IL-1 cytokines as substrates, active caspase- 1 in THP- 1 cells can cleave over 40 different substrates involved in many different cellular processes such as glycolysis, protein translation and the cytoskeletal machinery (336). Inflammasome activation and the enzymatic activity of caspase- 1 is not only crucial for cleaving IL- 1 cytokines to their active form, but also drives the actual secretion of both inflammasome-related 
cytokines and other leaderless cytokines like IL-1 $\alpha$ (337-338). Furthermore, activation of the inflammasome can lead to activation of other caspases such as caspase-8 (339) and caspase-7 (340), thus stimulating apoptosis. Finally, NLRP3, NLRC4 and ASC together with activated caspase- 1 are required for the activation of sterol regulatory element binding proteins (SREBPs), which are crucial transcription factors in lipogenesis and involved in membrane repair caused by bacterial pore-forming toxins, indicating that under certain conditions, inflammasome activation can also induce prosurvival signals (341).

The non-canonical functions of inflammasome activation, such as the induction of apoptosis and increased membrane repair, fits well with what is thought to be protective mechanisms important in combating tuberculosis, and studies on inflammasome functions that go beyond cytokine production would be of interest. The cleavage of caspase-7 and caspase-8 or counteracting the membrane-lysing activity of ESAT-6 might represent important effector mechanisms that complement the inflammasome-mediated production of cytokines and the large set of potential caspase- 1 substrates strongly suggests that caspase- 1 indeed has additional functions.

\section{Interleukin-1 $\beta$ and the IL-1 family}

Signaling transduction by the IL-1 family of cytokines consists of 11 ligands, including IL-1 $1 \beta$, IL-1 $\alpha$, IL-18 and IL-33 and 10 receptors with IL-1R type I (IL-1RI) and IL-1R type II (IL-1RII) being the most prominent members. The IL-1 family is sometimes referred to as innate cytokines since IL-1RI, like TLRs, consist of an intracellular TIR domain, signals through MyD88 and IRAKs and induce NF-kB activation. They are also different to most other cytokine families in that many of the IL-1 family members lack signal peptides required for secretion via the classical secretory pathway (308). Of the IL-1 family cytokines, IL-1 $\beta$ and IL-18 are both processed through activation of caspase- 1 by inflammasomes. Processing of the Th2polarizing cytokine IL-33 into a biologically active form was first thought to be dependent on inflammasome activation and caspase- 1 as well, but more recent studies have shown that caspase- 1 is not required for the production of active IL-33 (342). In fact, caspase-1 instead inactivates IL-33, leaving IL-33 unable to signal through its receptor (343). The following discussion will focus on IL-1 $\beta$, which is within the main scope of this thesis and will serve as the model member of the IL- 1 family, but it is important to remember that IL- $1 \beta$ production through inflammasome activation is tightly coupled to the production of mature IL-18, which can also have direct effects on macrophages and induce production of proinflammatory cytokines (344). Furthermore, one of the members of the IL- 1 family that is closely related to IL- $1 \beta$ is IL- $1 \alpha$. Though the biological function of IL- $1 \alpha$ and IL- $1 \beta$ differs and IL- $1 \alpha$ is 
expressed constitutively in a number of cell types, they both signal through IL-1RI and exhibit overlapping functions (308).

The biological functions of IL-1 $\beta$ are numerous and diverse. It is known as a pyrogen and induces fever at relatively low concentrations. On a systemic level, effects can also include loss of appetite, headache, decreased blood pressure, changes in insulin levels and production of acute phase proteins from the liver. Local effects of IL- $1 \beta$ include infiltration of immune cells like neutrophils, production of other interleukins, angiogenesis and upregulation of adhesion molecules. Many of these effects are mediated through induction of cyclooxygenase 2 (COX2) and subsequent production of prostaglandins, especially PGE $\mathrm{PG}_{2}$. In addition to direct effects, IL-1 $\beta$ also induces expression of many genes both directly involved in proinflammatory responses as well as growth factors, extracellular matrix proteins and proteins involved in tissue remodeling (345). Much of the regulation of IL-1 $\beta$ is on a transcriptional level and by regulating the activation of the inflammasome, as described in earlier sections. However, to avoid excessive inflammation and tissue damage, signaling by IL- $1 \beta$ is also tightly regulated by mechanisms downstream of inflammasome activation and processing of proIL-1 $\beta$. The fact that mature IL-1 $\beta$ lacks a signal peptide and can be secreted in a Golgi-independent manner might constitute an initial regulatory step after processing by caspase-1. IL-1 $\beta$ co-localizes with autophagosomes (295), but can also co-localize with secretory lysosomes, which release mature IL-1 $\beta$ together with inflammasome components into the extracellular space (346). This suggests that activated inflammasomes producing mature IL- $1 \beta$ rapidly localize to lysosomes, which can regulate the release of IL- $1 \beta$ by retaining and degrading the cargo or be shuttled to the plasma membrane for secretion. After production and release, IL-1 $\beta$ is further regulated in several ways. Binding of IL- $1 \beta$ to IL-1RI can be inhibited by the endogenous IL-1 receptor antagonist (IL-1Ra), which is produced constitutively in non-stimulated macrophages differentiated in vitro (347). IL-1Ra is also produced as a hepatic acute phase protein, and elevated levels of IL-1Ra can usually be seen following a plasma increase of IL-1 $\beta$ (348). Soluble IL-1Ra does not act through inhibitory signals but functions purely as a receptor antagonist where concentrations $10^{6}$ times greater than that of IL- $1 \beta$ fail to elicit any responses in humans. Recombinant IL-1Ra (anakinra) is therapeutically used in a number of inflammatory diseases (308). Furthermore, the inhibitory effect of IL-1Ra is further enhanced by the fact that the receptor IL-1RI has greater affinity for IL-1Ra than IL-1 $\beta$. Strong binding of IL-1 $\beta$ to IL-1RI also requires a co-receptor, IL-1R accessory protein (IL-1RAcP), which is redundant for binding both IL- $1 \alpha$ and IL-1Ra (349). In contrast, IL-1 $\beta$ instead has higher affinity to the decoy receptor IL-1RII than IL-1 $\alpha$ and IL-1Ra. IL-1RII lacks a cytoplasmic signaling domain, thus binding IL-1 $\beta$ without eliciting a signal (350351). IL-1RII can also recruit IL-1RAcP, limiting the availability of IL-1RAcP to form a functional complex with IL-1RI and IL-1 $\beta$ (352). Finally, both IL-1 receptors as well 
as IL-1RAcP also exist in a soluble form, thereby sequestering IL-1 $\beta$ and limiting its effects on cells and tissues (351).

When combining the regulatory mechanisms that exist to control the activation of the inflammasome and production of mature IL- $1 \beta$ with the additional mechanisms that control signaling by IL- $1 \beta$ through its receptor, an extremely complicated network of checkpoints and inhibitory signals emerge. The multifunctional nature of IL- $1 \beta$ makes it a crucial cytokine for almost any kind of inflammatory response. It is this very same multifunctional and potent nature that requires rigorous control and as shown for numerous inflammatory diseases, failure to do so can have detrimental effects for the host.

\section{Interleukin-1 $\beta$ and inflammasome activation in tuberculosis}

Virulent $M$. tuberculosis activates the NLRP3 inflammasome through expression of ESAT-6. Purified ESAT-6 can directly activate caspase-1 in THP-1 cells and loss of ESAT-6 severely compromises the induction of IL-1 $\beta$ by the bacteria (353). Likewise, infection of macrophages lacking NLRP3, the adaptor protein ASC or caspase-1 with virulent wild type $M$. tuberculosis is also characterized by low levels of IL-1 $\beta$ (354356), showing that in macrophages in vitro, the NLRP3 inflammasome is activated in an ESAT-6-dependent manner and is responsible for the production of IL- $1 \beta$. Furthermore, M. tuberculosis can also inhibit the NLRC4 inflammasome through the expression of the metalloprotease Zmp1 (167).

The IL-1 $\beta$ produced by the NLRP3 inflammasome upon $M$. tuberculosis infection has several effects and IL-1 $\beta$, together with vitamin $\mathrm{D}$, leads to the expression of antimicrobial peptides in human monocytes, which enhances monocyte killing of the attenuated H37Ra strain of $M$. tuberculosis (357). IL-1 $\beta$ released from infected macrophages can also stimulate other cells like lung epithelial cells or uninfected macrophages through IL-1RI to express antimicrobial peptides. These peptides are then secreted, leading to decreased bacterial numbers and increased survival of infected macrophages $(239,358)$. Addition of IL-1 $\beta$ to murine macrophages also increases killing of virulent $M$. tuberculosis by inducing autophagy (359) and leads to increased maturation of mycobacterial phagosomes (167). In line with the in vitro data, there are many studies showing the importance of IL-1 $\beta$, IL-1RI and downstream signaling through MyD88 during tuberculosis in vivo. When disrupting different steps involved in IL-1 $\beta$ signaling, pronounced phenotypes are seen. For example, MyD88deficient mice have severely impaired ability to control bacterial growth, show large necrotic lesions, have defective granuloma formation and succumb to the infection within four weeks despite being able to produce IFN- $\gamma$ (360-361). Though MyD88 also acts as an adaptor for TLR signaling, mice deficient in IL-1RI have a phenotype very similar to MyD88-deficent mice with necrotic lesions, increased bacterial burden 
and death within four weeks, suggesting that much of the protective effects of MyD88 signaling originate from IL-1RI rather than TLRs (362-363). Much of the signal through IL-1RI has been attributed to IL- $1 \beta$, and mice lacking IL-1 $\beta$ succumb to tuberculosis as fast as MyD88-deficient or IL-1RI-deficient mice and display almost as high bacterial burden (363). However, mice deficient in IL-1 $\alpha$ are also highly susceptible to $M$. tuberculosis and the two IL-1 cytokines might have non-redundant roles both relying on signaling through IL-1RI (364). The prerequisite of the NLRP3 inflammasome for IL-1 $\beta$ production in macrophages in vitro, and the crucial role for IL-1 $\beta$ signaling in vivo, strongly suggest a protective and important role for the NLRP3 inflammasome and caspase- 1 activation during tuberculosis. However, several studies have failed to show an effect on bacterial burden, survival or even IL-1 $\beta$ production in mice lacking NLRP3 or caspase-1, and very small effects have been observed in mice lacking the adaptor protein ASC (354-356, 363). Though not fully understood, the reduction of IL-1 $\beta$ production as a consequence of loss of NLRP3, ASC or caspase- 1 can be completely rescued by other pathways in vivo, such as caspase-1-independent production of IL-1 $\beta$ in DCs (307) or extracellular processing by neutrophil proteases (308). Instead, activation of NLRP3 during mycobacterial infection may contribute to inflammation, tissue destruction and severity of the disease. ESX-1-expressing $M$. marinum infecting rat tails form granulomas that share many similarities with human granulomas and in this model, NLRP3-dependent production of IL-1 $\beta$ leads to more pathology without restricting bacterial growth (365). This observation is supported by the fact that IFN- $\gamma$, crucial for protection against $M$. tuberculosis, can limit NLRP3 activation in vivo through NO, which nitrosylates NLRP3, thus suppressing IL-1 $\beta$ production and limiting pathology and tissue damage (298).

Although we are starting to understand the role of the inflammasome and IL- $1 \beta$ in experimentally infected mice and in murine macrophages, the fact remains that $M$. tuberculosis is a human pathogen and the role of the inflammasome in human tuberculosis is still to be elucidated. Mechanisms for inflammasome regulation are more diverse in humans compared to mice, which indicates that the inflammasome plays a different role during human tuberculosis. 


\section{Al MS}

The overall aim of this thesis was to gain insight into the interaction between human macrophages and $M$. tuberculosis and to investigate how the innate immune response is responsible for a host-beneficial outcome of infection. More specifically, the thesis addresses if the NLRP3 inflammasome and IL-1 $\beta$ signaling could shift the balance in favor of the host. The more specific aims were:

- To develop a model system to follow the interaction between human macrophages and virulent $M$. tuberculosis over several days of infection.

- To identify novel ways of enhancing the function of the NLRP3/IL-1 $\beta$ axis in human macrophages infected with M. tuberculosis.

- To evaluate the role of IL-1 $\beta$ production and increased NLRP3 activity in the outcome of infection with respect to bacterial growth, host immune responses and macrophage cell death. 


\section{RESULTS AND \\ DI SCUSSI ON}

\section{Paper I}

One central aspect of the interaction between $M$. tuberculosis and the human macrophage is the ability of the macrophage to kill the bacteria and thus measuring bacterial numbers becomes an important read-out for the host-pathogen interaction. Determination of the number of viable bacteria after infection of human macrophages is of interest not only for understanding host response but is also of importance for drug discovery. The rationale for testing drugs against bacteria in their true environment, the human macrophage, has several advantages. $M$. tuberculosis adapt to the different challenges posed by the macrophage and induce different transcriptional responses controlling metabolism, replication and expression of proteins implicated in virulence of the bacterium depending on the stage of infection (173-174, 366-367). This suggests that the bacteria present inside macrophages have phenotypic differences in the susceptibility to potential drugs compared to bacteria cultured in broth. Testing compounds against intracellular bacteria might also lead to the discovery of novel antimicrobial agents that, instead of being directly bactericidal, act as virulence blockers that prevent intracellular growth or immunomodulators that act through enhancing the antimicrobial capacity of the macrophage itself. To determine the number of viable bacteria after infection, the most commonly used method is plating and culturing defined volumes of lysed cells followed by the counting of colonyforming units (CFU) on agar plates. CFU plating is rather sensitive and allows detection of very low numbers of bacteria. However, to analyze colony-forming units, bacteria need to replicate multiple times before colonies start to appear and this becomes a major drawback when studying very slow-growing bacteria such as $M$. tuberculosis, since it takes 2-3 weeks for a colony of $M$. tuberculosis to appear. Furthermore, CFU plating is rather labor-intensive and it is difficult to handle a large number of samples. With this in mind, we set out to develop a method that could replace CFU plating and detect bacteria directly in cell lysates. It needed to be sensitive enough to detect changes in bacterial numbers and only measure live bacteria as well as be time- and cost-effective. To be able to follow another important aspect of the infection, namely the status of the infected cells, the method needed to include a way of measuring macrophage viability. 
The method is based on the use of a virulent strain of M. tuberculosis, H37Rv, which carries a plasmid, pSMT1, containing the genes for Vibrio harveyi luciferase (H37Rvlux). The plasmid has been previously used for rapid measurement of the number of mycobacteria in mouse spleen, liver and lungs (368-370) and to measure mycobacterial growth in murine macrophages (371). The choice of Vibrio harveyi luciferase was based on two important factors. First, the luminescent reaction involves the oxidation of a long-chain fatty aldehyde and the co-factor riboflavin phosphate $\left(\mathrm{FMNH}_{2}\right)$, which is only present in live bacteria (371). Secondly, the long-chain fatty aldehyde, $n$-decanal, used as a substrate for the reaction is very inexpensive, making it suitable for analyzing a large number of samples. The experiments were performed by seeding macrophages, which had been allowed to differentiate for seven day in $10 \%$ human serum, in 96-well plates. Following incubation overnight, macrophages were infected by allowing the cells to phagocytose H37Rv-lux at a multiplicity of infection (MOI) of 10 for one hour, before medium was changed. At the given time points, a fraction of the supernatant was transferred to white 96-well plates suitable for measuring luminescence, before cells were hypotonically lysed. Flash-luminescence in both supernatant and lysates was measured by a luminometer equipped with an automatic injector, which added the substrate $n$-decanal. Viability of the infected macrophages was measured in a parallel 96-well plate by a calcein acetoxymethyl (AM)-based assay. Calcein AM is taken up by live cells where esterases remove the acetoxymethyl group, leading to a large increase in fluorescence and trapping the molecule intracellularly.

Infection of macrophages with H37Rv-lux at MOI 10 followed by incubation for up to three days resulted in growth of the bacteria in the cell lysates and this growth could be detected by both luminometry and CFU plating. Furthermore, the data obtained by luminometry correlated very well with microscopy data where intracellular GFPexpressing bacteria were measured over the same period of time (data not shown). By calculating the coefficient of variation, the variability between different experiments (interassay) as well as within the same experiment (intra-assay) of both CFU plating and luminometry were evaluated. The variability between experiments was high for both methods, which was expected due to donor-specific differences. However, the intra-assay variability was lower with luminometry compared to CFU plating. The range and detection limit were also compared by measuring a serial dilution of bacterial culture using both luminometry and CFU plating. This showed that CFU plating had the lowest detection limit and can detect down to a single colony, but required additional dilutions to reach concentrations were single colonies are distinguishable. Luminometry was not as sensitive, but had a wide range where a linear relationship between bacterial numbers and arbitrary light units (ALU) was seen. This linear relationship was stable down to approximately 5000 bacteria. Using luminescence, we also measured the supernatant fraction of the infected wells, thereby 
monitoring the total growth in each individual well. To assert that the luminometry method could measure differences in bacterial number, macrophages were either infected with the avirulent strains H37Ra or BCG carrying the pSMT1 plasmid or treated with antibiotics following infection by H37Rv. Calculating the fold-change of bacterial growth by normalizing luminescence values to the initial value at the start of infection showed that both addition of antimycobacterial drugs and infection with avirulent strains lead to decreased growth. Lastly, using calcein AM to measure macrophage viability in our 96-well format showed that infected cells die over the course of infection and at three days post-infection, less than $20 \%$ of the infected macrophages were viable.

When comparing the luminometry and CFU plating, we had to adopt the CFU plating to a 96-well format, meaning that the volumes used for dilutions were rather small. It is likely that the CFU plating would have shown less variability when using larger volumes. Nevertheless, this underlines the problem with scaling up CFU plating to allow analysis of a large number of samples. Furthermore, the luminometry-based method using 96-well plates requires large number of cells and when studying human primary macrophages, the availability of cells becomes a major bottle-neck. For largescale projects, the use of cell lines would be a prerequisite. Since publishing this method, we have further developed it to include long-time infections with a lower MOI as well as decreasing the number of required cells by measuring viability and luminescence serially in the same wells. In conclusion, the luminometry-based method is an efficient, labor- and cost-effective way of measuring growth of $M$. tuberculosis during macrophage infection. This method has been used in subsequent studies in our group, including the other four papers presented in this thesis.

\section{Paper II}

M. tuberculosis infecting a human macrophage avoids the antimicrobial effects of the macrophage and uses the cell as a site for replication. To do so, M. tuberculosis needs to keep the cell alive and has evolved several ways to inhibit apoptosis (223-226). However, at a certain point, the replicating bacteria inside the macrophage need to spread to new cells. To that end, the bacteria are able to induce cell death with necrotic-like features, which is mediated through secretion of ESAT-6 (37-38, 184, 217-218). The fact that ESAT-6 is also required for activation of the NLRP3 inflammasome in macrophages (353) lead us to hypothesize that the cell death in our model (see Paper I) is one of the inflammasome-related pyroptosis or pyronecrosis, which are dependent on caspase-1 or cathepsin B, respectively (311). 
Following infection with $M$. tuberculosis at MOI 10, macrophage viability was determined by staining with calcein AM and bacterial growth was monitored by measuring luminescence. To elucidate if cell death was correlated to bacterial burden, macrophages were also infected at MOI 1. Although the bacteria grew at both MOIs, samples infected at MOI 10 displayed increased growth as compared to MOI 1. In line with this, cells started to lose viability after one day of infection at MOI 10 while remaining intact for up to three days at MOI 1 . One important feature of pyroptosis and pyronecrosis is the release of IL-1 $\beta$. We therefore measured the levels of IL- $1 \beta$ during infection and MOI 10 induced pronounced caspase-1-dependent IL-1 $\beta$ production, while MOI 1 infected cells produced very low amounts of IL- $1 \beta$. Furthermore, to see if production of IL- $1 \beta$ was strictly dependent on MOI, a titration of the MOI was performed. Though a concentration-dependent increase in IL- $1 \beta$ could be observed, infection around MOI 8 led to a significant increase in the levels of IL$1 \beta$, suggesting a threshold for excessive inflammasome activation or cell death. After establishing that the cell death coincided with the release of IL-1 $\beta$, we characterized the features of this cell death with respect to DNA fragmentation, plasma membrane permeability, mitochondrial membrane potential and release of the nuclear protein high-mobility group protein 1 (HMGB1). These experiments showed that H37Rv at MOI 10 induces extensive cell death, with all features present. Though the caspase- 1 and cathepsin B inhibitors were able to block chemically induced cell death or the production of IL-1 $\beta$, they failed to inhibit cell death induced by H37Rv at MOI 10. In contrast, H37Rv at MOI 1 did not induce any significant cell death two days postinfection. Experiments using mutants lacking ESAT-6 showed that this cell death is dependent on ESAT-6 and that even a high load of ESAT-6-deficient bacteria fails to induce cell death. Taken together, we concluded that H37Rv at MOI 10 induces necrosis in an ESAT-6-dependent manner in human macrophages.

Defining the events responsible for the induction of macrophage cell death is an important issue in tuberculosis. Inhibition of apoptosis as an early protective measure and induction of necrosis in later stages, like in the center of a mature granuloma, represents important parts of the pathogenesis of M. tuberculosis. In our study, we observed a sharp increase in cell death upon infection with $M$. tuberculosis that we defined as necrosis, which was accompanied by large amounts of IL-1 $\beta$. In contrast, infection with a lower MOI of 1 did not affect cell death and induced only very low levels of IL-1 $\beta$, suggesting that the intracellular bacterial load is crucial for induction of necrosis and inflammasome activation. Earlier studies have suggested that low numbers of $M$. tuberculosis do not induce cell death, but rather inhibit apoptosis, while higher numbers induce a rather atypical combination of necrotic and apoptotic features, which could be a sign of widespread membrane damage (217-218, 372). After the publication of our paper, a similar study was published also showing that this atypical necrosis is independent of caspase- 1 and cathepsin B, indicating that 
pyroptosis or pyronecrosis are not induced. This study did however find an increase in lysosomal membrane permeability early during cell death induced by virulent bacteria, implicating a lysosomal death pathway (373). Though our results do not point towards the previously described inflammasome-mediated pyronecrosis, which is dependent on cathepsin B, another subsequent study have suggested that the ESAT-6-dependent necrosis is in fact mediated through NLRP3 without involvement of cathepsin B. The authors propose a model where lysosomal membrane damage is a subsequent side effect of the main function of ESAT-6, which is to damage the phagolysomal membrane, leading to activation of NLRP3 and necrotic cell death (374). In line with this, a functional ESX-1 and ESAT-6 is required for translocation of virulent $M$. tuberculosis into the cytosol, where another secretion system, ESX-5, seems to be responsible for the actual NLRP3 activation leading to both caspase-1 activation and caspase-1-independent cell death (42).

In summary, our results support a model where low numbers of ESAT-6-expressing do not elicit any substantial immune responses nor affect the viability of macrophages while at a certain critical threshold of bacterial numbers, atypical necrosis is induced, which is accompanied by excessive inflammasome activation and IL-1 $\beta$ production. Subsequent studies have indicated that when this critical threshold is reached, ESAT-6 through ESX-1 secretion damages phagosomal and lysosomal membranes and translocates into the cytosol where ESX-5 activates NLRP3, leading to both IL-1 $\beta$ production and necrosis. Though we together with other groups were unable to show any involvement of cathepsin B in this type of cell death $(218,373)$, some studies suggest that cathepsin B might play a role (42, 217). Nevertheless, necrosis induced in macrophages by $M$. tuberculosis coincides with inflammasome activation without showing features of either pyroptosis or pyronecrosis. The role of NLRP3 activation in a potential non-canonical pathway in $M$. tuberculosis-induced cell death needs further studies.

\section{Paper III}

The idea that novel antimycobacterial agents acting as immunomodulators or virulence blockers could complement or even replace traditional antibiotics fits well with the fact that the number of $M$. tuberculosis strains that are resistant to one or more of the commonly used antibiotics for treating tuberculosis is increasing. However, the idea of treating with immunomodulating agents that enhance protective host responses is far from new. For example, many different nutritional adjuvants such as minerals, vitamins and amino acids have been investigated for a potential role as immunoenhancers (375-378). One of the most studied of these potential nutritional adjuvants is vitamin $\mathrm{D}$ and an association between susceptibility to tuberculosis and 
vitamin D insufficiency is a long-standing dogma in tuberculosis research (379). Clinical studies with high single doses of vitamin D as a complement to regular antibiotic treatment in tuberculosis patients have so far been inconclusive, ranging from faster sputum conversion in a subgroup with specific VDR polymorphisms (375) to no observed effects at all (378). However, numerous in vitro studies have shown that vitamin $\mathrm{D}$ is able to induce increased killing of mycobacteria in human monocytes and macrophages (49,131, 200,357, 380-384), and have suggested that the major function of vitamin $\mathrm{D}$ is to induce the expression of antimicrobial peptides such as human cathelicidin and beta-defensin 4. However, most of these studies have been performed in human cell lines or monocytes from healthy donors and none has studied whether or not the antimicrobial effects of vitamin D stimulation are altered in macrophages from patients with active tuberculosis.

Thirty-five patients with persistent cough and/or exposure to tuberculosis were included in the study and classified as non-tuberculosis, previous tuberculosis, latent tuberculosis or current. The number of subjects included in each category varied, but no differences with respect to age, gender, ethnicity or serum levels of 25hydroxyvitamin D were seen between the groups. Macrophages were infected with virulent $M$. tuberculosis at MOI 10 before incubated in the presence or absence of 1,25-hydroxyvitamin D for two days. Luminometric analysis of bacterial numbers showed variation in both phagocytosis and bacterial growth between individual subjects and no difference was seen between the different subject groups. However, when comparing the effect of 1,25-hydroxyvitamin D on bacterial growth within the different subject groups, a small but significant decrease in bacterial growth was seen in the group with active tuberculosis. To elucidate whether the increased capacity to control growth of $M$. tuberculosis by macrophages from tuberculosis patients could be coupled to differences in inflammatory responses, the levels of IL-1 $\beta$, TNF, IL-10 and IL-12p40 were measured in culture supernatants. The results showed a tendency of increased IL-1 $\beta$ and IL-10 in all subject groups when cells were stimulated with 1,25hydroxyvitamin D. Increased levels of IL- $1 \beta$ was pronounced in the non-tuberculosis group and IL-10 in the group with active tuberculosis. The ability of 1,25hydroxyvitamin to augment the cytokine response of the infected macrophage was further strengthened by the fact that a combined analysis comparing the differences in cytokine levels in the whole population showed a clear increase in both IL-1 $\beta$, IL-10 and IL-12p40.

Though limited by the small number of patients included, the study shows that active vitamin D increases production of several cytokines from human macrophages, including IL- $1 \beta$. In line with this, vitamin D-mediated production of IL-1 $\beta$ in activated human macrophages has been shown before $(358,385)$. The connection between vitamin D stimulation, IL-1 $\beta$ and bacterial killing has been studied on a mechanistic 
level by Liu et al, who have shown that priming of macrophages, e.g. by TLR1/2 stimulation, induce two different pathways important for antimycobacterial responses. First, this priming step led to upregulation of the VDR and CYP27B1-hydroxylase, the enzyme responsible for converting inactive 25-hydroxyvitamin D to active 1,25hydroxyvitamin D. This upregulation increased the responsiveness of the macrophage towards vitamin D. Secondly, the priming sensitized the cells to IL-1 $\beta$ by upregulating the expression of IL-1R1. IL-1 $\beta$ together with vitamin D was subsequently shown to be required for expression of beta-defensin 4, which in turn increased the killing capacity of the cell (357). Our study showed that cells from patients with active tuberculosis display increased responses to vitamin $\mathrm{D}$, leading to increased growth control of M. tuberculosis. It is tempting to speculate that macrophages from patients with an ongoing infection are primed in vivo, leaving them more responsive to both vitamin D and IL-1 $\beta$ stimulation, which leads to increased control of bacterial growth. In vivo priming or epigenetically imprinted "memory" of innate cells have been observed in studies showing that macrophages cultured for seven days from patients with active tuberculosis have increased mRNA levels of IL-1 $\beta$ and NLRP3 compared to healthy controls (353) and that monocytes from BCG-vaccinated individuals produce more IL-1 $\beta$ and TNF compared to non-vaccinated subjects several months after the vaccination (386). The latter study is well in line with unpublished data from our lab showing that TNF production is increased in macrophages from BCGvaccinated individuals following stimulation with heat-killed $M$. tuberculosis compared to non-vaccinated subjects.

Taken together, our study suggests that macrophages from patients with active tuberculosis are more responsive to vitamin $\mathrm{D}$, displaying enhanced production of cytokines and increased killing of $M$. tuberculosis. We speculate that vitamin D acts together with IL- $1 \beta$ to induce the expression of antimicrobial peptides and these effects are more prominent in cells from patients with active tuberculosis due to an in vivo priming step that upregulates both VDR and IL-1RI expression. For future studies, the expression of antimicrobial peptides in macrophages from tuberculosis patients stimulated with vitamin $\mathrm{D}$ would be of interest.

\section{Paper IV}

During in vivo infection with $M$. tuberculosis, resident alveolar macrophages are the first cells to recognize and respond to the presence of the bacteria. However, other cells are quickly recruited from the circulation and the most abundant of these are neutrophils. Neutrophils are known to phagocytose M. tuberculosis at the site of infection and are an important part of the early response against this pathogen. Uptake of mycobacteria by neutrophils leads to killing of the bacteria as well as induction of 
apoptosis. However, non-infected neutrophils at the site of infection are still shortlived cells and undergo apoptosis spontaneously within 24 hours after leaving circulation (231-232). After induction of apoptosis, the apoptotic neutrophils are recognized and cleared by other adjacent phagocytes. While studies in our lab have shown that uptake of neutrophils, which are apoptotic as a consequence of phagocytosing mycobacteria, by human macrophages induces a proinflammatory response in the macrophage (387-389), uptake of spontaneously apoptotic neutrophils have long been implicated in anti-inflammatory responses and resolution of inflammation (390). However, since the site of infection at early stages likely contains both mycobacteria-infected and spontaneously apoptotic neutrophils, a downregulation of macrophage responses by the latter could have detrimental effects for the host. We therefore investigated the effect of spontaneously apoptotic neutrophils on inflammatory responses in infected macrophages.

Most parts of this study was performed using a model where human macrophages were stimulated with M. tuberculosis, spontaneously apoptotic neutrophils (PMNapo) or both and incubated for 18-20 hours before cells and supernatants were harvested for analysis of cytokines or gene expression. This study and earlier work from the lab concerning the interaction between human macrophages and apoptotic neutrophils during $M$. tuberculosis infection have been performed with $\gamma$-irradiated $M$. tuberculosis, which will be referred to as $\gamma$-Mtb throughout this chapter. Infection of macrophages by $\gamma$-Mtb alone at an MOI of 5 induced expression of most inflammatory genes studied, but addition of PMNapo at a ratio of 2:1 led to an additional increase in the expression of several of those genes including NLRP3, proIL-1 $\beta$, proIL-18, TNF, IL-6, IL-23 $\alpha$ and IL-10. Similar results were obtained when analyzing cytokines on a protein level, where IL-1 $\beta$, TNF, IL-6 and IL-10 displayed significantly higher levels when adding PMNapo to the infected macrophages. This augmenting effect was specifically dependent on phagocytosis of apoptotic cells, since inhibition of actin polymerization led to lower TNF levels after addition of PMNapo. Furthermore, phagocytosis of an inert prey, such as latex beads, did not increase TNF levels in infected macrophages. After establishing that PMNapo enhanced macrophage activation, we continued by investigating if addition of PMNapo could also enhance the killing capacity of macrophages. For these experiments, PMNapo were added to macrophages infected with live luciferase-expressing $M$. tuberculosis and bacterial growth was monitored over several days. The results revealed that PMNapo did indeed induce a significant decrease in mycobacterial growth after a few days of infection, showing that addition of PMNapo had a functional effect. Since several of the genes upregulated after stimulating macrophages with PMNapo were related to the inflammasome and production of IL- $1 \beta$, we decided to more closely examine the role of IL-1 $\beta$ in our model. We first analyzed the production of IL- $1 \beta$ in individual cells which had taken up both $\gamma$-Mtb and PMNapo, and though the double-positive 
population in the samples stimulated with both bacteria and apoptotic cells was small (1\%), we observed a significant increase in the number of IL-1 $\beta$ positive cells compared to cells that had phagocytosed bacteria only. This effect was not observed for TNF production. Lastly, by using YVAD to inhibit caspase-1-mediated IL-1 $\beta$ production, we observed almost a complete loss of IL- $1 \beta$ together with significantly reduced levels of TNF and IL-6 from infected macrophages, showing that IL- $1 \beta$ is to some extent driving the production of other proinflammatory cytokines during infection. More importantly, addition of PMNapo in the presence of YVAD did not influence the levels of TNF and IL-6, suggesting that the augmenting effect acts through increased levels of IL-1 $\beta$.

Our study challenges the earlier view of spontaneously apoptotic neutrophils as inducers of anti-inflammatory responses and we show that during an infection they are instead acting as to enhance cytokine production from infected macrophages and to increase the killing capacity of the macrophage. The phenomenon of increased killing by addition of apoptotic neutrophils has been observed before and has been attributed to the transfer of granular content from the neutrophil to the macrophage (50). We can not rule out that this mechanism does play a major role in the increased killing observed in our model, but we argue that enhancement of macrophage responses by apoptotic neutrophils represents an alternative or complementary mechanism. Uptake of apoptotic cells by macrophages during $M$. tuberculosis infection has been shown to increase phagosomal maturation and increase bacterial killing (230). A recent followup study further showed that recombinant IL- $1 \beta$ increases both the production of TNF and upregulates the surface expression of the TNF receptor-1 (TNFR1) in macrophages. This increase in TNF signaling in turn induces caspase-3 activation and apoptosis of infected macrophages, enabling increased control of mycobacterial growth through efferocytosis (391). These studies support the idea that, in addition to directly bestowing the macrophage with antimicrobial effector molecules, apoptotic neutrophils also act as danger signal, which increases expression of proIL-1 $\beta$ and inflammasome components, and augments the secretion of IL-1 $\beta$ from infected macrophages. This increase in IL-1 $\beta$ can not only lead to direct effects like increased phagosomal maturation and autophagy $(167,359)$, but also by enhancing other proinflammatory responses from the macrophage, such as TNF production, which in turn inhibits bacterial growth.

\section{Paper V}

The role of inflammasome activation in human tuberculosis has for obvious reasons not been studied as thoroughly as in murine tuberculosis, where NLRP3 activation plays a minor role during tuberculosis (354-356, 363). However, polymorphisms in 
inflammasome-related genes such as NLRP3 or in the gene encoding the regulatory protein CARD8 are common in humans and contribute to susceptibility to inflammatory disease caused by excessive IL- $1 \beta$ production. For example, the Q705K polymorphism in NLRP3 leads to spontaneous IL-1 $\beta$ from THP-1 cells (392) and combined with the truncating polymorphism $\mathrm{C} 10 \mathrm{X}$ in $C A R D 8$, it has been linked to increased risk and severity of Crohn's disease and arthritis (302, 304). Furthermore, healthy double-carriers of Q705K and C10X show increased plasma concentrations of IL-1 $\beta$ compared to individuals carrying only one or none of the polymorphisms (393). Despite predisposing for inflammatory disease, the high frequency of polymorphisms such as Q705K and C10X in the Swedish population (6.5\% and 34\%, respectively) suggests a beneficial function of these polymorphisms, traits that have undergone positive selection throughout human evolution. Thus, we hypothesized that polymorphisms in inflammasome-related genes lead to increased protection against infections with intracellular bacteria, such as M. tuberculosis.

Recent studies on the inflammasome led to the identification of patients with autoinflammatory disease who carried polymorphisms in NLRP3 and/or CARD8 and whose monocytes spontaneously produced IL-1 $\beta$. The first patient identified was a young man with recurring fevers, who carried a combination of Q705K and C10X and who improved drastically following administration of anakinra (394). The second set of patients was two siblings also experiencing inflammatory symptoms such as recurring fever. They both carried a novel M299V mutation in NLRP3, where the male sibling, who displayed a more severe clinical picture, also carried the C10X polymorphism in CARD8 (395). To test if gain-of-function alterations in inflammasome genes played a role during $M$. tuberculosis infection, macrophages from these three patients were infected and compared to cells from control individuals. These experiments showed that cells from the two patients harboring genetic variants in NLRP3 combined with the C10X polymorphism in CARD8 controlled growth of $M$. tuberculosis better than control cells and that this correlated with increased phagosomal maturation. The genetic variants in NLRP3 are thought to lead to increased inflammasome activation, while the C10X polymorphism in CARD8 leads to truncation of the protein and it can no longer act as an inhibitor of caspase-1 or NF- $\mathrm{kB}$ activity $(290,301,396)$. We therefore hypothesized that having genetic variants of both NLRP3 and CARD8 led to a scenario where IL-1 $\beta$ was spontaneously produced during differentiation of patient cells into macrophages and that this generated macrophages more capable of handling growth of $M$. tuberculosis. Thus, we speculated that addition of recombinant IL- $1 \beta$ during differentiation of macrophages from healthy blood donors would mimic the milieu in which the patient cells were differentiated. Though we were able to show that IL- $1 \beta$ signaling is an important factor in controlling growth of $M$. tuberculosis during the infection, addition of exogenous recombinant IL-1 $\beta$ during differentiation failed to affect bacterial growth or 
phagosomal maturation. To find an alternative model that could mimic the scenario with the patient cells, we took advantage of the fact that carriers of the combined Q705K and C10X polymorphisms are relatively common in the common Swedish population. To that end, we prepared and infected macrophages from 43 healthy blood donors and measured bacterial growth over two days of infection. After we obtained growth data from all donors, we continued by genotyping them for Q705K and C10X. When comparing bacterial growth between cells from wild type individuals and double-carriers, healthy asymptomatic carriers of both polymorphisms control growth better, while cells from carriers of C10X alone showed increased growth compared to wild type control cells.

To our knowledge, this is the first study showing a beneficial effect of polymorphisms linked to increased inflammasome activity and IL-1 $\beta$ production during human tuberculosis. Furthermore, the direct effect of these polymorphisms at the macrophage level suggests that increased inflammasome activity leads to increased bacterial killing in the very early stages of infection, during the first interactions between the bacteria and the innate immune system. However, if the bacteria manage to avoid early killing and establish infection, the NLRP3 inflammasome becomes redundant, as suggested by in vivo studies in mice, where infection is experimentally established. The most studied effector mechanism of inflammasome activation is the generation of IL- $1 \beta$, and addition of recombinant IL- $1 \beta$ has been shown to induce phagosomal maturation, autophagy and a robust TNF response leading to increased killing of $M$. tuberculosis in macrophages $(167,359,391)$. However, these studies have mainly used murine macrophages and BCG, or a model with low MOI, longer incubation times and no detectable necrosis. The different cells and models used might explain our inability to reproduce the effects of adding recombinant IL-1 $\beta$. Instead it suggests that in our model the beneficial effect of increased inflammasome activation caused by gain-of-

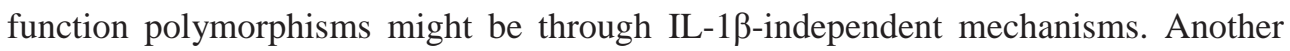
possible explanation is the fact that though a dose-dependent increase of NF- $\mathrm{kB}$ translocation to the nucleus was observed when stimulating with recombinant IL-1 $\beta$, this effect was rather modest compared to TLR stimulation using LPS (data not shown). In addition to the observation that carrying both Q705K and C10X polymorphisms increased bacterial growth control, we also observed less bacterial growth control in cells carrying the C10X polymorphism alone. The mechanism behind this phenomenon needs further investigation, but we speculate that C10X predisposes the cell towards pyroptosis (397), and the necrotic nature of this cell death would permit spreading to adjacent cells and increased replication of M. tuberculosis, while in conjunction with increased NLRP3 activity (e.g. due to the Q705K polymorphism), loss of CARD8 instead increases other inflammasome functions such as cytokine processing, leading to a more favorable outcome for the host. In summary, we propose that polymorphisms that increase the activity of the NLRP3 inflammasome 
and its related functions are a common feature in most human populations due to increased protection against intracellular pathogens such as M. tuberculosis. To confirm the observations in our limited study group and to further investigate the role of gain-of-function polymorphisms in inflammasome components during tuberculosis, it would be of great interest to extend these studies to include tuberculosis patients and their healthy house-hold contacts in endemic settings. 


\section{CONCLUDI NG REMARKS}

Though the disease has been with us throughout history and the causative bacterium has been known for over a century, tuberculosis is still a global health emergency. The biology and lifecycle of the bacterium is linked to a complex host-pathogen interaction, where many factors influence the outcome of the infection. The development of future tools for managing global tuberculosis is dependent on both the discovery of new effective antibiotics and increased knowledge of what constitutes an effective immune response against $M$. tuberculosis. The fact that few exposed individuals develop disease and a substantial part of an exposed population does not develop any adaptive immune responses at all, suggests to us that innate immunity alone is capable of protecting most of us against infection. By finding innate host factors critical for protection against tuberculosis, one can design immunomodulatory agents that stimulate these pathways, acting as adjuvants to traditional antibiotics. It is clear that many innate effector mechanisms exist that contribute to protection against M. tuberculosis. It is also clear that these multifactorial effector mechanisms converge at numerous cross-roads and are intimately connected. This thesis aims at providing increased insight into the interaction between human macrophages and $M$. tuberculosis to identify such protective innate effector mechanisms. More specifically, the thesis focuses on the role of inflammasome activation and IL-1 $\beta$ during infection of human macrophages by $M$. tuberculosis.

The present work has resulted in a hypothetical model, which is summarized in Figure 6. Studying the interaction between the host macrophage and M. tuberculosis was to a large extent made possible by the development of a luminometry-based assay for measuring bacterial growth (Paper I). We show how ESAT-6-expressing bacteria do not induce cell death or elicit any pronounced IL-1 $\beta$ responses from macrophages until a certain threshold of bacteria is reached. At this point, atypical necrosis is induced lacking characteristics of previously described inflammasome-mediated cell death pathways but where the inflammasome and excessive inflammasome IL-1 $\beta$ production occurs simultaneously (Paper II). In addition, we show that vitamin D increases production of IL-1 $\beta$ and increases killing of $M$. tuberculosis specifically in cells from patients with active tuberculosis. We speculate that cells from tuberculosis patients have been primed to express more IL-1RI and VDR, enabling the combination of vitamin D and IL-1 $\beta$ to induce increased expression of antimicrobial peptides such as beta-defensin 4, leading to increased killing of the bacteria (Paper III). By further enhancing the NLRP3/caspase-1/IL-1 $\beta$ axis with apoptotic neutrophils (Paper IV) or through gain-of-function polymorphisms (Paper V), it is possible to increase the killing capacity of the macrophages, potentially through IL-1 $\beta$-independent pathways, 
as well as to enhance production of other proinflammatory mediators, which in turn would have a host-beneficial effect. In conclusion, the work presented in this thesis shows that by enhanced inflammasome activity, it is possible to tip the balance between the human macrophage and M. tuberculosis in favor of the host cell, which might act as a protective mechanism during the early stages of infection.

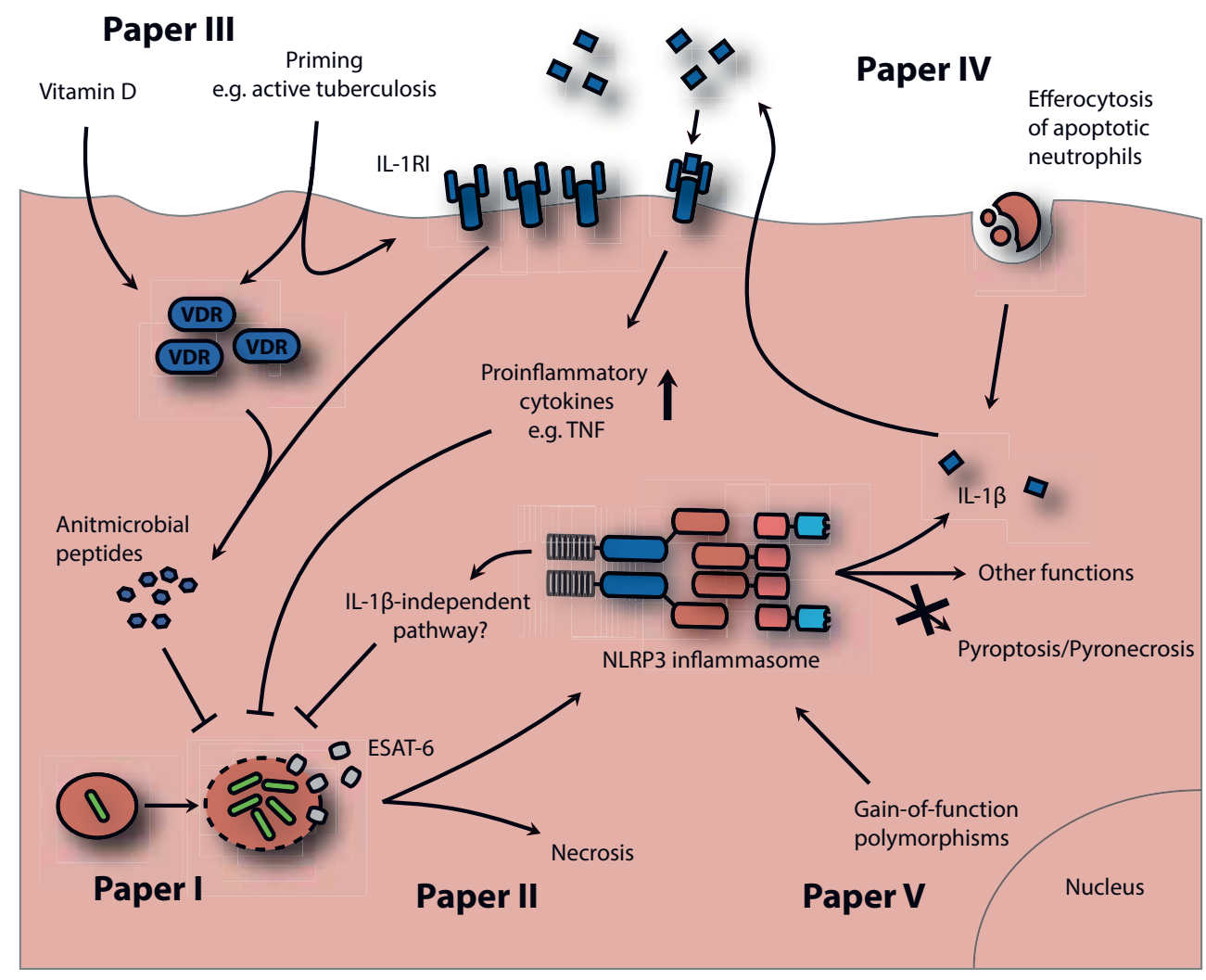

Figure 6. A hypothetical model of the role of the NLRP3 inflammasome and IL-1 $\beta$ during infection by Mycobacterium tuberculosis in human macrophages, based on the findings presented in this thesis. By using a luminometry-based method for measuring bacterial numbers (Paper I) we were able to show that high numbers of ESAT-6-expressing bacteria promote IL-1 $\beta$ production and simultaneously induces necrosis independent of inflammasome activation (Paper II). Activation of the inflammasome and production of IL-1 $\beta$ in conjunction with vitamin $D$ induce increased killing specifically in cells from patients with active tuberculosis (Paper III). Furthermore, increasing inflammasome activity and IL-1 $\beta$ production by uptake of apoptotic neutrophils (Paper IV), or by activating polymorphisms (Paper V), lead to increased production of other proinflammatory cytokines and increased killing capacity of the macrophage. 


\section{REFERENCES}

1. Gutierrez MC, Brisse S, Brosch R, Fabre M, Omais B, Marmiesse M, Supply P, and Vincent V. Ancient origin and gene mosaicism of the progenitor of Mycobacterium tuberculosis. PLoS Pathog. 2005;1(1):e5.

2. Wirth T, Hildebrand F, Allix-Beguec C, Wolbeling F, Kubica T, Kremer K, van Soolingen D, Rusch-Gerdes S, Locht C, Brisse S, et al. Origin, spread and demography of the Mycobacterium tuberculosis complex. PLoS Pathog. 2008;4(9):e1000160.

3. Nerlich AG, Haas CJ, Zink A, Szeimies U, and Hagedorn HG. Molecular evidence for tuberculosis in an ancient Egyptian mummy. Lancet. 1997;350(9088):1404.

4. Salo WL, Aufderheide AC, Buikstra J, and Holcomb TA. Identification of Mycobacterium tuberculosis DNA in a pre-Columbian Peruvian mummy. Proc Natl Acad Sci U S A. 1994;91(6):2091-4.

5. Daniel TM. The history of tuberculosis. Respir Med. 2006;100(11):1862-70.

6. "Physiology or Medicine 1905 - Presentation Speech". Nobelprize.org, http://openx.nobelprize.org/nobel_prizes/medicine/laureates/1905/press.html 17 Apr 2013.

7. "Physiology or Medicine 1952 - Presentation Speech". Nobelprize.org, http://www.nobelprize.org/nobel_prizes/medicine/laureates/1952/press.html 17 Apr 2013.

8. Global tuberculosis report 2012, WHO, http://www.who.int/tb/publications/global_report/gtbr12_main.pdf. 17 Apr 2013.

9. Abubakar I, Zignol M, Falzon D, Raviglione M, Ditiu L, Masham S, Adetifa I, Ford N, Cox H, Lawn SD, et al. Drug-resistant tuberculosis: time for visionary political leadership. Lancet Infect Dis. 2013.

10. Velayati AA, Masjedi MR, Farnia P, Tabarsi P, Ghanavi J, Ziazarifi AH, and Hoffner SE. Emergence of new forms of totally drug-resistant tuberculosis bacilli: super extensively drug-resistant tuberculosis or totally drug-resistant strains in iran. Chest. 2009;136(2):420-5.

11. "Statistik för tuberkulos". Smittskyddsinstitutet.se, http://smi.se/statistik/tuberkulos/. 18 Apr 2013.

12. Lamanna C. The Nature of the Acid-fast Stain. J Bacteriol. 1946;52(1):99-103.

13. Cole ST, Brosch R, Parkhill J, Garnier T, Churcher C, Harris D, Gordon SV, Eiglmeier K, Gas S, Barry CE, 3rd, et al. Deciphering the biology of Mycobacterium tuberculosis from the complete genome sequence. Nature. 1998;393(6685):537-44.

14. Smith NH, Kremer K, Inwald J, Dale J, Driscoll JR, Gordon SV, van Soolingen D, Hewinson RG, and Smith JM. Ecotypes of the Mycobacterium tuberculosis complex. J Theor Biol. 2006;239(2):220-5.

15. Smith NH, Hewinson RG, Kremer K, Brosch R, and Gordon SV. Myths and misconceptions: the origin and evolution of Mycobacterium tuberculosis. Nat Rev Microbiol. 2009;7(7):537-44.

16. Hershberg R, Lipatov M, Small PM, Sheffer H, Niemann S, Homolka S, Roach JC, Kremer K, Petrov DA, Feldman MW, et al. High functional diversity in Mycobacterium tuberculosis driven by genetic drift and human demography. PLoS Biol. 2008;6(12):e311. 
17. Fenner L, Egger M, Bodmer T, Furrer H, Ballif M, Battegay M, Helbling P, Fehr J, Gsponer T, Rieder HL, et al. HIV Infection Disrupts the Sympatric Host-Pathogen Relationship in Human Tuberculosis. PLoS Genet. 2013;9(3):e1003318.

18. Firdessa R, Berg S, Hailu E, Schelling E, Gumi B, Erenso G, Gadisa E, Kiros T, Habtamu M, Hussein J, et al. Mycobacterial lineages causing pulmonary and extrapulmonary tuberculosis, Ethiopia. Emerg Infect Dis. 2013;19(3):460-3.

19. Portevin D, Gagneux S, Comas I, and Young D. Human macrophage responses to clinical isolates from the Mycobacterium tuberculosis complex discriminate between ancient and modern lineages. PLoS Pathog. 2011;7(3):e1001307.

20. Comas I, Chakravartti J, Small PM, Galagan J, Niemann S, Kremer K, Ernst JD, and Gagneux S. Human T cell epitopes of Mycobacterium tuberculosis are evolutionarily hyperconserved. Nat Genet. 2010;42(6):498-503.

21. Brennan PJ. Structure, function, and biogenesis of the cell wall of Mycobacterium tuberculosis. Tuberculosis (Edinb). 2003;83(1-3):91-7.

22. Besra GS, Morehouse CB, Rittner CM, Waechter CJ, and Brennan PJ. Biosynthesis of mycobacterial lipoarabinomannan. J Biol Chem. 1997;272(29):18460-6.

23. Dao DN, Kremer L, Guerardel Y, Molano A, Jacobs WR, Jr., Porcelli SA, and Briken V. Mycobacterium tuberculosis lipomannan induces apoptosis and interleukin-12 production in macrophages. Infect Immun. 2004;72(4):2067-74.

24. Vignal C, Guerardel Y, Kremer L, Masson M, Legrand D, Mazurier J, and Elass E. Lipomannans, but not lipoarabinomannans, purified from Mycobacterium chelonae and Mycobacterium kansasii induce TNF-alpha and IL-8 secretion by a CD14-toll-like receptor 2-dependent mechanism. J Immunol. 2003;171(4):2014-23.

25. Fratti RA, Chua J, Vergne I, and Deretic V. Mycobacterium tuberculosis glycosylated phosphatidylinositol causes phagosome maturation arrest. Proc Natl Acad Sci U S A. 2003;100(9):5437-42.

26. Rojas M, Barrera LF, Puzo G, and Garcia LF. Differential induction of apoptosis by virulent Mycobacterium tuberculosis in resistant and susceptible murine macrophages: role of nitric oxide and mycobacterial products. J Immunol. 1997;159(3):1352-61.

27. Sibley LD, Hunter SW, Brennan PJ, and Krahenbuhl JL. Mycobacterial lipoarabinomannan inhibits gamma interferon-mediated activation of macrophages. Infect Immun. 1988;56(5):1232-6.

28. Wilkinson KA, Newton SM, Stewart GR, Martineau AR, Patel J, Sullivan SM, Herrmann JL, Neyrolles O, Young DB, and Wilkinson RJ. Genetic determination of the effect of post-translational modification on the innate immune response to the 19 kDa lipoprotein of Mycobacterium tuberculosis. BMC Microbiol. 2009;9(93.

29. Abdallah AM, Gey van Pittius NC, Champion PA, Cox J, Luirink J, VandenbrouckeGrauls CM, Appelmelk BJ, and Bitter W. Type VII secretion--mycobacteria show the way. Nat Rev Microbiol. 2007;5(11):883-91.

30. Gey Van Pittius NC, Gamieldien J, Hide W, Brown GD, Siezen RJ, and Beyers AD. The ESAT-6 gene cluster of Mycobacterium tuberculosis and other high G+C Grampositive bacteria. Genome Biol. 2001;2(10):RESEARCH0044.

31. Lightbody KL, Ilghari D, Waters LC, Carey G, Bailey MA, Williamson RA, Renshaw PS, and Carr MD. Molecular features governing the stability and specificity of functional complex formation by Mycobacterium tuberculosis CFP-10/ESAT-6 family proteins. J Biol Chem. 2008;283(25):17681-90.

32. Abdallah AM, Verboom T, Weerdenburg EM, Gey van Pittius NC, Mahasha PW, Jimenez C, Parra M, Cadieux N, Brennan MJ, Appelmelk BJ, et al. PPE and PE_PGRS proteins of Mycobacterium marinum are transported via the type VII secretion system ESX-5. Mol Microbiol. 2009;73(3):329-40. 
33. Sassetti CM, Boyd DH, and Rubin EJ. Genes required for mycobacterial growth defined by high density mutagenesis. Mol Microbiol. 2003;48(1):77-84.

34. Maciag A, Dainese E, Rodriguez GM, Milano A, Provvedi R, Pasca MR, Smith I, Palu G, Riccardi G, and Manganelli R. Global analysis of the Mycobacterium tuberculosis Zur (FurB) regulon. J Bacteriol. 2007;189(3):730-40.

35. McLaughlin B, Chon JS, MacGurn JA, Carlsson F, Cheng TL, Cox JS, and Brown EJ. A mycobacterium ESX-1-secreted virulence factor with unique requirements for export. PLoS Pathog. 2007;3(8):e105.

36. Renshaw PS, Panagiotidou P, Whelan A, Gordon SV, Hewinson RG, Williamson RA, and Carr MD. Conclusive evidence that the major T-cell antigens of the Mycobacterium tuberculosis complex ESAT-6 and CFP-10 form a tight, 1:1 complex and characterization of the structural properties of ESAT-6, CFP-10, and the ESAT6*CFP-10 complex. Implications for pathogenesis and virulence. $\mathrm{J}$ Biol Chem. 2002;277(24):21598-603.

37. Gao LY, Guo S, McLaughlin B, Morisaki H, Engel JN, and Brown EJ. A mycobacterial virulence gene cluster extending RD1 is required for cytolysis, bacterial spreading and ESAT-6 secretion. Mol Microbiol. 2004;53(6):1677-93.

38. Guinn KM, Hickey MJ, Mathur SK, Zakel KL, Grotzke JE, Lewinsohn DM, Smith S, and Sherman DR. Individual RD1-region genes are required for export of ESAT6/CFP-10 and for virulence of Mycobacterium tuberculosis. Mol Microbiol. 2004;51(2):359-70.

39. Frigui W, Bottai D, Majlessi L, Monot M, Josselin E, Brodin P, Garnier T, Gicquel B, Martin C, Leclerc C, et al. Control of M-tuberculosis ESAT-6 secretion and specific T cell recognition by PhoP. PLoS Pathogens. 2008;4(2).

40. Pym AS, Brodin P, Brosch R, Huerre M, and Cole ST. Loss of RD1 contributed to the attenuation of the live tuberculosis vaccines Mycobacterium bovis BCG and Mycobacterium microti. Mol Microbiol. 2002;46(3):709-17.

41. Bottai D, Di Luca M, Majlessi L, Frigui W, Simeone R, Sayes F, Bitter W, Brennan MJ, Leclerc C, Batoni G, et al. Disruption of the ESX-5 system of Mycobacterium tuberculosis causes loss of PPE protein secretion, reduction of cell wall integrity and strong attenuation. Mol Microbiol. 2012;83(6):1195-209.

42. Abdallah AM, Bestebroer J, Savage ND, de Punder K, van Zon M, Wilson L, Korbee CJ, van der Sar AM, Ottenhoff TH, van der Wel NN, et al. Mycobacterial secretion systems ESX-1 and ESX-5 play distinct roles in host cell death and inflammasome activation. J Immunol. 2011;187(9):4744-53.

43. Abdallah AM, Savage ND, van Zon M, Wilson L, Vandenbroucke-Grauls CM, van der Wel NN, Ottenhoff TH, and Bitter W. The ESX-5 secretion system of Mycobacterium marinum modulates the macrophage response. $J$ Immunol. 2008;181(10):7166-75.

44. Weerdenburg EM, Abdallah AM, Mitra S, de Punder K, van der Wel NN, Bird S, Appelmelk BJ, Bitter W, and van der Sar AM. ESX-5-deficient Mycobacterium marinum is hypervirulent in adult zebrafish. Cell Microbiol. 2012;14(5):728-39.

45. Dheda K, Schwander SK, Zhu B, van Zyl-Smit RN, and Zhang Y. The immunology of tuberculosis: from bench to bedside. Respirology. 2010;15(3):433-50.

46. Lin PL, and Flynn JL. Understanding latent tuberculosis: a moving target. J Immunol. 2010;185(1):15-22.

47. Russell DG, Barry CE, 3rd, and Flynn JL. Tuberculosis: what we don't know can, and does, hurt us. Science. 2010;328(5980):852-6. 
48. Gutierrez MG, Master SS, Singh SB, Taylor GA, Colombo MI, and Deretic V. Autophagy is a defense mechanism inhibiting BCG and Mycobacterium tuberculosis survival in infected macrophages. Cell. 2004;119(6):753-66.

49. Liu PT, Stenger S, Li H, Wenzel L, Tan BH, Krutzik SR, Ochoa MT, Schauber J, Wu $\mathrm{K}$, Meinken $\mathrm{C}$, et al. Toll-like receptor triggering of a vitamin D-mediated human antimicrobial response. Science. 2006;311(5768):1770-3.

50. Tan BH, Meinken C, Bastian M, Bruns H, Legaspi A, Ochoa MT, Krutzik SR, Bloom BR, Ganz T, Modlin RL, et al. Macrophages acquire neutrophil granules for antimicrobial activity against intracellular pathogens. J Immunol. 2006;177(3):186471.

51. Flynn JL, and Chan J. What's good for the host is good for the bug. Trends Microbiol. 2005;13(3):98-102.

52. Ramakrishnan L. Revisiting the role of the granuloma in tuberculosis. Nature Reviews Immunology. 2012;12(5):352-66.

53. Lawn SD, and Zumla AI. Tuberculosis. Lancet. 2011;378(9785):57-72.

54. Lawn SD, Mwaba P, Bates M, Piatek A, Alexander H, Marais BJ, Cuevas LE, McHugh TD, Zijenah L, Kapata N, et al. Advances in tuberculosis diagnostics: the Xpert MTB/RIF assay and future prospects for a point-of-care test. Lancet Infect Dis. 2013;13(4):349-61.

55. Weyer K, Mirzayev F, Migliori G, Van Gemert W, D'Ambrosio L, Zignol M, Floyd K, Centis R, Cirillo D, Tortoli E, et al. Rapid molecular TB diagnosis: evidence, policy-making and global implementation of Xpert(R)MTB/RIF. Eur Respir J. 2012.

56. Moore DA, Evans CA, Gilman RH, Caviedes L, Coronel J, Vivar A, Sanchez E, Pinedo Y, Saravia JC, Salazar C, et al. Microscopic-observation drug-susceptibility assay for the diagnosis of TB. $N$ Engl J Med. 2006;355(15):1539-50.

57. Idh J, Abate E, Westman A, Elias D, Janols H, Gelaw A, Getachew A, Alemu S, Aseffa A, Britton S, et al. Kinetics of the QuantiFERON-TB Gold In-Tube test during treatment of patients with sputum smear-positive tuberculosis in relation to initial TST result and severity of disease. Scand J Infect Dis. 2010;42(9):650-7.

58. Hopewell PC, Pai M, Maher D, Uplekar M, and Raviglione MC. International standards for tuberculosis care. Lancet Infect Dis. 2006;6(11):710-25.

59. Sterling TR, Villarino ME, Borisov AS, Shang N, Gordin F, Bliven-Sizemore E, Hackman J, Hamilton CD, Menzies D, Kerrigan A, et al. Three months of rifapentine and isoniazid for latent tuberculosis infection. N Engl J Med. 2011;365(23):2155-66.

60. Ottenhoff TH, and Kaufmann SH. Vaccines against tuberculosis: where are we and where do we need to go? PLoS Pathog. 2012;8(5):e1002607.

61. Schlesinger LS, Hull SR, and Kaufman TM. Binding of the terminal mannosyl units of lipoarabinomannan from a virulent strain of Mycobacterium tuberculosis to human macrophages. J Immunol. 1994;152(8):4070-9.

62. Schlesinger LS. Macrophage phagocytosis of virulent but not attenuated strains of Mycobacterium tuberculosis is mediated by mannose receptors in addition to complement receptors. J Immunol. 1993;150(7):2920-30.

63. Schlesinger LS, Kaufman TM, Iyer S, Hull SR, and Marchiando LK. Differences in mannose receptor-mediated uptake of lipoarabinomannan from virulent and attenuated strains of Mycobacterium tuberculosis by human macrophages. $J$ Immunol. 1996;157(10):4568-75.

64. Torrelles JB, Azad AK, and Schlesinger LS. Fine discrimination in the recognition of individual species of phosphatidyl-myo-inositol mannosides from Mycobacterium tuberculosis by C-type lectin pattern recognition receptors. $J$ Immunol. 2006;177(3):1805-16. 
65. Martinez-Pomares L. The mannose receptor. J Leukoc Biol. 2012;92(6):1177-86.

66. Nigou J, Zelle-Rieser C, Gilleron M, Thurnher M, and Puzo G. Mannosylated lipoarabinomannans inhibit IL-12 production by human dendritic cells: evidence for a negative signal delivered through the mannose receptor. $J$ Immunol. 2001;166(12):7477-85.

67. Rajaram MV, Brooks MN, Morris JD, Torrelles JB, Azad AK, and Schlesinger LS. Mycobacterium tuberculosis activates human macrophage peroxisome proliferatoractivated receptor gamma linking mannose receptor recognition to regulation of immune responses. J Immunol. 2010;185(2):929-42.

68. Zimmerli S, Edwards S, and Ernst JD. Selective receptor blockade during phagocytosis does not alter the survival and growth of Mycobacterium tuberculosis in human macrophages. Am J Respir Cell Mol Biol. 1996;15(6):760-70.

69. Court N, Vasseur V, Vacher R, Fremond C, Shebzukhov Y, Yeremeev VV, Maillet I, Nedospasov SA, Gordon S, Fallon PG, et al. Partial redundancy of the pattern recognition receptors, scavenger receptors, and C-type lectins for the long-term control of Mycobacterium tuberculosis infection. J Immunol. 2010;184(12):7057-70.

70. Kang PB, Azad AK, Torrelles JB, Kaufman TM, Beharka A, Tibesar E, DesJardin LE, and Schlesinger LS. The human macrophage mannose receptor directs Mycobacterium tuberculosis lipoarabinomannan-mediated phagosome biogenesis. $J$ Exp Med. 2005;202(7):987-99.

71. Schlesinger LS, Bellinger-Kawahara CG, Payne NR, and Horwitz MA. Phagocytosis of Mycobacterium tuberculosis is mediated by human monocyte complement receptors and complement component C3. J Immunol. 1990;144(7):2771-80.

72. Le Cabec V, Carreno S, Moisand A, Bordier C, and Maridonneau-Parini I. Complement receptor 3 (CD11b/CD18) mediates type I and type II phagocytosis during nonopsonic and opsonic phagocytosis, respectively. $J$ Immunol. 2002;169(4):2003-9.

73. Malik ZA, Denning GM, and Kusner DJ. Inhibition of $\mathrm{Ca}(2+)$ signaling by Mycobacterium tuberculosis is associated with reduced phagosome-lysosome fusion and increased survival within human macrophages. J Exp Med. 2000;191(2):287-302.

74. Melo MD, Catchpole IR, Haggar G, and Stokes RW. Utilization of CD11b knockout mice to characterize the role of complement receptor 3 (CR3, CD11b/CD18) in the growth of Mycobacterium tuberculosis in macrophages. Cell Immunol. 2000;205(1):13-23.

75. Soilleux EJ, Morris LS, Leslie G, Chehimi J, Luo Q, Levroney E, Trowsdale J, Montaner LJ, Doms RW, Weissman D, et al. Constitutive and induced expression of DC-SIGN on dendritic cell and macrophage subpopulations in situ and in vitro. $J$ Leukoc Biol. 2002;71(3):445-57.

76. Herrmann JL, and Lagrange PH. Dendritic cells and Mycobacterium tuberculosis: which is the Trojan horse? Pathol Biol (Paris). 2005;53(1):35-40.

77. Brown GD. Dectin-1: a signalling non-TLR pattern-recognition receptor. Nat Rev Immunol. 2006;6(1):33-43.

78. Yadav M, and Schorey JS. The beta-glucan receptor dectin-1 functions together with TLR2 to mediate macrophage activation by mycobacteria. Blood. 2006;108(9):316875.

79. Betz BE, Azad AK, Morris JD, Rajaram MV, and Schlesinger LS. beta-Glucans inhibit intracellular growth of Mycobacterium bovis BCG but not virulent Mycobacterium tuberculosis in human macrophages. Microb Pathog. 2011;51(4):23342. 
80. Marakalala MJ, Guler R, Matika L, Murray G, Jacobs M, Brombacher F, Rothfuchs AG, Sher A, and Brown GD. The Syk/CARD9-coupled receptor Dectin-1 is not required for host resistance to Mycobacterium tuberculosis in mice. Microbes Infect. 2011;13(2):198-201.

81. Botos I, Segal DM, and Davies DR. The structural biology of Toll-like receptors. Structure. 2011;19(4):447-59.

82. Takeda K, and Akira S. Roles of Toll-like receptors in innate immune responses. Genes Cells. 2001;6(9):733-42.

83. Kawai T, and Akira S. TLR signaling. Cell Death Differ. 2006;13(5):816-25.

84. Pahl HL. Activators and target genes of Rel/NF-kappaB transcription factors. Oncogene. 1999;18(49):6853-66.

85. Prados-Rosales R, Baena A, Martinez LR, Luque-Garcia J, Kalscheuer R, Veeraraghavan U, Camara C, Nosanchuk JD, Besra GS, Chen B, et al. Mycobacteria release active membrane vesicles that modulate immune responses in a TLR2dependent manner in mice. J Clin Invest. 2011;121(4):1471-83.

86. Bulut Y, Michelsen KS, Hayrapetian L, Naiki Y, Spallek R, Singh M, and Arditi M. Mycobacterium tuberculosis heat shock proteins use diverse Toll-like receptor pathways to activate pro-inflammatory signals. J Biol Chem. 2005;280(22):20961-7.

87. Kiemer AK, Senaratne RH, Hoppstadter J, Diesel B, Riley LW, Tabeta K, Bauer S, Beutler B, and Zuraw BL. Attenuated activation of macrophage TLR9 by DNA from virulent mycobacteria. J Innate Immun. 2009;1(1):29-45.

88. Heldwein KA, Liang MD, Andresen TK, Thomas KE, Marty AM, Cuesta N, Vogel SN, and Fenton MJ. TLR2 and TLR4 serve distinct roles in the host immune response against Mycobacterium bovis BCG. J Leukoc Biol. 2003;74(2):277-86.

89. Ito T, Schaller M, Hogaboam CM, Standiford TJ, Chensue SW, and Kunkel SL. TLR9 activation is a key event for the maintenance of a mycobacterial antigen-elicited pulmonary granulomatous response. Eur J Immunol. 2007;37(10):2847-55.

90. Reiling N, Holscher C, Fehrenbach A, Kroger S, Kirschning CJ, Goyert S, and Ehlers S. Cutting edge: Toll-like receptor (TLR)2- and TLR4-mediated pathogen recognition in resistance to airborne infection with Mycobacterium tuberculosis. J Immunol. 2002;169(7):3480-4.

91. Holscher C, Reiling N, Schaible UE, Holscher A, Bathmann C, Korbel D, Lenz I, Sonntag T, Kroger S, Akira S, et al. Containment of aerogenic Mycobacterium tuberculosis infection in mice does not require MyD88 adaptor function for TLR2, -4 and -9. Eur J Immunol. 2008;38(3):680-94.

92. Reiling N, Ehlers S, and Holscher C. MyDths and un-TOLLed truths: sensor, instructive and effector immunity to tuberculosis. Immunol Lett. 2008;116(1):15-23.

93. Thoma-Uszynski S, Stenger S, Takeuchi O, Ochoa MT, Engele M, Sieling PA, Barnes PF, Rollinghoff M, Bolcskei PL, Wagner M, et al. Induction of direct antimicrobial activity through mammalian toll-like receptors. Science. 2001;291(5508):1544-7.

94. Tian T, Jin S, Dong J, and Li G. Lack of association between Toll-like receptor 4 gene Asp299Gly and Thr399Ile polymorphisms and tuberculosis susceptibility: a metaanalysis. Infect Genet Evol. 2013;14(156-60.

95. Zhang Y, Jiang T, Yang X, Xue Y, Wang C, Liu J, Zhang X, Chen Z, Zhao M, and Li JC. Toll-like receptor $-1,-2$, and -6 polymorphisms and pulmonary tuberculosis susceptibility: a systematic review and meta-analysis. PLoS ONE. 2013;8(5):e63357.

96. Ting JP, Lovering RC, Alnemri ES, Bertin J, Boss JM, Davis BK, Flavell RA, Girardin SE, Godzik A, Harton JA, et al. The NLR gene family: a standard nomenclature. Immunity. 2008;28(3):285-7. 
97. Hu Z, Yan C, Liu P, Huang Z, Ma R, Zhang C, Wang R, Zhang Y, Martinon F, Miao D, et al. Crystal structure of NLRC4 reveals its autoinhibition mechanism. Science. 2013;341(6142):172-5.

98. Tanabe T, Chamaillard M, Ogura Y, Zhu L, Qiu S, Masumoto J, Ghosh P, Moran A, Predergast MM, Tromp G, et al. Regulatory regions and critical residues of NOD2 involved in muramyl dipeptide recognition. EMBO J. 2004;23(7):1587-97.

99. Mayor A, Martinon F, De Smedt T, Petrilli V, and Tschopp J. A crucial function of SGT1 and HSP90 in inflammasome activity links mammalian and plant innate immune responses. Cytokine. 2007;39(1):26-.

100. Duncan JA. Bergstralh DT. Wang Y. Willingham SB. Ye Z. Zimmermann AG. Ting JP. Cryopyrin/NALP3 Binds ATP/dATP, is an ATPase, and Requires ATP Binding to Mediate Inflammatory Signaling. Proceedings of the National Academy of Sciences of the United States of America. 2007;104(19):8041-6.

101. Tschopp J, and Schroder K. NLRP3 inflammasome activation: The convergence of multiple signalling pathways on ROS production? Nat Rev Immunol. 2010;10(3):2105.

102. Girardin SE, Travassos LH, Herve M, Blanot D, Boneca IG, Philpott DJ, Sansonetti PJ, and Mengin-Lecreulx D. Peptidoglycan molecular requirements allowing detection by Nod1 and Nod2. J Biol Chem. 2003;278(43):41702-8.

103. Park JH, Kim YG, McDonald C, Kanneganti TD, Hasegawa M, Body-Malapel M, Inohara N, and Nunez G. RICK/RIP2 mediates innate immune responses induced through Nod1 and Nod2 but not TLRs. Journal of Immunology. 2007;178(4):2380-6.

104. Cooney R, Baker J, Brain O, Danis B, Pichulik T, Allan P, Ferguson DJ, Campbell BJ, Jewell D, and Simmons A. NOD2 stimulation induces autophagy in dendritic cells influencing bacterial handling and antigen presentation. Nat Med. 2010;16(1):90-7.

105. Travassos LH, Carneiro LA, Ramjeet M, Hussey S, Kim YG, Magalhaes JG, Yuan L, Soares F, Chea E, Le Bourhis L, et al. Nod1 and Nod2 direct autophagy by recruiting ATG16L1 to the plasma membrane at the site of bacterial entry. Nat Immunol. 2010;11(1):55-62.

106. Pandey AK, Yang YB, Jiang ZZ, Fortune SM, Coulombe F, Behr MA, Fitzgerald KA, Sassetti CM, and Kelliher MA. NOD2, RIP2 and IRF5 Play a Critical Role in the Type I Interferon Response to Mycobacterium tuberculosis. PLoS Pathogens. 2009;5(7).

107. Brooks MN, Rajaram MVS, Azad AK, Amer AO, Valdivia-Arenas MA, Park JH, Nunez G, and Schlesinger LS. NOD2 controls the nature of the inflammatory response and subsequent fate of Mycobacterium tuberculosis and M-bovis BCG in human macrophages. Cellular Microbiology. 2011;13(3):402-18.

108. Divangahi M, Mostowy S, Coulombe F, Kozak R, Guillot L, Veyrier F, Kobayashi KS, Flavell RA, Gros P, and Behr MA. NOD2-Deficient Mice Have Impaired Resistance to Mycobacterium tuberculosis Infection through Defective Innate and Adaptive Immunity. Journal of Immunology. 2008;181(10):7157-65.

109. Gandotra S, Jang S, Murray PJ, Salgame P, and Ehrt S. Nucleotide-binding oligomerization domain protein 2-deficient mice control infection with Mycobactefium tuberculosis. Infection and Immunity. 2007;75(11):5127-34.

110. Zhang P, Dixon M, Zucchelli M, Hambiliki F, Levkov L, Hovatta O, and Kere J. Expression analysis of the NLRP gene family suggests a role in human preimplantation development. PLoS ONE. 2008;3(7):e2755.

111. Martinon F, Hofmann K, and Tschopp J. The pyrin domain: a possible member of the death domain-fold family implicated in apoptosis and inflammation. Current Biology. 2001;11(4):R118-R20. 
112. Martinon F, Burns K, and Tschopp J. The inflammasome: a molecular platform triggering activation of inflammatory caspases and processing of proIL-beta. Mol Cell. 2002;10(2):417-26.

113. Boyden ED, and Dietrich WF. Nalp1b controls mouse macrophage susceptibility to anthrax lethal toxin. Nature Genetics. 2006;38(2):240-4.

114. Agostini L, Martinon F, Burns K, McDermott MF, Hawkins PN, and Tschopp J. NALP3 forms an IL-1beta-processing inflammasome with increased activity in Muckle-Wells autoinflammatory disorder. Immunity. 2004;20(3):319-25.

115. Kummer JA, Broekhuizen R, Everett H, Agostini L, Kuijk L, Martinon F, van Bruggen R, and Tschopp J. Inflammasome components NALP 1 and 3 show distinct but separate expression profiles in human tissues suggesting a site-specific role in the inflammatory response. Journal of Histochemistry \& Cytochemistry. 2007;55(5):44352.

116. Schroder K, and Tschopp J. The Inflammasomes. Cell. 2010;140(6):821-32.

117. Zambetti LP, Laudisi F, Licandro G, Ricciardi-Castagnoli P, and Mortellaro A. The rhapsody of NLRPs: master players of inflammation ... and a lot more. Immunologic Research. 2012;53(1-3):78-90.

118. Hugot JP, Chamaillard M, Zouali H, Lesage S, Cezard JP, Belaiche J, Almer S, Tysk C, O'Morain CA, Gassull M, et al. Association of NOD2 leucine-rich repeat variants with susceptibility to Crohn's disease. Nature. 2001;411(6837):599-603.

119. Azad AK, Sadee W, and Schlesinger LS. Innate Immune Gene Polymorphisms in Tuberculosis. Infection and Immunity. 2012;80(10):3343-59.

120. Locksley RM, Killeen N, and Lenardo MJ. The TNF and TNF receptor superfamilies: Integrating mammalian biology. Cell. 2001;104(4):487-501.

121. Bekker LG, Freeman S, Murray PJ, Ryffel B, and Kaplan G. TNF-alpha controls intracellular mycobacterial growth by both inducible nitric oxide synthase-dependent and inducible nitric oxide synthase-independent pathways. $J$ Immunol. 2001;166(11):6728-34.

122. Miller JL, Velmurugan K, Cowan MJ, and Briken V. The type I NADH dehydrogenase of Mycobacterium tuberculosis counters phagosomal NOX2 activity to inhibit TNF-alpha-mediated host cell apoptosis. PLoS Pathog. 2010;6(4):e1000864.

123. Rajaram MVS, Ni B, Morris JD, Brooks MN, Carlson TK, Bakthavachalu B, Schoenberg DR, Torrelles JB, and Schlesinger LS. Mycobacterium tuberculosis lipomannan blocks TNF biosynthesis by regulating macrophage MAPK-activated protein kinase 2 (MK2) and microRNA miR-125b. Proceedings of the National Academy of Sciences of the United States of America. 2011;108(42):17408-13.

124. Flynn JL, Goldstein MM, Chan J, Triebold KJ, Pfeffer K, Lowenstein CJ, Schreiber R, Mak TW, and Bloom BR. Tumor-Necrosis-Factor-Alpha Is Required in the Protective Immune-Response against Mycobacterium-Tuberculosis in Mice. Immunity. 1995;2(6):561-72.

125. Xie X, Li F, Chen JW, and Wang J. Risk of tuberculosis infection in anti-TNF-alpha biological therapy: From bench to bedside. J Microbiol Immunol Infect. 2013.

126. Bekker LG, Moreira AL, Bergtold A, Freeman S, Ryffel B, and Kaplan G. Immunopathologic effects of tumor necrosis factor alpha in murine mycobacterial infection are dose dependent. Infection and Immunity. 2000;68(12):6954-61.

127. Flynn JL, and Chan J. Immunology of tuberculosis. Annu Rev Immunol. 2001;19(93129.

128. Cooper AM, Dalton DK, Stewart TA, Griffin JP, Russell DG, and Orme IM. Disseminated tuberculosis in interferon gamma gene-disrupted mice. $J$ Exp Med. 1993;178(6):2243-7. 
129. Fortune SM, Solache A, Jaeger A, Hill PJ, Belisle JT, Bloom BR, Rubin EJ, and Ernst JD. Mycobacterium tuberculosis inhibits macrophage responses to IFN-gamma through myeloid differentiation factor 88-dependent and -independent mechanisms. $J$ Immunol. 2004;172(10):6272-80.

130. Newport MJ, Huxley CM, Huston S, Hawrylowicz CM, Oostra BA, Williamson R, and Levin M. A mutation in the interferon-gamma-receptor gene and susceptibility to mycobacterial infection. N Engl J Med. 1996;335(26):1941-9.

131. Fabri M, Stenger S, Shin DM, Yuk JM, Liu PT, Lee HM, Krutzik SR, Jo EK, Bloom $\mathrm{BR}$, and Modlin RL. Vitamin D is required for T cell mediated antimicrobial activity of human macrophages. Experimental Dermatology. 2011;20(2):176-7.

132. Goldsack L, and Kirman JR. Half-truths and selective memory: Interferon gamma, CD4(+) $\mathrm{T}$ cells and protective memory against tuberculosis. Tuberculosis. 2007;87(6):465-73.

133. Cooper AM, Solache A, and Khader SA. Interleukin-12 and tuberculosis: an old story revisited. Curr Opin Immunol. 2007;19(4):441-7.

134. Redford PS, Murray PJ, and O'Garra A. The role of IL-10 in immune regulation during M. tuberculosis infection. Mucosal Immunol. 2011;4(3):261-70.

135. Higgins DM, Sanchez-Campillo J, Rosas-Taraco AG, Lee EJ, Orme IM, and Gonzalez-Juarrero M. Lack of IL-10 alters inflammatory and immune responses during pulmonary Mycobacterium tuberculosis infection. Tuberculosis (Edinb). 2009;89(2):149-57.

136. Saunders BM, Frank AA, Orme IM, and Cooper AM. Interleukin-6 induces early gamma interferon production in the infected lung but is not required for generation of specific immunity to Mycobacterium tuberculosis infection. Infect Immun. 2000;68(6):3322-6.

137. Nagabhushanam V, Solache A, Ting LM, Escaron CJ, Zhang JY, and Ernst JD. Innate inhibition of adaptive immunity: Mycobacterium tuberculosis-induced IL-6 inhibits macrophage responses to IFN-gamma. J Immunol. 2003;171(9):4750-7.

138. Sica A, and Mantovani A. Macrophage plasticity and polarization: in vivo veritas. $J$ Clin Invest. 2012;122(3):787-95.

139. Mantovani A, Sica A, Sozzani S, Allavena P, Vecchi A, and Locati M. The chemokine system in diverse forms of macrophage activation and polarization. Trends Immunol. 2004;25(12):677-86.

140. Mosser DM, and Edwards JP. Exploring the full spectrum of macrophage activation. Nat Rev Immunol. 2008;8(12):958-69.

141. Kahnert A, Seiler P, Stein M, Bandermann S, Hahnke K, Mollenkopf H, and Kaufmann SH. Alternative activation deprives macrophages of a coordinated defense program to Mycobacterium tuberculosis. Eur J Immunol. 2006;36(3):631-47.

142. Schreiber T, Ehlers S, Heitmann L, Rausch A, Mages J, Murray PJ, Lang R, and Holscher C. Autocrine IL-10 induces hallmarks of alternative activation in macrophages and suppresses antituberculosis effector mechanisms without compromising T cell immunity. J Immunol. 2009;183(2):1301-12.

143. Raju B, Hoshino Y, Belitskaya-Levy I, Dawson R, Ress S, Gold JA, Condos R, Pine R, Brown S, Nolan A, et al. Gene expression profiles of bronchoalveolar cells in pulmonary TB. Tuberculosis (Edinb). 2008;88(1):39-51.

144. Swanson JA. Shaping cups into phagosomes and macropinosomes. Nat Rev Mol Cell Biol. 2008;9(8):639-49.

145. Vieira OV, Botelho RJ, and Grinstein S. Phagosome maturation: aging gracefully. Biochem J. 2002;366(Pt 3):689-704. 
146. Zerial M, and McBride H. Rab proteins as membrane organizers. Nat Rev Mol Cell Bio. 2001;2(2):107-17.

147. Simonsen A, Gaullier JM, D'Arrigo A, and Stenmark H. The Rab5 effector EEA1 interacts directly with syntaxin-6. J Biol Chem. 1999;274(41):28857-60.

148. Rink J, Ghigo E, Kalaidzidis Y, and Zerial M. Rab conversion as a mechanism of progression from early to late endosomes. Cell. 2005;122(5):735-49.

149. Del Conte-Zerial P, Brusch L, Rink JC, Collinet C, Kalaidzidis Y, Zerial M, and Deutsch A. Membrane identity and GTPase cascades regulated by toggle and cut-out switches. Mol Syst Biol. 2008;4(206.

150. Bright NA, Gratian MJ, and Luzio JP. Endocytic delivery to lysosomes mediated by concurrent fusion and kissing events in living cells. Curr Biol. 2005;15(4):360-5.

151. Kinchen JM, and Ravichandran KS. Phagosome maturation: going through the acid test. Nat Rev Mol Cell Biol. 2008;9(10):781-95.

152. Flannagan RS, Cosio G, and Grinstein S. Antimicrobial mechanisms of phagocytes and bacterial evasion strategies. Nat Rev Microbiol. 2009;7(5):355-66.

153. Fang FC. Antimicrobial reactive oxygen and nitrogen species: concepts and controversies. Nat Rev Microbiol. 2004;2(10):820-32.

154. Vanlandingham PA, and Ceresa BP. Rab7 regulates late endocytic trafficking downstream of multivesicular body biogenesis and cargo sequestration. $J$ Biol Chem. 2009;284(18):12110-24.

155. Bustamante J, Aksu G, Vogt G, de Beaucoudrey L, Genel F, Chapgier A, FilipeSantos O, Feinberg J, Emile JF, Kutukculer N, et al. BCG-osis and tuberculosis in a child with chronic granulomatous disease. J Allergy Clin Immunol. 2007;120(1):32-8.

156. Ma X, Dou S, Wright JA, Reich RA, Teeter LD, El Sahly HM, Awe RJ, Musser JM, and Graviss EA. 5' dinucleotide repeat polymorphism of NRAMP1 and susceptibility to tuberculosis among Caucasian patients in Houston, Texas. Int J Tuberc Lung Dis. 2002;6(9):818-23.

157. Song H, Huff J, Janik K, Walter K, Keller C, Ehlers S, Bossmann SH, and Niederweis M. Expression of the ompATb operon accelerates ammonia secretion and adaptation of Mycobacterium tuberculosis to acidic environments. Mol Microbiol. 2011;80(4):900-18.

158. Vandal OH, Pierini LM, Schnappinger D, Nathan CF, and Ehrt S. A membrane protein preserves intrabacterial $\mathrm{pH}$ in intraphagosomal Mycobacterium tuberculosis. Nat Med. 2008;14(8):849-54.

159. Wong D, Bach H, Sun J, Hmama Z, and Av-Gay Y. Mycobacterium tuberculosis protein tyrosine phosphatase (PtpA) excludes host vacuolar-H+-ATPase to inhibit phagosome acidification. Proc Natl Acad Sci U S A. 2011;108(48):19371-6.

160. Welin A, Raffetseder J, Eklund D, Stendahl O, and Lerm M. Importance of phagosomal functionality for growth restriction of Mycobacterium tuberculosis in primary human macrophages. J Innate Immun. 2011;3(5):508-18.

161. Welin A, Winberg ME, Abdalla H, Sarndahl E, Rasmusson B, Stendahl O, and Lerm M. Incorporation of Mycobacterium tuberculosis lipoarabinomannan into macrophage membrane rafts is a prerequisite for the phagosomal maturation block. Infect Immun. 2008;76(7):2882-7.

162. Fratti RA, Backer JM, Gruenberg J, Corvera S, and Deretic V. Role of phosphatidylinositol 3-kinase and Rab5 effectors in phagosomal biogenesis and mycobacterial phagosome maturation arrest. J Cell Biol. 2001;154(3):631-44.

163. Vergne I, Chua J, Lee HH, Lucas M, Belisle J, and Deretic V. Mechanism of phagolysosome biogenesis block by viable Mycobacterium tuberculosis. Proc Natl Acad Sci U S A. 2005;102(11):4033-8. 
164. Bhat SA, Singh N, Trivedi A, Kansal P, Gupta P, and Kumar A. The mechanism of redox sensing in Mycobacterium tuberculosis. Free Radic Biol Med. 2012;53(8):162541.

165. Siegrist MS, Unnikrishnan M, McConnell MJ, Borowsky M, Cheng TY, Siddiqi N, Fortune SM, Moody DB, and Rubin EJ. Mycobacterial Esx-3 is required for mycobactin-mediated iron acquisition. Proc Natl Acad Sci $U$ S A. 2009;106(44):18792-7.

166. Harris J, Hope JC, and Keane J. Tumor necrosis factor blockers influence macrophage responses to Mycobacterium tuberculosis. J Infect Dis. 2008;198(12):1842-50.

167. Master SS, Rampini SK, Davis AS, Keller C, Ehlers S, Springer B, Timmins GS, Sander P, and Deretic V. Mycobacterium tuberculosis prevents inflammasome activation. Cell Host Microbe. 2008;3(4):224-32.

168. Schaible UE, Sturgill-Koszycki S, Schlesinger PH, and Russell DG. Cytokine activation leads to acidification and increases maturation of Mycobacterium aviumcontaining phagosomes in murine macrophages. J Immunol. 1998;160(3):1290-6.

169. Bruns H, Stegelmann F, Fabri M, Dohner K, van Zandbergen G, Wagner M, Skinner M, Modlin RL, and Stenger S. Abelson tyrosine kinase controls phagosomal acidification required for killing of Mycobacterium tuberculosis in human macrophages. J Immunol. 2012;189(8):4069-78.

170. Idh J, Westman A, Elias D, Moges F, Getachew A, Gelaw A, Sundqvist T, Forslund $\mathrm{T}$, Alemu A, Ayele B, et al. Nitric oxide production in the exhaled air of patients with pulmonary tuberculosis in relation to HIV co-infection. BMC Infect Dis. 2008;8(146.

171. Schon T, Elmberger G, Negesse Y, Pando RH, Sundqvist T, and Britton S. Local production of nitric oxide in patients with tuberculosis. Int $J$ Tuberc Lung Dis. 2004;8(9):1134-7.

172. Boon C, and Dick T. How Mycobacterium tuberculosis goes to sleep: the dormancy survival regulator DosR a decade later. Future Microbiol. 2012;7(4):513-8.

173. Rohde KH, Abramovitch RB, and Russell DG. Mycobacterium tuberculosis invasion of macrophages: linking bacterial gene expression to environmental cues. Cell Host Microbe. 2007;2(5):352-64.

174. Rohde KH, Veiga DF, Caldwell S, Balazsi G, and Russell DG. Linking the transcriptional profiles and the physiological states of Mycobacterium tuberculosis during an extended intracellular infection. PLoS Pathog. 2012;8(6):e1002769.

175. Wayne LG, and Hayes LG. An in vitro model for sequential study of shiftdown of Mycobacterium tuberculosis through two stages of nonreplicating persistence. Infect Immun. 1996;64(6):2062-9.

176. Bartek IL, Rutherford R, Gruppo V, Morton RA, Morris RP, Klein MR, Visconti KC, Ryan GJ, Schoolnik GK, Lenaerts A, et al. The DosR regulon of M. tuberculosis and antibacterial tolerance. Tuberculosis (Edinb). 2009;89(4):310-6.

177. McKinney JD, Honer zu Bentrup K, Munoz-Elias EJ, Miczak A, Chen B, Chan WT, Swenson D, Sacchettini JC, Jacobs WR, Jr., and Russell DG. Persistence of Mycobacterium tuberculosis in macrophages and mice requires the glyoxylate shunt enzyme isocitrate lyase. Nature. 2000;406(6797):735-8.

178. Sirakova TD, Dubey VS, Deb C, Daniel J, Korotkova TA, Abomoelak B, and Kolattukudy PE. Identification of a diacylglycerol acyltransferase gene involved in accumulation of triacylglycerol in Mycobacterium tuberculosis under stress. Microbiology. 2006;152(Pt 9):2717-25.

179. Seiler P, Ulrichs T, Bandermann S, Pradl L, Jorg S, Krenn V, Morawietz L, Kaufmann $\mathrm{SH}$, and Aichele P. Cell-wall alterations as an attribute of Mycobacterium tuberculosis in latent infection. J Infect Dis. 2003;188(9):1326-31. 
180. Leyten EM, Lin MY, Franken KL, Friggen AH, Prins C, van Meijgaarden KE, Voskuil MI, Weldingh K, Andersen P, Schoolnik GK, et al. Human T-cell responses to 25 novel antigens encoded by genes of the dormancy regulon of Mycobacterium tuberculosis. Microbes Infect. 2006;8(8):2052-60.

181. Leistikow RL, Morton RA, Bartek IL, Frimpong I, Wagner K, and Voskuil MI. The Mycobacterium tuberculosis DosR regulon assists in metabolic homeostasis and enables rapid recovery from nonrespiring dormancy. J Bacteriol. 2010;192(6):166270.

182. Epstein SS. Microbial awakenings. Nature. 2009;457(7233):1083.

183. Mariotti S, Pardini M, Gagliardi MC, Teloni R, Giannoni F, Fraziano M, Lozupone F, Meschini S, and Nisini R. Dormant Mycobacterium tuberculosis Fails To Block Phagosome Maturation and Shows Unexpected Capacity To Stimulate Specific Human T Lymphocytes. J Immunol. 2013;191(1):274-82.

184. Smith J, Manoranjan J, Pan M, Bohsali A, Xu J, Liu J, McDonald KL, Szyk A, LaRonde-LeBlanc N, and Gao LY. Evidence for pore formation in host cell membranes by ESX-1-secreted ESAT-6 and its role in Mycobacterium marinum escape from the vacuole. Infect Immun. 2008;76(12):5478-87.

185. Stamm LM, Morisaki JH, Gao LY, Jeng RL, McDonald KL, Roth R, Takeshita S, Heuser J, Welch MD, and Brown EJ. Mycobacterium marinum escapes from phagosomes and is propelled by actin-based motility. J Exp Med. 2003;198(9):1361-8.

186. de Jonge MI, Pehau-Arnaudet G, Fretz MM, Romain F, Bottai D, Brodin P, Honore N, Marchal G, Jiskoot W, England P, et al. ESAT-6 from Mycobacterium tuberculosis dissociates from its putative chaperone CFP-10 under acidic conditions and exhibits membrane-lysing activity. J Bacteriol. 2007;189(16):6028-34.

187. van der Wel N, Hava D, Houben D, Fluitsma D, van Zon M, Pierson J, Brenner M, and Peters PJ. M. tuberculosis and M. leprae translocate from the phagolysosome to the cytosol in myeloid cells. Cell. 2007;129(7):1287-98.

188. de Chastellier C. The many niches and strategies used by pathogenic mycobacteria for survival within host macrophages. Immunobiology. 2009;214(7):526-42.

189. Fortune SM, and Rubin EJ. The complex relationship between mycobacteria and macrophages: it's not all bliss. Cell Host Microbe. 2007;2(1):5-6.

190. Welin A, and Lerm M. Inside or outside the phagosome? The controversy of the intracellular localization of Mycobacterium tuberculosis. Tuberculosis (Edinb). 2012;92(2):113-20.

191. Simeone R, Bobard A, Lippmann J, Bitter W, Majlessi L, Brosch R, and Enninga J. Phagosomal rupture by Mycobacterium tuberculosis results in toxicity and host cell death. PLoS Pathog. 2012;8(2):e1002507.

192. Deretic V. Autophagy as an innate immunity paradigm: expanding the scope and repertoire of pattern recognition receptors. Curr Opin Immunol. 2012;24(1):21-31.

193. Liu G, Bi Y, Wang R, and Wang X. Self-eating and self-defense: autophagy controls innate immunity and adaptive immunity. J Leukoc Biol. 2013;93(4):511-9.

194. Russell RC, Tian Y, Yuan H, Park HW, Chang YY, Kim J, Kim H, Neufeld TP, Dillin A, and Guan KL. ULK1 induces autophagy by phosphorylating Beclin-1 and activating VPS34 lipid kinase. Nat Cell Biol. 2013;15(7):741-50.

195. Choi AM, Ryter SW, and Levine B. Autophagy in human health and disease. $N$ Engl $J$ Med. 2013;368(7):651-62.

196. Deretic V. Autophagy in immunity and cell-autonomous defense against intracellular microbes. Immunol Rev. 2011;240(1):92-104. 
197. Zheng YT, Shahnazari S, Brech A, Lamark T, Johansen T, and Brumell JH. The adaptor protein p62/SQSTM1 targets invading bacteria to the autophagy pathway. $J$ Immunol. 2009;183(9):5909-16.

198. Sanjuan MA, Dillon CP, Tait SW, Moshiach S, Dorsey F, Connell S, Komatsu M, Tanaka K, Cleveland JL, Withoff S, et al. Toll-like receptor signalling in macrophages links the autophagy pathway to phagocytosis. Nature. 2007;450(7173):1253-7.

199. Hyttinen JM, Niittykoski M, Salminen A, and Kaarniranta K. Maturation of autophagosomes and endosomes: a key role for Rab7. Biochim Biophys Acta. 2013;1833(3):503-10.

200. Yuk JM, Shin DM, Lee HM, Yang CS, Jin HS, Kim KK, Lee ZW, Lee SH, Kim JM, and Jo EK. Vitamin D3 induces autophagy in human monocytes/macrophages via cathelicidin. Cell Host Microbe. 2009;6(3):231-43.

201. Harris J, De Haro SA, Master SS, Keane J, Roberts EA, Delgado M, and Deretic V. T helper 2 cytokines inhibit autophagic control of intracellular Mycobacterium tuberculosis. Immunity. 2007;27(3):505-17.

202. Kim JJ, Lee HM, Shin DM, Kim W, Yuk JM, Jin HS, Lee SH, Cha GH, Kim JM, Lee ZW, et al. Host cell autophagy activated by antibiotics is required for their effective antimycobacterial drug action. Cell Host Microbe. 2012;11(5):457-68.

203. Ponpuak M, Davis AS, Roberts EA, Delgado MA, Dinkins C, Zhao Z, Virgin HWt, Kyei GB, Johansen T, Vergne I, et al. Delivery of cytosolic components by autophagic adaptor protein p62 endows autophagosomes with unique antimicrobial properties. Immunity. 2010;32(3):329-41.

204. Castillo EF, Dekonenko A, Arko-Mensah J, Mandell MA, Dupont N, Jiang S, Delgado-Vargas M, Timmins GS, Bhattacharya D, Yang H, et al. Autophagy protects against active tuberculosis by suppressing bacterial burden and inflammation. Proc Natl Acad Sci U S A. 2012;109(46):E3168-76.

205. King KY, Lew JD, Ha NP, Lin JS, Ma X, Graviss EA, and Goodell MA. Polymorphic allele of human IRGM1 is associated with susceptibility to tuberculosis in African Americans. PLoS ONE. 2011;6(1):e16317.

206. Romagnoli A, Etna MP, Giacomini E, Pardini M, Remoli ME, Corazzari M, Falasca L, Goletti D, Gafa V, Simeone R, et al. ESX-1 dependent impairment of autophagic flux by Mycobacterium tuberculosis in human dendritic cells. Autophagy. 2012;8(9):1357-70.

207. Zhang L, Zhang H, Zhao Y, Mao F, Wu J, Bai B, Xu Z, Jiang Y, and Shi C. Effects of Mycobacterium tuberculosis ESAT-6/CFP-10 fusion protein on the autophagy function of mouse macrophages. DNA Cell Biol. 2012;31(2):171-9.

208. de Chastellier C, Forquet F, Gordon A, and Thilo L. Mycobacterium requires an allaround closely apposing phagosome membrane to maintain the maturation block and this apposition is re-established when it rescues itself from phagolysosomes. Cell Microbiol. 2009;11(8):1190-207.

209. Saraste A, and Pulkki K. Morphologic and biochemical hallmarks of apoptosis. Cardiovasc Res. 2000;45(3):528-37.

210. Duprez L, Wirawan E, Vanden Berghe T, and Vandenabeele P. Major cell death pathways at a glance. Microbes Infect. 2009;11(13):1050-62.

211. Youle RJ, and Strasser A. The BCL-2 protein family: opposing activities that mediate cell death. Nat Rev Mol Cell Biol. 2008;9(1):47-59.

212. Li H, Zhu H, Xu CJ, and Yuan J. Cleavage of BID by caspase 8 mediates the mitochondrial damage in the Fas pathway of apoptosis. Cell. 1998;94(4):491-501. 
213. Kaczmarek A, Vandenabeele P, and Krysko DV. Necroptosis: the release of damageassociated molecular patterns and its physiological relevance. Immunity. 2013;38(2):209-23.

214. Vanlangenakker N, Vanden Berghe T, and Vandenabeele P. Many stimuli pull the necrotic trigger, an overview. Cell Death Differ. 2012;19(1):75-86.

215. Keane J, Balcewicz-Sablinska MK, Remold HG, Chupp GL, Meek BB, Fenton MJ, and Kornfeld H. Infection by Mycobacterium tuberculosis promotes human alveolar macrophage apoptosis. Infect Immun. 1997;65(1):298-304.

216. Placido R, Mancino G, Amendola A, Mariani F, Vendetti S, Piacentini M, Sanduzzi A, Bocchino ML, Zembala $\mathrm{M}$, and Colizzi V. Apoptosis of human monocytes/macrophages in Mycobacterium tuberculosis infection. $J$ Pathol. 1997;181(1):31-8.

217. Lee J, Remold HG, Ieong MH, and Kornfeld H. Macrophage apoptosis in response to high intracellular burden of Mycobacterium tuberculosis is mediated by a novel caspase-independent pathway. J Immunol. 2006;176(7):4267-74.

218. O'Sullivan MP, O'Leary S, Kelly DM, and Keane J. A caspase-independent pathway mediates macrophage cell death in response to Mycobacterium tuberculosis infection. Infect Immun. 2007;75(4):1984-93.

219. Keane J, Remold HG, and Kornfeld H. Virulent Mycobacterium tuberculosis strains evade apoptosis of infected alveolar macrophages. J Immunol. 2000;164(4):2016-20.

220. Molloy A, Laochumroonvorapong P, and Kaplan G. Apoptosis, but not necrosis, of infected monocytes is coupled with killing of intracellular bacillus Calmette-Guerin. $J$ Exp Med. 1994;180(4):1499-509.

221. Oddo M, Renno T, Attinger A, Bakker T, MacDonald HR, and Meylan PR. Fas ligand-induced apoptosis of infected human macrophages reduces the viability of intracellular Mycobacterium tuberculosis. J Immunol. 1998;160(11):5448-54.

222. Maertzdorf J, Repsilber D, Parida SK, Stanley K, Roberts T, Black G, Walzl G, and Kaufmann SH. Human gene expression profiles of susceptibility and resistance in tuberculosis. Genes Immun. 2011;12(1):15-22.

223. Edwards KM, Cynamon MH, Voladri RK, Hager CC, DeStefano MS, Tham KT, Lakey DL, Bochan MR, and Kernodle DS. Iron-cofactored superoxide dismutase inhibits host responses to Mycobacterium tuberculosis. Am J Respir Crit Care Med. 2001;164(12):2213-9.

224. Hinchey J, Lee S, Jeon BY, Basaraba RJ, Venkataswamy MM, Chen B, Chan J, Braunstein M, Orme IM, Derrick SC, et al. Enhanced priming of adaptive immunity by a proapoptotic mutant of Mycobacterium tuberculosis. $J$ Clin Invest. 2007;117(8):2279-88.

225. Velmurugan K, Chen B, Miller JL, Azogue S, Gurses S, Hsu T, Glickman M, Jacobs WR, Jr., Porcelli SA, and Briken V. Mycobacterium tuberculosis nuoG is a virulence gene that inhibits apoptosis of infected host cells. PLoS Pathog. 2007;3(7):e110.

226. Gan H, Lee J, Ren F, Chen M, Kornfeld H, and Remold HG. Mycobacterium tuberculosis blocks crosslinking of annexin-1 and apoptotic envelope formation on infected macrophages to maintain virulence. Nat Immunol. 2008;9(10):1189-97.

227. Chen M, Divangahi M, Gan H, Shin DS, Hong S, Lee DM, Serhan CN, Behar SM, and Remold HG. Lipid mediators in innate immunity against tuberculosis: opposing roles of PGE2 and LXA4 in the induction of macrophage death. J Exp Med. 2008;205(12):2791-801.

228. Bafica A, Scanga CA, Serhan C, Machado F, White S, Sher A, and Aliberti J. Host control of Mycobacterium tuberculosis is regulated by 5-lipoxygenase-dependent lipoxin production. J Clin Invest. 2005;115(6):1601-6. 
229. De Leon J, Jiang G, Ma Y, Rubin E, Fortune S, and Sun J. Mycobacterium tuberculosis ESAT-6 exhibits a unique membrane-interacting activity that is not found in its ortholog from non-pathogenic Mycobacterium smegmatis. $J$ Biol Chem. 2012;287(53):44184-91.

230. Martin CJ, Booty MG, Rosebrock TR, Nunes-Alves C, Desjardins DM, Keren I, Fortune SM, Remold HG, and Behar SM. Efferocytosis is an innate antibacterial mechanism. Cell Host Microbe. 2012;12(3):289-300.

231. Lowe DM, Redford PS, Wilkinson RJ, O'Garra A, and Martineau AR. Neutrophils in tuberculosis: friend or foe? Trends Immunol. 2012;33(1):14-25.

232. Persson A, Blomgran-Julinder R, Eklund D, Lundstrom C, and Stendahl O. Induction of apoptosis in human neutrophils by Mycobacterium tuberculosis is dependent on mature bacterial lipoproteins. Microb Pathogenesis. 2009;47(3):143-50.

233. Lopez M, Sly LM, Luu Y, Young D, Cooper H, and Reiner NE. The 19-kDa Mycobacterium tuberculosis protein induces macrophage apoptosis through toll-like receptor-2. Journal of Immunology. 2003;170(5):2409-16.

234. Erwig LP, and Henson PM. Clearance of apoptotic cells by phagocytes. Cell Death and Differentiation. 2008;15(2):243-50.

235. Krysko DV, D'Herde K, and Vandenabeele P. Clearance of apoptotic and necrotic cells and its immunological consequences. Apoptosis. 2006;11(10):1709-26.

236. Persson YAZ, Blomgran-Julinder R, Rahman S, Zheng LM, and Stendahl O. Mycobacterium tuberculosis-induced apoptotic neutrophils trigger a pro-inflammatory response in macrophages through release of heat shock protein 72, acting in synergy with the bacteria. Microbes and Infection. 2008;10(3):233-40.

237. Lucas M, Stuart LM, Savill J, and Lacy-Hulbert A. Apoptotic cells and innate immune stimuli combine to regulate macrophage cytokine secretion. Journal of Immunology. 2003;171(5):2610-5.

238. Yang CT, Cambier CJ, Davis JM, Hall CJ, Crosier PS, and Ramakrishnan L. Neutrophils exert protection in the early tuberculous granuloma by oxidative killing of mycobacteria phagocytosed from infected macrophages. Cell Host Microbe. 2012;12(3):301-12.

239. Hartman ML, and Kornfeld H. Interactions between Naive and Infected Macrophages Reduce Mycobacterium tuberculosis Viability. PLoS ONE. 2011;6(11).

240. Behar SM. Antigen-specific CD8(+) T cells and protective immunity to tuberculosis. Adv Exp Med Biol. 2013;783(141-63.

241. Noss EH, Pai RK, Sellati TJ, Radolf JD, Belisle J, Golenbock DT, Boom WH, and Harding CV. Toll-like receptor 2-dependent inhibition of macrophage class II MHC expression and antigen processing by $19-\mathrm{kDa}$ lipoprotein of Mycobacterium tuberculosis. J Immunol. 2001;167(2):910-8.

242. Pai RK, Pennini ME, Tobian AA, Canaday DH, Boom WH, and Harding CV. Prolonged toll-like receptor signaling by Mycobacterium tuberculosis and its 19kilodalton lipoprotein inhibits gamma interferon-induced regulation of selected genes in macrophages. Infect Immun. 2004;72(11):6603-14.

243. Pennini ME, Liu Y, Yang J, Croniger CM, Boom WH, and Harding CV. CCAAT/enhancer-binding protein beta and delta binding to CIITA promoters is associated with the inhibition of CIITA expression in response to Mycobacterium tuberculosis 19-kDa lipoprotein. J Immunol. 2007;179(10):6910-8.

244. Repique CJ, Li A, Brickey WJ, Ting JP, Collins FM, and Morris SL. Susceptibility of mice deficient in the MHC class II transactivator to infection with Mycobacterium tuberculosis. Scand J Immunol. 2003;58(1):15-22. 
245. Roura-Mir C, Wang L, Cheng TY, Matsunaga I, Dascher CC, Peng SL, Fenton MJ, Kirschning C, and Moody DB. Mycobacterium tuberculosis regulates CD1 antigen presentation pathways through TLR-2. J Immunol. 2005;175(3):1758-66.

246. Stenger S, Niazi KR, and Modlin RL. Down-regulation of CD1 on antigen-presenting cells by infection with Mycobacterium tuberculosis. J Immunol. 1998;161(7):3582-8.

247. Behar SM, Dascher CC, Grusby MJ, Wang CR, and Brenner MB. Susceptibility of mice deficient in CD1D or TAP1 to infection with Mycobacterium tuberculosis. J Exp Med. 1999;189(12):1973-80.

248. Harriff MJ, Purdy GE, and Lewinsohn DM. Escape from the Phagosome: The Explanation for MHC-I Processing of Mycobacterial Antigens? Front Immunol. 2012;3(40.

249. Schaible UE, Winau F, Sieling PA, Fischer K, Collins HL, Hagens K, Modlin RL, Brinkmann V, and Kaufmann SH. Apoptosis facilitates antigen presentation to $\mathrm{T}$ lymphocytes through MHC-I and CD1 in tuberculosis. Nat Med. 2003;9(8):1039-46.

250. Ramachandra L, Noss E, Boom WH, and Harding CV. Processing of Mycobacterium tuberculosis antigen 85B involves intraphagosomal formation of peptide-major histocompatibility complex II complexes and is inhibited by live bacilli that decrease phagosome maturation. J Exp Med. 2001;194(10):1421-32.

251. Blomgran R, Desvignes L, Briken V, and Ernst JD. Mycobacterium tuberculosis inhibits neutrophil apoptosis, leading to delayed activation of naive CD4 T cells. Cell Host Microbe. 2012;11(1):81-90.

252. Blomgran R, and Ernst JD. Lung neutrophils facilitate activation of naive antigenspecific CD4+ $\mathrm{T}$ cells during Mycobacterium tuberculosis infection. J Immunol. 2011;186(12):7110-9.

253. Franchi L, Amer A, Body-Malapel M, Kanneganti TD, Ozoren N, Jagirdar R, Inohara $\mathrm{N}$, Vandenabeele P, Bertin J, Coyle A, et al. Cytosolic flagellin requires Ipaf for activation of caspase-1 and interleukin 1beta in salmonella-infected macrophages. Nat Immunol. 2006;7(6):576-82.

254. Hornung V, Ablasser A, Charrel-Dennis M, Bauernfeind F, Horvath G, Caffrey DR, Latz E, and Fitzgerald KA. AIM2 recognizes cytosolic dsDNA and forms a caspase-1activating inflammasome with ASC. Nature. 2009;458(7237):514-8.

255. Martinon F, Mayor A, and Tschopp J. The inflammasomes: guardians of the body. Annu Rev Immunol. 2009;27(229-65.

256. Goto M, Katayama KI, Shirakawa F, and Tanaka I. Involvement of NF-kappaB p50/p65 heterodimer in activation of the human pro-interleukin-1beta gene at two subregions of the upstream enhancer element. Cytokine. 1999;11(1):16-28.

257. Bauernfeind FG, Horvath G, Stutz A, Alnemri ES, MacDonald K, Speert D, Fernandes-Alnemri T, Wu J, Monks BG, Fitzgerald KA, et al. Cutting edge: NFkappaB activating pattern recognition and cytokine receptors license NLRP3 inflammasome activation by regulating NLRP3 expression. $J$ Immunol. 2009;183(2):787-91.

258. Kahlenberg JM, and Dubyak GR. Mechanisms of caspase-1 activation by P2X7 receptor-mediated K+ release. Am J Physiol Cell Physiol. 2004;286(5):C1100-8.

259. Petrilli V, Papin S, Dostert C, Mayor A, Martinon F, and Tschopp J. Activation of the NALP3 inflammasome is triggered by low intracellular potassium concentration. Cell Death Differ. 2007;14(9):1583-9.

260. Munoz-Planillo R, Kuffa P, Martinez-Colon G, Smith BL, Rajendiran TM, and Nunez G. K(+) Efflux Is the Common Trigger of NLRP3 Inflammasome Activation by Bacterial Toxins and Particulate Matter. Immunity. 2013;38(6):1142-53. 
261. Kanneganti TD, Lamkanfi M, Kim YG, Chen G, Park JH, Franchi L, Vandenabeele P, and Nunez G. Pannexin-1-mediated recognition of bacterial molecules activates the cryopyrin inflammasome independent of Toll-like receptor signaling. Immunity. 2007;26(4):433-43.

262. Marina-Garcia N, Franchi L, Kim YG, Miller D, McDonald C, Boons GJ, and Nunez G. Pannexin-1-mediated intracellular delivery of muramyl dipeptide induces caspase-1 activation via cryopyrin/NLRP3 independently of Nod2. $J$ Immunol. 2008;180(6):4050-7.

263. Qu Y, Misaghi S, Newton K, Gilmour LL, Louie S, Cupp JE, Dubyak GR, Hackos D, and Dixit VM. Pannexin-1 is required for ATP release during apoptosis but not for inflammasome activation. J Immunol. 2011;186(11):6553-61.

264. Schroder K, Zhou R, and Tschopp J. The NLRP3 inflammasome: a sensor for metabolic danger? Science. 2010;327(5963):296-300.

265. Dostert C, Petrilli V, Van Bruggen R, Steele C, Mossman BT, and Tschopp J. Innate immune activation through Nalp3 inflammasome sensing of asbestos and silica. Science. 2008;320(5876):674-7.

266. Hornung V, Bauernfeind F, Halle A, Samstad EO, Kono H, Rock KL, Fitzgerald KA, and Latz E. Silica crystals and aluminum salts activate the NALP3 inflammasome through phagosomal destabilization. Nat Immunol. 2008;9(8):847-56.

267. Martinon F. Pétrilli V. Mayor A. Tardivel A. Tschopp J. Gout-associated Uric Acid Crystals Activate the NALP3 Inflammasome. Nature. 2006;440(7081):237-41.

268. Davis MJ, and Swanson JA. Technical advance: Caspase-1 activation and IL-1beta release correlate with the degree of lysosome damage, as illustrated by a novel imaging method to quantify phagolysosome damage. J Leukoc Biol. 2010;88(4):81322.

269. Duncan JA, Gao X, Huang MT, O'Connor BP, Thomas CE, Willingham SB, Bergstralh DT, Jarvis GA, Sparling PF, and Ting JP. Neisseria gonorrhoeae activates the proteinase cathepsin B to mediate the signaling activities of the NLRP3 and ASCcontaining inflammasome. J Immunol. 2009;182(10):6460-9.

270. Willingham SB, Allen IC, Bergstralh DT, Brickey WJ, Huang MT, Taxman DJ, Duncan JA, and Ting JP. NLRP3 (NALP3, Cryopyrin) facilitates in vivo caspase-1 activation, necrosis, and HMGB1 release via inflammasome-dependent and independent pathways. J Immunol. 2009;183(3):2008-15.

271. Gross O, Poeck H, Bscheider M, Dostert C, Hannesschlager N, Endres S, Hartmann G, Tardivel A, Schweighoffer E, Tybulewicz V, et al. Syk kinase signalling couples to the Nlrp3 inflammasome for anti-fungal host defence. Nature. 2009;459(7245):433-6.

272. Cruz CM, Rinna A, Forman HJ, Ventura AL, Persechini PM, and Ojcius DM. ATP activates a reactive oxygen species-dependent oxidative stress response and secretion of proinflammatory cytokines in macrophages. J Biol Chem. 2007;282(5):2871-9.

273. Pétrilli V. Papin S. Dostert C. Mayor A. Martinon F. Tschopp J. Activation of the NALP3 Inflammasome is Triggered by Low Intracellular Potassium Concentration. Cell Death and Diffrentiation. 2007;14(9):1583-9.

274. van Bruggen R, Koker MY, Jansen M, van Houdt M, Roos D, Kuijpers TW, and van den Berg TK. Human NLRP3 inflammasome activation is Nox1-4 independent. Blood. 2010;115(26):5398-400.

275. Facundo HT, de Paula JG, and Kowaltowski AJ. Mitochondrial ATP-sensitive K+ channels are redox-sensitive pathways that control reactive oxygen species production. Free Radic Biol Med. 2007;42(7):1039-48.

276. Zhou R, Yazdi AS, Menu P, and Tschopp J. A role for mitochondria in NLRP3 inflammasome activation. Nature. 2011;469(7329):221-5. 
277. Nakahira K, Haspel JA, Rathinam VA, Lee SJ, Dolinay T, Lam HC, Englert JA, Rabinovitch M, Cernadas M, Kim HP, et al. Autophagy proteins regulate innate immune responses by inhibiting the release of mitochondrial DNA mediated by the NALP3 inflammasome. Nat Immunol. 2011;12(3):222-30.

278. Saitoh T, Fujita N, Jang MH, Uematsu S, Yang BG, Satoh T, Omori H, Noda T, Yamamoto N, Komatsu M, et al. Loss of the autophagy protein Atg16L1 enhances endotoxin-induced IL-1beta production. Nature. 2008;456(7219):264-8.

279. Shimada K, Crother TR, Karlin J, Dagvadorj J, Chiba N, Chen S, Ramanujan VK, Wolf AJ, Vergnes L, Ojcius DM, et al. Oxidized mitochondrial DNA activates the NLRP3 inflammasome during apoptosis. Immunity. 2012;36(3):401-14.

280. Zhou R, Tardivel A, Thorens B, Choi I, and Tschopp J. Thioredoxin-interacting protein links oxidative stress to inflammasome activation. Nat Immunol. 2010;11(2):136-40.

281. Bauernfeind F, Bartok E, Rieger A, Franchi L, Nunez G, and Hornung V. Cutting edge: reactive oxygen species inhibitors block priming, but not activation, of the NLRP3 inflammasome. J Immunol. 2011;187(2):613-7.

282. Valanne S, Wang JH, and Ramet M. The Drosophila Toll signaling pathway. $J$ Immunol. 2011;186(2):649-56.

283. Carlstrom M, Ekman AK, Petersson S, Soderkvist P, and Enerback C. Genetic support for the role of the NLRP3 inflammasome in psoriasis susceptibility. Exp Dermatol. 2012;21(12):932-7.

284. Mathews RJ, Robinson JI, Battellino M, Wong C, Taylor JC, Eyre S, Churchman SM, Wilson AG, Isaacs JD, Hyrich K, et al. Evidence of NLRP3-inflammasome activation in rheumatoid arthritis (RA); genetic variants within the NLRP3-inflammasome complex in relation to susceptibility to RA and response to anti-TNF treatment. Ann Rheum Dis. 2013.

285. Qing YF, Zhang QB, and Zhou JG. Innate immunity functional gene polymorphisms and gout susceptibility. Gene. 2013;524(2):412-4.

286. Villani AC, Lemire M, Fortin G, Louis E, Silverberg MS, Collette C, Baba N, Libioulle C, Belaiche J, Bitton A, et al. Common variants in the NLRP3 region contribute to Crohn's disease susceptibility. Nat Genet. 2009;41(1):71-6.

287. Neven B, Prieur AM, and Quartier dit Maire P. Cryopyrinopathies: update on pathogenesis and treatment. Nat Clin Pract Rheumatol. 2008;4(9):481-9.

288. Touitou I, Lesage S, McDermott M, Cuisset L, Hoffman H, Dode C, Shoham N, Aganna E, Hugot JP, Wise C, et al. Infevers: an evolving mutation database for autoinflammatory syndromes. Hum Mutat. 2004;24(3):194-8.

289. Aksentijevich I, C DP, Remmers EF, Mueller JL, Le J, Kolodner RD, Moak Z, Chuang M, Austin F, Goldbach-Mansky R, et al. The clinical continuum of cryopyrinopathies: novel CIAS1 mutations in North American patients and a new cryopyrin model. Arthritis Rheum. 2007;56(4):1273-85.

290. Hoffman HM, and Brydges SD. Genetic and molecular basis of inflammasomemediated disease. J Biol Chem. 2011;286(13):10889-96.

291. Dowds TA, Masumoto J, Zhu L, Inohara N, and Nunez G. Cryopyrin-induced interleukin 1beta secretion in monocytic cells: enhanced activity of disease-associated mutants and requirement for ASC. J Biol Chem. 2004;279(21):21924-8.

292. Fiorentino DF, Zlotnik A, Mosmann TR, Howard M, and O'Garra A. IL-10 inhibits cytokine production by activated macrophages. J Immunol. 1991;147(11):3815-22.

293. Guarda G, Braun M, Staehli F, Tardivel A, Mattmann C, Forster I, Farlik M, Decker $\mathrm{T}$, Du Pasquier RA, Romero P, et al. Type I interferon inhibits interleukin-1 production and inflammasome activation. Immunity. 2011;34(2):213-23. 
294. Bauernfeind F, Rieger A, Schildberg FA, Knolle PA, Schmid-Burgk JL, and Hornung V. NLRP3 inflammasome activity is negatively controlled by miR-223. J Immunol. 2012;189(8):4175-81.

295. Harris J, Hartman M, Roche C, Zeng SG, O'Shea A, Sharp FA, Lambe EM, Creagh EM, Golenbock DT, Tschopp J, et al. Autophagy controls IL-1beta secretion by targeting pro-IL-1beta for degradation. J Biol Chem. 2011;286(11):9587-97.

296. Shi CS, Shenderov K, Huang NN, Kabat J, Abu-Asab M, Fitzgerald KA, Sher A, and Kehrl JH. Activation of autophagy by inflammatory signals limits IL-1beta production by targeting ubiquitinated inflammasomes for destruction. Nat Immunol. 2012;13(3):255-63.

297. Byrne BG, Dubuisson JF, Joshi AD, Persson JJ, and Swanson MS. Inflammasome components coordinate autophagy and pyroptosis as macrophage responses to infection. MBio. 2013;4(1):e00620-12.

298. Mishra BB, Rathinam VA, Martens GW, Martinot AJ, Kornfeld H, Fitzgerald KA, and Sassetti CM. Nitric oxide controls the immunopathology of tuberculosis by inhibiting NLRP3 inflammasome-dependent processing of IL-1beta. Nat Immunol. 2013;14(1):52-60.

299. Stehlik C, and Dorfleutner A. COPs and POPs: modulators of inflammasome activity. J Immunol. 2007;179(12):7993-8.

300. Druilhe A, Srinivasula SM, Razmara M, Ahmad M, and Alnemri ES. Regulation of IL-1beta generation by Pseudo-ICE and ICEBERG, two dominant negative caspase recruitment domain proteins. Cell Death Differ. 2001;8(6):649-57.

301. Razmara M, Srinivasula SM, Wang L, Poyet JL, Geddes BJ, DiStefano PS, Bertin J, and Alnemri ES. CARD-8 protein, a new CARD family member that regulates caspase-1 activation and apoptosis. J Biol Chem. 2002;277(16):13952-8.

302. Kastbom A, Verma D, Eriksson P, Skogh T, Wingren G, and Soderkvist P. Genetic variation in proteins of the cryopyrin inflammasome influences susceptibility and severity of rheumatoid arthritis (The Swedish TIRA project). Rheumatology. 2008;47(4):415-7.

303. Roberts RL, Topless RK, Phipps-Green AJ, Gearry RB, Barclay ML, and Merriman TR. Evidence of interaction of CARD8 rs2043211 with NALP3 rs35829419 in Crohn's disease. Genes Immun. 2010;11(4):351-6.

304. Schoultz I, Verma D, Halfvarsson J, Torkvist L, Fredrikson M, Sjoqvist U, Lordal M, Tysk C, Lerm M, Soderkvist P, et al. Combined polymorphisms in genes encoding the inflammasome components NALP3 and CARD8 confer susceptibility to Crohn's disease in Swedish men. Am J Gastroenterol. 2009;104(5):1180-8.

305. Shenoy AR, Wellington DA, Kumar P, Kassa H, Booth CJ, Cresswell P, and MacMicking JD. GBP5 promotes NLRP3 inflammasome assembly and immunity in mammals. Science. 2012;336(6080):481-5.

306. Netea MG, Nold-Petry CA, Nold MF, Joosten LA, Opitz B, van der Meer JH, van de Veerdonk FL, Ferwerda G, Heinhuis B, Devesa I, et al. Differential requirement for the activation of the inflammasome for processing and release of IL-1beta in monocytes and macrophages. Blood. 2009;113(10):2324-35.

307. Abdalla H, Srinivasan L, Shah S, Mayer-Barber KD, Sher A, Sutterwala FS, and Briken V. Mycobacterium tuberculosis infection of dendritic cells leads to partially caspase-1/11-independent IL-1beta and IL-18 secretion but not to pyroptosis. PLoS ONE. 2012;7(7):e40722.

308. Dinarello CA. Immunological and inflammatory functions of the interleukin-1 family. Annu Rev Immunol. 2009;27(519-50. 
309. Vince JE, Wong WW, Gentle I, Lawlor KE, Allam R, O'Reilly L, Mason K, Gross O, Ma S, Guarda G, et al. Inhibitor of apoptosis proteins limit RIP3 kinase-dependent interleukin-1 activation. Immunity. 2012;36(2):215-27.

310. Maelfait J, Vercammen E, Janssens S, Schotte P, Haegman M, Magez S, and Beyaert R. Stimulation of Toll-like receptor 3 and 4 induces interleukin-1beta maturation by caspase-8. J Exp Med. 2008;205(9):1967-73.

311. Ting JP, Willingham SB, and Bergstralh DT. NLRs at the intersection of cell death and immunity. Nat Rev Immunol. 2008;8(5):372-9.

312. Bergsbaken T, Fink SL, and Cookson BT. Pyroptosis: host cell death and inflammation. Nat Rev Microbiol. 2009;7(2):99-109.

313. Nystrom S, Antoine DJ, Lundback P, Lock JG, Nita AF, Hogstrand K, Grandien A, Erlandsson-Harris H, Andersson U, and Applequist SE. TLR activation regulates damage-associated molecular pattern isoforms released during pyroptosis. EMBO J. 2013;32(1):86-99.

314. Fernandes-Alnemri T, Yu JW, Datta P, Wu J, and Alnemri ES. AIM2 activates the inflammasome and cell death in response to cytoplasmic DNA. Nature. 2009;458(7237):509-13.

315. Sauer JD, Witte CE, Zemansky J, Hanson B, Lauer P, and Portnoy DA. Listeria monocytogenes triggers AIM2-mediated pyroptosis upon infrequent bacteriolysis in the macrophage cytosol. Cell Host Microbe. 2010;7(5):412-9.

316. Kayagaki N, Warming S, Lamkanfi M, Vande Walle L, Louie S, Dong J, Newton K, Qu Y, Liu J, Heldens S, et al. Non-canonical inflammasome activation targets caspase-11. Nature. 2011;479(7371):117-21.

317. Brennan MA, and Cookson BT. Salmonella induces macrophage death by caspase-1dependent necrosis. Mol Microbiol. 2000;38(1):31-40.

318. Case CL, Shin S, and Roy CR. Asc and Ipaf Inflammasomes direct distinct pathways for caspase-1 activation in response to Legionella pneumophila. Infect Immun. 2009;77(5):1981-91.

319. Fink SL, Bergsbaken T, and Cookson BT. Anthrax lethal toxin and Salmonella elicit the common cell death pathway of caspase-1-dependent pyroptosis via distinct mechanisms. Proc Natl Acad Sci U S A. 2008;105(11):4312-7.

320. Fink SL, and Cookson BT. Caspase-1-dependent pore formation during pyroptosis leads to osmotic lysis of infected host macrophages. Cell Microbiol. 2006;8(11):181225.

321. Chavarria-Smith J, and Vance RE. Direct proteolytic cleavage of NLRP1B is necessary and sufficient for inflammasome activation by anthrax lethal factor. PLoS Pathog. 2013;9(6):e1003452.

322. Ceballos-Olvera I, Sahoo M, Miller MA, Del Barrio L, and Re F. Inflammasomedependent pyroptosis and IL-18 protect against Burkholderia pseudomallei lung infection while IL-1beta is deleterious. PLoS Pathog. 2011;7(12):e1002452.

323. Fernandes-Alnemri T, Wu J, Yu JW, Datta P, Miller B, Jankowski W, Rosenberg S, Zhang J, and Alnemri ES. The pyroptosome: a supramolecular assembly of ASC dimers mediating inflammatory cell death via caspase-1 activation. Cell Death Differ. 2007;14(9):1590-604.

324. Broz P, von Moltke J, Jones JW, Vance RE, and Monack DM. Differential Requirement for Caspase-1 Autoproteolysis in Pathogen-Induced Cell Death and Cytokine Processing. Cell Host \& Microbe. 2010;8(6):471-83.

325. Mariathasan S, Weiss DS, Dixit VM, and Monack DM. Innate immunity against Francisella tularensis is dependent on the ASC/caspase-1 axis. J Exp Med. 2005;202(8):1043-9. 
326. Sarkar A, Hall MW, Exline M, Hart J, Knatz N, Gatson NT, and Wewers MD. Caspase-1 regulates Escherichia coli sepsis and splenic B cell apoptosis independently of interleukin-1beta and interleukin-18. Am $J$ Respir Crit Care Med. 2006;174(9):1003-10.

327. Miao EA, Leaf IA, Treuting PM, Mao DP, Dors M, Sarkar A, Warren SE, Wewers MD, and Aderem A. Caspase-1-induced pyroptosis is an innate immune effector mechanism against intracellular bacteria. Nat Immunol. 2010;11(12):1136-42.

328. Bergsbaken T, Fink SL, den Hartigh AB, Loomis WP, and Cookson BT. Coordinated host responses during pyroptosis: caspase-1-dependent lysosome exocytosis and inflammatory cytokine maturation. J Immunol. 2011;187(5):2748-54.

329. Wree A, Eguchi A, McGeough MD, Pena CA, Johnson CD, Canbay A, Hoffman HM, and Feldstein AE. NLRP3 inflammasome activation results in hepatocyte pyroptosis, liver inflammation and fibrosis. Hepatology. 2013.

330. Brough D, and Rothwell NJ. Caspase-1-dependent processing of pro-interleukin-1beta is cytosolic and precedes cell death. J Cell Sci. 2007;120(Pt 5):772-81.

331. Le Feuvre RA, Brough D, Iwakura Y, Takeda K, and Rothwell NJ. Priming of macrophages with lipopolysaccharide potentiates P2X7-mediated cell death via a caspase-1-dependent mechanism, independently of cytokine production. J Biol Chem. 2002;277(5):3210-8.

332. Willingham SB, Bergstralh DT, O'Connor W, Morrison AC, Taxman DJ, Duncan JA, Barnoy S, Venkatesan MM, Flavell RA, Deshmukh M, et al. Microbial pathogeninduced necrotic cell death mediated by the inflammasome components CIAS1/cryopyrin/NLRP3 and ASC. Cell Host Microbe. 2007;2(3):147-59.

333. Fujisawa A, Kambe N, Saito M, Nishikomori R, Tanizaki H, Kanazawa N, Adachi S, Heike T, Sagara J, Suda T, et al. Disease-associated mutations in CIAS1 induce cathepsin B-dependent rapid cell death of human THP-1 monocytic cells. Blood. 2007;109(7):2903-11.

334. Satoh T, Kambe N, and Matsue H. NLRP3 activation induces ASC-dependent programmed necrotic cell death, which leads to neutrophilic inflammation. Cell Death Dis. 2013;4(e644.

335. Mihalik R, Imre G, Petak I, Szende B, and Kopper L. Cathepsin B-independent abrogation of cell death by CA-074-OMe upstream of lysosomal breakdown. Cell Death Differ. 2004;11(12):1357-60.

336. Shao W, Yeretssian G, Doiron K, Hussain SN, and Saleh M. The caspase-1 digestome identifies the glycolysis pathway as a target during infection and septic shock. $J$ Biol Chem. 2007;282(50):36321-9.

337. Fettelschoss A, Kistowska M, LeibundGut-Landmann S, Beer HD, Johansen P, Senti G, Contassot E, Bachmann MF, French LE, Oxenius A, et al. Inflammasome activation and IL-1beta target IL-1alpha for secretion as opposed to surface expression. Proc Natl Acad Sci U S A. 2011;108(44):18055-60.

338. Keller M, Ruegg A, Werner S, and Beer HD. Active caspase-1 is a regulator of unconventional protein secretion. Cell. 2008;132(5):818-31.

339. Sagulenko V, Thygesen SJ, Sester DP, Idris A, Cridland JA, Vajjhala PR, Roberts TL, Schroder K, Vince JE, Hill JM, et al. AIM2 and NLRP3 inflammasomes activate both apoptotic and pyroptotic death pathways via ASC. Cell Death Differ. 2013;20(9):1149-60.

340. Lamkanfi M, Kanneganti TD, Van Damme P, Vanden Berghe T, Vanoverberghe I, Vandekerckhove J, Vandenabeele P, Gevaert K, and Nunez G. Targeted peptidecentric proteomics reveals caspase-7 as a substrate of the caspase- 1 inflammasomes. Mol Cell Proteomics. 2008;7(12):2350-63. 
341. Gurcel L. Abrami L. Girardin S. Tschopp J. van der Goot FG. Caspase-1 Activation of Lipid Metabolic Pathways in Response to Bacterial Pore-forming toxins Promotes Cell Survival. Cell. 2006;126(6):1135-45.

342. Smith DE. The biological paths of IL-1 family members IL-18 and IL-33. J Leukoc Biol. 2011;89(3):383-92.

343. Cayrol C, and Girard JP. The IL-1-like cytokine IL-33 is inactivated after maturation by caspase-1. Proc Natl Acad Sci U S A. 2009;106(22):9021-6.

344. Netea MG, Kullberg BJ, Verschueren I, and Van Der Meer JW. Interleukin-18 induces production of proinflammatory cytokines in mice: no intermediate role for the cytokines of the tumor necrosis factor family and interleukin-1beta. Eur J Immunol. 2000;30(10):3057-60.

345. Dinarello CA. Biologic basis for interleukin-1 in disease. Blood. 1996;87(6):2095-147.

346. Andrei C, Margiocco P, Poggi A, Lotti LV, Torrisi MR, and Rubartelli A. Phospholipases C and A2 control lysosome-mediated IL-1 beta secretion: Implications for inflammatory processes. Proc Natl Acad Sci U S A. 2004;101(26):9745-50.

347. Janson RW, Hance KR, and Arend WP. Production of IL-1 receptor antagonist by human in vitro-derived macrophages. Effects of lipopolysaccharide and granulocytemacrophage colony-stimulating factor. J Immunol. 1991;147(12):4218-23.

348. Arend WP. The balance between IL-1 and IL-1Ra in disease. Cytokine Growth Factor Rev. 2002;13(4-5):323-40.

349. Cullinan EB, Kwee L, Nunes P, Shuster DJ, Ju G, McIntyre KW, Chizzonite RA, and Labow MA. IL-1 receptor accessory protein is an essential component of the IL-1 receptor. J Immunol. 1998;161(10):5614-20.

350. Colotta F, Re F, Muzio M, Bertini R, Polentarutti N, Sironi M, Giri JG, Dower SK, Sims JE, and Mantovani A. Interleukin-1 type II receptor: a decoy target for IL-1 that is regulated by IL-4. Science. 1993;261(5120):472-5.

351. Dinarello CA. Interleukin-1 in the pathogenesis and treatment of inflammatory diseases. Blood. 2011;117(14):3720-32.

352. Lang D, Knop J, Wesche H, Raffetseder U, Kurrle R, Boraschi D, and Martin MU. The type II IL-1 receptor interacts with the IL-1 receptor accessory protein: a novel mechanism of regulation of IL-1 responsiveness. J Immunol. 1998;161(12):6871-7.

353. Mishra BB, Moura-Alves P, Sonawane A, Hacohen N, Griffiths G, Moita LF, and Anes E. Mycobacterium tuberculosis Protein ESAT-6 is a Potent Activator of the NLRP3/ASC Inflammasome. Cell Microbiol. 2010.

354. Dorhoi A, Nouailles G, Jorg S, Hagens K, Heinemann E, Pradl L, Oberbeck-Muller D, Duque-Correa MA, Reece ST, Ruland J, et al. Activation of the NLRP3 inflammasome by Mycobacterium tuberculosis is uncoupled from susceptibility to active tuberculosis. Eur J Immunol. 2012;42(2):374-84.

355. McElvania Tekippe E, Allen IC, Hulseberg PD, Sullivan JT, McCann JR, Sandor M, Braunstein M, and Ting JP. Granuloma formation and host defense in chronic Mycobacterium tuberculosis infection requires PYCARD/ASC but not NLRP3 or caspase-1. PLoS ONE. 2010;5(8):e12320.

356. Walter K, Holscher C, Tschopp J, and Ehlers S. NALP3 is not necessary for early protection against experimental tuberculosis. Immunobiology. 2010;215(9-10):804-11.

357. Liu PT, Schenk M, Walker VP, Dempsey PW, Kanchanapoomi M, Wheelwright M, Vazirnia A, Zhang X, Steinmeyer A, Zugel U, et al. Convergence of IL-1beta and VDR activation pathways in human TLR2/1-induced antimicrobial responses. PLoS ONE. 2009;4(6):e5810.

358. Verway M, Bouttier M, Wang TT, Carrier M, Calderon M, An BS, Devemy E, McIntosh F, Divangahi M, Behr MA, et al. Vitamin D induces interleukin-1beta 
expression: paracrine macrophage epithelial signaling controls $M$. tuberculosis infection. PLoS Pathog. 2013;9(6):e1003407.

359. Pilli M, Arko-Mensah J, Ponpuak M, Roberts E, Master S, Mandell MA, Dupont N, Ornatowski W, Jiang S, Bradfute SB, et al. TBK-1 promotes autophagy-mediated antimicrobial defense by controlling autophagosome maturation. Immunity. 2012;37(2):223-34.

360. Fremond CM, Yeremeev V, Nicolle DM, Jacobs M, Quesniaux VF, and Ryffel B. Fatal Mycobacterium tuberculosis infection despite adaptive immune response in the absence of MyD88. J Clin Invest. 2004;114(12):1790-9.

361. Scanga CA, Bafica A, Feng CG, Cheever AW, Hieny S, and Sher A. MyD88-deficient mice display a profound loss in resistance to Mycobacterium tuberculosis associated with partially impaired Th1 cytokine and nitric oxide synthase 2 expression. Infect Immun. 2004;72(4):2400-4.

362. Fremond CM, Togbe D, Doz E, Rose S, Vasseur V, Maillet I, Jacobs M, Ryffel B, and Quesniaux VF. IL-1 receptor-mediated signal is an essential component of MyD88dependent innate response to Mycobacterium tuberculosis infection. $J$ Immunol. 2007;179(2):1178-89.

363. Mayer-Barber KD, Barber DL, Shenderov K, White SD, Wilson MS, Cheever A, Kugler D, Hieny S, Caspar P, Nunez G, et al. Caspase-1 independent IL-1beta production is critical for host resistance to mycobacterium tuberculosis and does not require TLR signaling in vivo. $J$ Immunol. 2010;184(7):3326-30.

364. Mayer-Barber KD, Andrade BB, Barber DL, Hieny S, Feng CG, Caspar P, Oland S, Gordon S, and Sher A. Innate and adaptive interferons suppress IL-1alpha and IL1beta production by distinct pulmonary myeloid subsets during Mycobacterium tuberculosis infection. Immunity. 2011;35(6):1023-34.

365. Carlsson F, Kim J, Dumitru C, Barck KH, Carano RA, Sun M, Diehl L, and Brown EJ. Host-detrimental role of Esx-1-mediated inflammasome activation in mycobacterial infection. PLoS Pathog. 2010;6(5):e1000895.

366. Fontan P, Aris V, Ghanny S, Soteropoulos P, and Smith I. Global transcriptional profile of Mycobacterium tuberculosis during THP-1 human macrophage infection. Infect Immun. 2008;76(2):717-25.

367. Tailleux L, Waddell SJ, Pelizzola M, Mortellaro A, Withers M, Tanne A, Castagnoli PR, Gicquel B, Stoker NG, Butcher PD, et al. Probing host pathogen cross-talk by transcriptional profiling of both Mycobacterium tuberculosis and infected human dendritic cells and macrophages. PLOS ONE. 2008;3(1):e1403.

368. D'Souza S, Rosseels V, Denis O, Tanghe A, De Smet N, Jurion F, Palfliet K, Castiglioni N, Vanonckelen A, Wheeler C, et al. Improved tuberculosis DNA vaccines by formulation in cationic lipids. Infect Immun. 2002;70(7):3681-8.

369. Rosseels V, Roupie V, Zinniel D, Barletta RG, and Huygen K. Development of luminescent Mycobacterium avium subsp. paratuberculosis for rapid screening of vaccine candidates in mice. Infect Immun. 2006;74(6):3684-6.

370. Tanghe A, D'Souza S, Rosseels V, Denis O, Ottenhoff TH, Dalemans W, Wheeler C, and Huygen K. Improved immunogenicity and protective efficacy of a tuberculosis DNA vaccine encoding Ag85 by protein boosting. Infect Immun. 2001;69(5):3041-7.

371. Snewin VA, Gares MP, Gaora PO, Hasan Z, Brown IN, and Young DB. Assessment of immunity to mycobacterial infection with luciferase reporter constructs. Infect Immun. 1999;67(9):4586-93.

372. Chen M, Gan H, and Remold HG. A mechanism of virulence: virulent Mycobacterium tuberculosis strain H37Rv, but not attenuated H37Ra, causes significant mitochondrial 
inner membrane disruption in macrophages leading to necrosis. $J$ Immunol. 2006;176(6):3707-16.

373. Lee J, Repasy T, Papavinasasundaram K, Sassetti C, and Kornfeld H. Mycobacterium tuberculosis induces an atypical cell death mode to escape from infected macrophages. PLoS ONE. 2011;6(3):e18367.

374. Wong KW, and Jacobs WR, Jr. Critical role for NLRP3 in necrotic death triggered by Mycobacterium tuberculosis. Cell Microbiol. 2011;13(9):1371-84.

375. Martineau AR, Timms PM, Bothamley GH, Hanifa Y, Islam K, Claxton AP, Packe GE, Moore-Gillon JC, Darmalingam M, Davidson RN, et al. High-dose vitamin D(3) during intensive-phase antimicrobial treatment of pulmonary tuberculosis: a doubleblind randomised controlled trial. Lancet. 2011;377(9761):242-50.

376. Pakasi TA, Karyadi E, Suratih NM, Salean M, Darmawidjaja N, Bor H, van der Velden K, Dolmans WM, and van der Meer JW. Zinc and vitamin A supplementation fails to reduce sputum conversion time in severely malnourished pulmonary tuberculosis patients in Indonesia. Nutr J. 2010;9(41.

377. Schon T, Idh J, Westman A, Elias D, Abate E, Diro E, Moges F, Kassu A, Ayele B, Forslund T, et al. Effects of a food supplement rich in arginine in patients with smear positive pulmonary tuberculosis--a randomised trial. Tuberculosis (Edinb). 2011;91(5):370-7.

378. Wejse C, Gomes VF, Rabna P, Gustafson P, Aaby P, Lisse IM, Andersen PL, Glerup $\mathrm{H}$, and Sodemann M. Vitamin D as supplementary treatment for tuberculosis: a double-blind, randomized, placebo-controlled trial. Am J Respir Crit Care Med. 2009;179(9):843-50.

379. White JH. Vitamin D signaling, infectious diseases, and regulation of innate immunity. Infect Immun. 2008;76(9):3837-43.

380. Campbell GR, and Spector SA. Vitamin D inhibits human immunodeficiency virus type 1 and Mycobacterium tuberculosis infection in macrophages through the induction of autophagy. PLoS Pathog. 2012;8(5):e1002689.

381. Krutzik SR, Hewison M, Liu PT, Robles JA, Stenger S, Adams JS, and Modlin RL. IL-15 links TLR2/1-induced macrophage differentiation to the vitamin D-dependent antimicrobial pathway. J Immunol. 2008;181(10):7115-20.

382. Liu PT, Stenger S, Tang DH, and Modlin RL. Cutting edge: vitamin D-mediated human antimicrobial activity against Mycobacterium tuberculosis is dependent on the induction of cathelicidin. J Immunol. 2007;179(4):2060-3.

383. Martineau AR, Wilkinson KA, Newton SM, Floto RA, Norman AW, Skolimowska K, Davidson RN, Sorensen OE, Kampmann B, Griffiths CJ, et al. IFN-gamma- and TNFindependent vitamin D-inducible human suppression of mycobacteria: the role of cathelicidin LL-37. J Immunol. 2007;178(11):7190-8.

384. Rook GA, Steele J, Fraher L, Barker S, Karmali R, O'Riordan J, and Stanford J. Vitamin D3, gamma interferon, and control of proliferation of Mycobacterium tuberculosis by human monocytes. Immunology. 1986;57(1):159-63.

385. Lee BN, Kim TH, Jun JB, Yoo DH, Woo JH, Choi SJ, Lee YH, Song GG, Kim Y, Lee JY, et al. Upregulation of interleukin-1beta production by 1,25-dihydroxyvitamin D(3) in activated human macrophages. Mol Biol Rep. 2011;38(3):2193-201.

386. Kleinnijenhuis J, Quintin J, Preijers F, Joosten LA, Ifrim DC, Saeed S, Jacobs C, van Loenhout J, de Jong D, Stunnenberg HG, et al. Bacille Calmette-Guerin induces NOD2-dependent nonspecific protection from reinfection via epigenetic reprogramming of monocytes. Proc Natl Acad Sci U S A. 2012;109(43):17537-42. 
387. Zheng L, He M, Long M, Blomgran R, and Stendahl O. Pathogen-induced apoptotic neutrophils express heat shock proteins and elicit activation of human macrophages. $J$ Immunol. 2004;173(10):6319-26.

388. Persson YA, Blomgran-Julinder R, Rahman S, Zheng L, and Stendahl O. Mycobacterium tuberculosis-induced apoptotic neutrophils trigger a pro-inflammatory response in macrophages through release of heat shock protein 72, acting in synergy with the bacteria. Microbes Infect. 2008;10(3):233-40.

389. Braian C, Hogea V, and Stendahl O. Mycobacterium tuberculosis- Induced Neutrophil Extracellular Traps Activate Human Macrophages. J Innate Immun. 2013.

390. Fadok VA, Bratton DL, Konowal A, Freed PW, Westcott JY, and Henson PM. Macrophages that have ingested apoptotic cells in vitro inhibit proinflammatory cytokine production through autocrine/paracrine mechanisms involving TGF-beta, PGE2, and PAF. J Clin Invest. 1998;101(4):890-8.

391. Jayaraman P, Sada-Ovalle I, Nishimura T, Anderson AC, Kuchroo VK, Remold HG, and Behar SM. IL-1beta promotes antimicrobial immunity in macrophages by regulating TNFR signaling and caspase-3 activation. J Immunol. 2013;190(8):4196204.

392. Verma D, Sarndahl E, Andersson H, Eriksson P, Fredrikson M, Jonsson JI, Lerm M, and Soderkvist P. The Q705K polymorphism in NLRP3 is a gain-of-function alteration leading to excessive interleukin-1beta and IL-18 production. PLoS ONE. 2012;7(4):e34977.

393. Sahdo B, Fransén K, Asfaw Idosa B, Eriksson P, Söderquist B, Kelly A, and E S. Cytokine Profile in a Cohort of Healthy Blood Donors Carrying Polymorphisms in Genes Encoding the NLRP3 Inflammasome. PLOS ONE. 2013;In press(

394. Verma D, Lerm M, Blomgran Julinder R, Eriksson P, Soderkvist P, and Sarndahl E. Gene polymorphisms in the NALP3 inflammasome are associated with interleukin-1 production and severe inflammation: relation to common inflammatory diseases? Arthritis Rheum. 2008;58(3):888-94.

395. Verma D, Eriksson P, Sahdo B, Persson A, Ejdeback M, Sarndahl E, and Soderkvist P. Two adult siblings with atypical cryopyrin-associated periodic syndrome due to a novel M299V mutation in NLRP3. Arthritis Rheum. 2010;62(7):2138-43.

396. Fontalba A, Martinez-Taboada V, Gutierrez O, Pipaon C, Benito N, Balsa A, Blanco $\mathrm{R}$, and Fernandez-Luna JL. Deficiency of the NF-kappa B inhibitor caspase activating and, recruitment domain 8 in patients with rheumatoid arthritis is associated with disease severity. Journal of Immunology. 2007;179(7):4867-73.

397. Ko DC, Shukla KP, Fong C, Wasnick M, Brittnacher MJ, Wurfel MM, Holden TD, O'Keefe GE, Van Yserloo B, Akey JM, et al. A genome-wide in vitro bacterialinfection screen reveals human variation in the host response associated with inflammatory disease. Am J Hum Genet. 2009;85(2):214-27. 


\section{ACKNOWLEDGMNTS}

This thesis has been made possible through the contributions of many people to whom I am very grateful.

My three supervisors,

To my main supervisor Maria Lerm, whose undaunting enthusiasm and dedication to science have been a constant motivation throughout my years as a PhD student.

To my co-supervisor Olle Stendahl, for constantly reminding me to see the bigger picture in everything and for being able to always reassure me in my moments of doubt.

To my co-supervisor, Eva Särndahl, for always being very supportive, for your invaluable knowledge on all things related to NLRP3 and the inflammasome and for taking me to see Charles Dinarello talk about IL-1.

To colleagues, old and new, involved in various aspects of the tuberculosis research at Medical Microbiology. To Amanda Welin, for all the discussions and the many hours of working together. To Johanna Raffetseder, for your delightful company and your amazing organization skills. To Elsje Pienaar, for immediately becoming a part of the group with your friendly and helpful attitude. To Alexander Persson, for supervising my very first scientific project and kindling my interest in research. To Henrik and Blanka Andersson, for all the chats and your valiant efforts on the apoptotic neutrophil project. To Clara Braian, Fanny Herhold, Sadaf Kalsum, Eyler Ngoh, Parasa Venkata Ramanarao, Robert Blomgran, Veronika Patcha Brodin and Kristina Orselius for all your help in matters small and big. To Jonna Idh and Ebba Abate, for sharing your knowledge of the clinical aspects of the disease. To Lennart Persson and all the members of the FORSS group led by Thomas Schön, for a fruitful collaboration and interesting discussions. To the staff at Clinical Microbiology working in the BSL3 facility; Britt-Marie, Ann-Marie, Sara, Eva, Slavica, Michaela and Helen, for your patience and friendly manners. To Peter Söderkvist and Deepti Verma, whose work on the inflammasome has been a major source of inspiration to this thesis. To all the students, which have been a part of the group and contributed in various ways to the different projects. An honorable mention to Thommie Karlsson, who almost contributed to the work presented in this thesis, but then forgot to transfect the cells.

To all my other colleagues at Medical Microbiology who has been very instrumental in making both my years as a PhD student and the coffee breaks completely fly by. 
Thank you for all the laughs, I will surely miss all the funny discussions. I'm am very grateful for all the help I've received over the years and I'm glad I got to know all of you.

To my friends and family for so efficiently taking my mind of work. A special thanks to Lars Wikberg, who took the time from his otherwise very busy summer to read through the draft of this thesis.

To Louise, for being my loving wife, best friend and fellow PhD student all wrapped in one. 


\section{Papers}

The articles associated with this thesis have been removed for copyright reasons. For more details about these see:

http://urn.kb.se/resolve?urn=urn:nbn:se:liu:diva-100890 